\title{
On Compressible Smooth Viscous Fluids in Slowly Expanding Balls
}

\author{
Huicheng Yin* and Lin Zhang \\ School of Mathematical Sciences, Jiangsu Provincial Key Laboratory for Numerical \\ Simulation of Large Scale Complex Systems, Nanjing Normal University, \\ Nanjing 210023, Jiangsu, China
}

Received 3 January 2016; Accepted (in revised version) 15 December 2016

\begin{abstract}
In [17] and [19,20], the global existence and large time behaviors of smooth compressible fluids (including inviscid gases of Euler equations, viscous gases of Navier-Stokes equations, and rarified gases of Boltzmann equation, respectively) have been established in an infinitely expanding ball with a constant expansion speed. This paper concerns with the viscous fluids in a slowly expanding ball. By involved analysis on the density function and the weighted energy estimates, we show that the fluid in the slowly expanding ball smoothly tends to a vacuum state and there is no appearance of vacuum in any part of the expansive ball. Our present result is a meaningful supplement to the one in [19].
\end{abstract}

Key Words: Compressible Navier-Stokes equations, slowly expanding ball, weighted energy estimate, global existence.

AMS Subject Classifications: 35L70, 35L65, 35L67, 76N15

\section{Introduction}

In this paper, as in [17] and $[19,20]$, we continue to study the global existence and stability of a smooth compressible viscous flow in a 3-D slowly expanded ball. The slowly expanded ball at time $t$ is described by $S_{t}=\left\{x:|x|=\sqrt{x_{1}^{2}+x_{2}^{2}+x_{3}^{2}} \leq R(t)\right\}$, where $R(t) \in$ $C^{4}[0, \infty)$ satisfies $R(0)=1, R^{\prime}(0)=0, R^{\prime \prime}(0)=0$, moreover, $R(t)=(1+h t)^{\alpha}$ holds for $t \geq 1$, here $\alpha \in(0,1)$ and $h>0$ are fixed constants. As in [19], we suppose that the movement of gases in $\Omega=\left\{(t, x): t \geq 0,|x|=\sqrt{x_{1}^{2}+x_{2}^{2}+x_{3}^{2}} \leq R(t)\right\}$ is described by 3-D compressible barotropic Navier-Stokes equations:

$$
\begin{aligned}
& \rho_{t}+\operatorname{div}(\rho u)=0, \\
& \rho u_{t}+\rho u \cdot \nabla u+\nabla P(\rho)=\mu \Delta u+(\mu+\lambda) \nabla \operatorname{div} u,
\end{aligned}
$$

\footnotetext{
*Corresponding author. Email addresses: huicheng@nju.edu.cn, 05407@njnu.edu.cn (H. C. Yin),
} lynzhung@gmail.com (L. Zhang) 


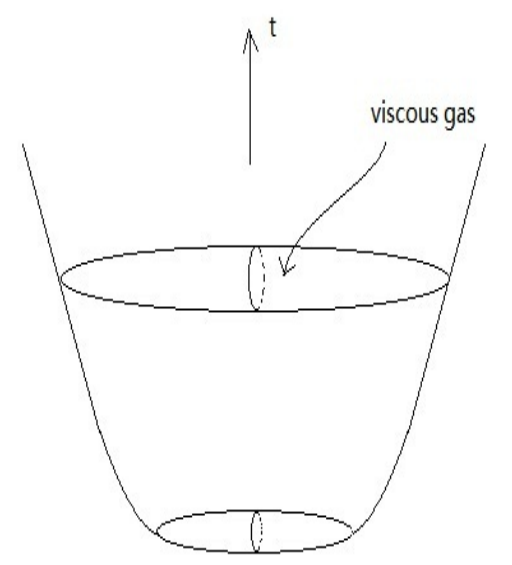

Figure 1: A viscous flow in a 3-D slowly expanded ball.

where $\rho \geq 0$ is the density, $u=\left(u_{1}, u_{2}, u_{3}\right)$ is the velocity, $\mu>0$ and $\lambda$ are the first and second viscosity coefficient respectively, $\mu+\frac{2}{3} \lambda>0$ holds, and the state equation is $P(\rho)=\rho^{\gamma}$ with $\gamma>1$.

By the physical property for the viscous flow, as in [19], one can naturally pose the following initial-boundary conditions for Eqs. (1.1a)-(1.1b)

$$
\begin{cases}\rho(0, x)=\rho_{0}(x), \quad u(0, x)=u_{0}(x), & \text { for } x \in S_{0}, \\ u(t, x)=\frac{R^{\prime}(t) x}{R(t)}, & \text { for }(t, x) \in \partial \Omega,\end{cases}
$$

where $\rho_{0}(x) \in H^{3}\left(S_{0}\right), u_{0}(x) \in H_{0}^{3}\left(S_{0}\right), \rho_{0}(x)>0$ for $x \in S_{0}$, and $\partial \Omega=\{(t, x): t \geq 0,|x|=R(t)\}$. For Eqs. (1.1a)-(1.1b) together with (1.2), completely similar to the proof of Theorem 2.1 in [19], we can obtain a local existence result as follows:

Theorem 1.1. If $\rho_{0}(x) \in H^{3}\left(S_{0}\right), \nabla \rho_{0}(x) \in H_{0}^{1}\left(S_{0}\right), u_{0}(x) \in H_{0}^{3}\left(S_{0}\right)$, and $R(t)=(1+h t)^{\alpha}$ for $t \geq 1$, then there exist a constant $h_{0}>0$ and a small constant $\varepsilon_{0}>0$ depending only on $h_{0}$ and $\alpha$ such that when

$$
\sup _{0 \leq t \leq 1,1 \leq k \leq 4}\left|R^{(k)}(t)\right|+\left\|\rho_{0}(x)-1\right\|_{H^{3}\left(S_{0}\right)}+\left\|u_{0}(x)\right\|_{H^{3}\left(S_{0}\right)}<\varepsilon_{0} \quad \text { and } \quad 0<h<h_{0},
$$

there exists some constant $T_{*}>1$ such that Eqs. (1.1a)-(1.1b) with (1.2) have a unique local solution $(\rho, u)$ which satisfies

$$
\begin{cases}\rho \in C\left(\left[0, T_{*}\right],\right. & \left.H^{3}\left(S_{t}\right)\right) \cap C^{1}\left(\left[0, T_{*}\right], H^{2}\left(S_{t}\right)\right), \\ u \in C\left(\left[0, T_{*}\right],\right. & \left.H_{0}^{1}\left(S_{t}\right) \cap H^{3}\left(S_{t}\right)\right) \cap C^{1}\left(\left[0, T_{*}\right], H_{0}^{1}\left(S_{t}\right)\right) \cap L^{2}\left(\left[0, T_{*}\right], H^{4}\left(S_{t}\right)\right) .\end{cases}
$$

Moreover, $\rho(t, x) \geq C>0$ holds for $(t, x) \in\left[0, T^{*}\right] \times S_{t}$, and

$$
\|\rho-1\|_{C\left(\left[0, T_{*}\right], H^{3}\left(S_{t}\right)\right)}+\left\|\rho_{t}\right\|_{C\left(\left[0, T_{*}\right], H^{3}\left(S_{t}\right)\right)}+\|u\|_{C\left(\left[0, T_{*}\right], H_{0}^{1}\left(S_{t}\right) \cap H^{3}\left(S_{t}\right)\right)}+\left\|u_{t}\right\|_{C\left(\left[0, T_{*}\right], H_{0}^{1}\left(S_{t}\right)\right)} \leq C \varepsilon .
$$


Remark 1.1. It follows from the assumptions $R^{\prime \prime}(0)=0, \nabla \rho_{0}(x) \in H_{0}^{1}\left(S_{0}\right)$ and $u_{0}(x) \in$ $H_{0}^{3}\left(S_{0}\right)$ in Theorem 1.1 that the compatibility of the initial velocity $u_{0}(x)$ on the boundary $\partial S_{0}=\{x:|x|=1\}$ holds and meanwhile $u_{t}(0, x) \in H_{0}^{1}\left(S_{0}\right)$ is derived from Eq. (1.1b). This fact will play a basic role in proving the local existence result in Theorem 1.1 (one can see the details in [19]).

Based on Theorem 1.1 and the continuity argument, we will establish the following global existence result:

Theorem 1.2. Under the assumptions of Theorem 1.1, when the adiabatic exponent $\gamma$ satisfies $1<\gamma<\frac{2}{3}+\frac{1}{\alpha}$, then Eqs. (1.1a)-(1.1b) with (1.2) admit a global solution $(\rho, u)$ in $\Omega$ which fulfills

$$
\begin{aligned}
& \rho(t, x) \in C\left([0, \infty), H^{3}\left(S_{t}\right)\right) \cap C^{1}\left([0, \infty), H^{2}\left(S_{t}\right)\right), \\
& u(t, x) \in C\left([0, \infty), H^{3}\left(S_{t}\right)\right) \cap C^{1}\left([0, \infty), H^{1}\left(S_{t}\right)\right), \\
& \frac{1}{2 R^{3}(t)} \leq \rho(t, x) \leq \frac{3}{2 R^{3}(t)} \quad \text { for } t \geq 1 .
\end{aligned}
$$

Remark 1.2. The assumption of $\gamma<\frac{2}{3}+\frac{1}{\alpha}$ in Theorem 1.2 is applied to guarantee the uniform integrability of the integral $\int_{\tilde{\tau}_{0}}^{t} \tilde{\tau}^{\frac{3 \alpha(\gamma-1)-4}{1+\alpha}} d \tilde{\tau}$ in (3.67) of Section 3, which is required to derive the global a priori energy estimate for the solution of (1.1a)-(1.1b) with (1.2). In addition, we specially point out that, for the air $(\gamma \approx 1.4)$ and the polytropic gases $\left(\gamma \approx \frac{5}{3}\right)$, the assumption of $\gamma<\frac{2}{3}+\frac{1}{\alpha}$ with $0<\alpha<1$ in Theorem 1.2 is obviously fulfilled.

Remark 1.3. By Theorem 1.1 and Theorem 1.2, one can conclude that the solution of (1.1a)-(1.1b) with (1.2) does not contain vacuum state in any finite time.

So far there have been extensive studies on the global existence and behaviors of solutions to the compressible Navier-Stokes equations. For one-dimensional case, see $[8,15$, 18 ] and the references therein. For multi-dimensional case with constant viscosity coefficients, the local existence of classical solution has been established in [14] in the absence of vacuum, and the local existence of strong solutions is also shown in [1,2], when the initial density may vanish in some open sets. The global existence of classical solution was first obtained in $[12,13]$ for the initial data close to a non-vacuum state and then these results were generalized in other weighted energy spaces (one can see $[3,16]$ and so on). In addition, the author in [6] studied the global existence with discontinuous initial data. Recently, for the case when the initial density may vanish in some region, under the smallness assumption on the total energy, the authors in [7] established the global existence and uniqueness of classical solutions. For large initial data with the finite total energy and different assumptions on the adiabatic constant $\gamma$, the global existence of weak solutions was firstly established by P.L. Lions in [11] and subsequently was improved in $[4,9]$. For the Eqs. (1.1a)-(1.1b) (or including the energy equation) with suitable initial-boundary values have also been extensively studied, for examples, see $[10,13]$ and the references therein. On the other hand, the authors in [5] obtained the global existence 
of weak solution to the compressible barotropic Navier-Stokes system in a time dependent domain with slip boundary condition. Finally, we point out that the authors in [17] and $[19,20]$ have established the similar conclusions as in Theorem 1.1-Theorem 1.2 for $R(t)=1+h t$ by taking the delicate weighted energy analysis through the related special solutions. However, in the case of $0<\alpha<1$ for $R(t)=(1+h t)^{\alpha}$, it seems that the special solution of (1.1a)-(1.1b) with (1.2) is difficult to be found, moreover, the resulting linearized system is slightly different from that for $\alpha=1$. This leads to somewhat different proof of Theorem 1.2 from the main result in [19]. Here we emphasize that although the proof of Theorem 1.2 is strongly motivated by [19], we still give out all the details for reader's convenience.

The paper is arranged as follows. In Section 2, we reformulate problem (1.1a)-(1.1b) with (1.2) and cite an analogous local existence result in [19]. Moreover, some uniform weighted inequalities are derived by involved analysis. Based on the analysis in Section 2 , the uniform energy estimate will be given in Section 3 and then the proof of Theorem 1.2 is completed in Section 4.

The following notations will be used throughout this paper:

$$
\begin{aligned}
& \|f\|_{2}=\left(\int_{S_{0}}|f|^{2} d y\right)^{\frac{1}{2}}, \\
& \int_{0}^{t}\|V(\tau)\| d \tau=\int_{0}^{t}\|V(\tau, \cdot)\| d \tau, \text { and } D g \text { represents } \partial_{k} g
\end{aligned}
$$

for any $k=1,2,3$.

\section{Reformulation of (1.1a)-(1.1b) with (1.2) and some uniform weighted energy inequalities}

At first, as in [19], we take a transformation of the variables $(t, x)$ as follows

$$
\left\{\begin{array}{l}
\tau=t, \\
y=\frac{x}{R(t)} .
\end{array}\right.
$$

In this case, $\Omega$ is changed into the domain $[0, \infty) \times S_{0}$, and (1.1a)-(1.1b) have such new forms in the coordinates $(\tau, y) \in[0, \infty) \times S_{0}$

$$
\left\{\begin{array}{l}
\rho_{\tau}-\frac{R^{\prime}}{R} y \cdot \nabla \rho+\frac{1}{R} \operatorname{div}(\rho u)=0, \\
(\rho u)_{\tau}-\frac{R^{\prime}}{R} y \cdot \nabla(\rho u)+\frac{1}{R} \operatorname{div}(\rho u \otimes u)+\frac{1}{R} \nabla P(\rho)=\frac{1}{R^{2}}(\mu \Delta u+(\mu+\lambda) \nabla \operatorname{divu}),
\end{array}\right.
$$


where and below all the derivatives $\nabla$ and div are about the variable $y$. Let $\phi=R^{3} \rho$ and $v=u-R^{\prime}(t) y$. Then (2.2) is changed as

$$
\begin{aligned}
& \phi_{\tau}+\frac{1}{R} \operatorname{div}(\phi v)=0, \\
& \phi v_{\tau}+\phi R^{\prime \prime} y+\phi v \cdot \frac{1}{R} \nabla v+\frac{1}{R} \phi R^{\prime} v+R^{2} \nabla P(\rho)=R(\mu \Delta u+(\mu+\lambda) \nabla \operatorname{div} u) .
\end{aligned}
$$

Meanwhile, the initial-boundary condition (1.2) becomes

$$
\begin{cases}\phi(0, y)=\rho_{0}(y), \quad v(0, y)=u_{0}(y), & y \in S_{0} \\ v(\tau, y)=0 & \text { on }[0, \infty) \times \partial S_{0} .\end{cases}
$$

By completely analogous proof of Theorem 2.1 in [19], one can obtain Theorem 1.1 (here we omit the details). Next, we start to establish some global a priori energy estimates on the solutions of (2.3a)-(2.3b) with (2.4). Let $s=\frac{3}{2}(\gamma-1)$ and $w=\phi-1$. Then (2.3a)-(2.3b) can be written as

$$
\begin{aligned}
& w_{\tau}+\frac{1}{R} d i v v=\tilde{f}, \\
& v_{\tau}+\frac{R^{\prime}}{R} v+\frac{\gamma}{R^{2 s+1}} \nabla w-R L v=\tilde{g},
\end{aligned}
$$

where

$$
\begin{aligned}
& \tilde{f}=-\frac{1}{R} \operatorname{div}(w v), \\
& L v=\mu \Delta v+(\mu+\lambda) \nabla d i v v, \\
& \tilde{g}=-\frac{1}{R} v \cdot \nabla v-R^{\prime \prime}(t) y-\frac{1}{R^{2 s+1}} \nabla P_{1}(w)-\frac{w}{1+w} R L v,
\end{aligned}
$$

and

$$
P_{1}(w)=w^{2} \int_{0}^{1} \gamma(\gamma-2)(1+\theta w)^{\gamma-3} d \theta .
$$

On the other hand, setting

$$
\tilde{\tau}=(1+h t)^{1+\alpha}, \quad m=\frac{2 \alpha}{1+\alpha} \quad \text { and } \quad s^{\prime}=\frac{3 \alpha(\gamma-1)}{2(1+\alpha)},
$$

we then have that for $\tilde{\tau} \geq \tilde{\tau}_{0} \equiv(1+h)^{1+\alpha}$,

$$
\begin{aligned}
& (1+\alpha) h \partial_{\tilde{\tau}} w+\tilde{\tau}^{-m} d i v v=f, \\
& (1+\alpha) h \partial_{\tilde{\tau}} v+\frac{\alpha h}{\tilde{\tau}} v-L v+\frac{\gamma}{\tilde{\tau}^{2 s^{\prime}+m}} \nabla w=g, \\
& \left.w(\tilde{\tau}, y)\right|_{\tilde{\tau}=\tilde{\tau}_{0}}=w_{0}(y) \equiv \phi\left(\tilde{\tau}_{0}, y\right)-1,\left.\quad v(\tilde{\tau}, y)\right|_{\tilde{\tau}=\tilde{\tau}_{0}}=v_{0}(y) \equiv v\left(\tilde{\tau}_{0}, y\right), \\
& v=0 \quad \text { on }\left[\tilde{\tau}_{0},+\infty\right) \times \partial S_{0},
\end{aligned}
$$


where

$$
\begin{aligned}
& f=-\frac{1}{\tilde{\tau}^{m}} \operatorname{div}(v w) \\
& L v=\mu \Delta v+(\mu+\lambda) \nabla d i v v, \\
& g=-\frac{1}{\tilde{\tau}^{m}} v \cdot \nabla v-\frac{1}{\tilde{\tau}^{m+2 s^{\prime}}} \nabla P_{1}(w)+\alpha(1-\alpha) h^{2} \tilde{\tau}^{-\frac{2}{1+\alpha}} y-\frac{w}{1+w} L v .
\end{aligned}
$$

We now derive a series of basic energy estimates on $(w, v)$. Set

$$
f_{0}=-\frac{w}{\tilde{\tau}^{m}} \operatorname{div} v \quad \text { and } \quad \frac{d \varphi}{d \tilde{\tau}}=(1+\alpha) h \varphi_{\tilde{\tau}}+\frac{1}{\tilde{\tau}^{m}} v \cdot \nabla \varphi .
$$

Then we have

Lemma 2.1 (Weight $L^{2}$-estimate of $(w, v)$ ). For small $h>0$ and $t \geq \tilde{\tau}_{0}$, one has

$$
\begin{aligned}
& h\left(\|w\|_{2}^{2}+\left\|\tilde{\tau}^{s^{\prime}} v\right\|_{2}^{2}\right)+\int_{\tilde{\tau}_{0}}^{t}\left(\left\|\tilde{\tau}^{s^{\prime}} \nabla v\right\|_{2}^{2}+\left\|\tilde{\tau}^{s^{\prime}+m} \frac{d w}{d \tau}\right\|_{2}^{2}\right) d \tilde{\tau} \\
\leq & C\left(h\left\|\left(w_{0}, v_{0}\right)\right\|_{2}^{2}+\int_{\tilde{\tau}_{0}}^{t}\left(|(f, w)|+\left|\left(\tilde{\tau}^{2 s^{\prime}} g, v\right)\right|+\left\|\tilde{\tau}^{s^{\prime}+m} f_{0}\right\|_{2}^{2}\right) d \tilde{\tau}\right) .
\end{aligned}
$$

Proof. By $\int_{S_{0}}(2.5 \mathrm{a}) \times \gamma w d y$ and $\int_{S_{0}} \tilde{\tau}^{2 s^{\prime}}(2.5 \mathrm{~b}) \cdot v d y$, we have

$$
\frac{1}{2}(1+\alpha) \gamma h \partial_{\tilde{\tau}}\|w\|_{2}^{2}+\frac{\gamma}{\tilde{\tau}^{m}}(\operatorname{div} v, w)=(\gamma f, w)
$$

and

$$
\left((1+\alpha) h \tilde{\tau}^{2 s^{\prime}} \partial_{\tilde{\tau}} v, v\right)+\alpha h \tilde{\tau}^{2 s^{\prime}-1}(v, v)-\tilde{\tau}^{2 s^{\prime}}(L v, v)+\frac{\gamma}{\tilde{\tau}^{m}}(\nabla w, v)=\left(\tilde{\tau}^{2 s^{\prime}} v, g\right) .
$$

Note that

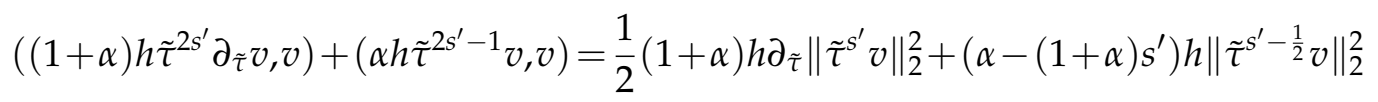

and

$$
-\left(\tilde{\tau}^{2 s^{\prime}} L v, v\right)=\mu\left\|\tilde{\tau}^{s^{\prime}} \nabla v\right\|_{2}^{2}+(\mu+\lambda)\left\|\tilde{\tau}^{s^{\prime}} \operatorname{div}\right\|_{2}^{2} .
$$

Then substituting the above two equalities into (2.8) and subsequently adding (2.8) to (2.7), we have

$$
\begin{aligned}
& \frac{1}{2}(1+\alpha) \frac{d}{d \tilde{\tau}}\left(h \gamma\|w\|_{2}^{2}+h\left\|\tilde{\tau}^{s^{\prime}} v\right\|_{2}^{2}\right)+\mu\left\|\tilde{\tau}^{s^{\prime}} \nabla v\right\|_{2}^{2}+(\mu+\lambda)\left\|\tilde{\tau}^{s^{\prime}} d i v v\right\|_{2}^{2} \\
= & \left(\alpha-(1+\alpha) s^{\prime}\right) h\left\|\tilde{\tau}^{s^{\prime}-\frac{1}{2}} v\right\|_{2}^{2}+(\gamma f, w)+\left(\tilde{\tau}^{2 s^{\prime}} g, v\right) .
\end{aligned}
$$


By Poincare inequality for $v$, we see that

$$
\left\|\tilde{\tau}^{s^{\prime}-\frac{1}{2}} v\right\|_{2}^{2} \leq C\left\|\tilde{\tau}^{s^{\prime}} \nabla v\right\|_{2}^{2}
$$

Combining (2.10) with (2.9) yields that for small $h>0$,

$$
\frac{d}{d \tilde{\tau}}\left(h \gamma\|w\|_{2}^{2}+h\left\|\tilde{\tau}^{s^{\prime}} v\right\|_{2}^{2}\right)+C\left\|\tilde{\tau}^{s^{\prime}} \nabla v\right\|_{2}^{2} \leq(\gamma f, w)+\left(\tilde{\tau}^{2 s^{\prime}} g, v\right) .
$$

Integrating (2.11) with respect to the variable $\tilde{\tau}$ over $\left(\tilde{\tau}_{0}, t\right)$ derives

$$
\begin{aligned}
& h\left(\|w\|_{2}^{2}+\left\|\tilde{\tau}^{s^{\prime}} v\right\|_{2}^{2}\right)+\int_{\tilde{\tau}_{0}}^{t}\left\|\tilde{\tau}^{s^{\prime}} \nabla v\right\|_{2}^{2} d \tilde{\tau} \\
\leq & C h\left\|\left(w_{0}, v_{0}\right)\right\|_{2}^{2}+C \int_{\tilde{\tau}_{0}}^{t}\left(|(\gamma f, w)|+\left|\left(\tilde{\tau}^{2 s^{\prime}} g, v\right)\right|\right) d \tilde{\tau} .
\end{aligned}
$$

In addition, one has that from (2.5a)

$$
\frac{d w}{d \tilde{\tau}}=-\frac{1}{\tilde{\tau}^{m}} \operatorname{div} v+f_{0}
$$

which easily implies

$$
\left\|\tilde{\tau}^{s^{\prime}+m} \frac{d w}{d \tilde{\tau}}\right\|_{2}^{2} \leq 2\left\|\tilde{\tau}^{s^{\prime}} d i v v\right\|_{2}^{2}+2\left\|\tilde{\tau}^{s^{\prime}+m} f_{0}\right\|_{2}^{2}
$$

From (2.12)-(2.13), we can obtain (2.6).

Lemma 2.2 (Weighted $L^{2}$-estimate of $\nabla v$ ). For $t \geq \tilde{\tau}_{0}$, we have

$$
\begin{aligned}
& h\left\|\tilde{\tau}^{s^{\prime}} \nabla v\right\|_{2}^{2}+\int_{\tilde{\tau}_{0}}^{t}\left(\left\|h w_{\tilde{\tau}}\right\|_{2}^{2}+\left\|h \tilde{\tau}^{s^{\prime}} v_{\tilde{\tau}}\right\|_{2}^{2}\right) d \tilde{\tau} \\
\leq & \eta_{1} h^{2} \int_{\tilde{\tau}_{0}}^{t}\left\|\frac{\nabla w}{\tilde{\tau}^{s^{\prime}+1+m}}\right\|_{2}^{2} d \tilde{\tau}+\frac{C h}{\eta_{1}}\left\|\left(w_{0}, v_{0}\right)\right\|_{H^{1}}^{2}+\frac{C}{\eta_{1}} \int_{\tilde{\tau}_{0}}^{t} A_{1} d \tilde{\tau}+\int_{\tilde{\tau}_{0}}^{t} A_{2} d \tilde{\tau},
\end{aligned}
$$

where $0<\eta_{1}<1$ is a small fixed constant, $A_{1}=|(\gamma f, w)|+\left|\left(\tilde{\tau}^{2 s^{\prime}} g, v\right)\right|$, and $A_{2}=\left|\left(\gamma f, h w_{\tilde{\tau}}\right)\right|+$ $\left|\left(\tilde{\tau}^{2 s^{\prime}} g, h v_{\tilde{\tau}}\right)\right|$.

Proof. Computing $\int_{S_{0}}(2.5 \mathrm{a}) \times \gamma h w_{\tilde{\tau}} d y$ and $\int_{S_{0}} \tilde{\tau}^{s^{\prime}}(2.5 \mathrm{~b}) \cdot h v_{\tilde{\tau}} d y$ yields

$$
(1+\alpha) \gamma\left\|h \partial_{\tilde{\tau}} w\right\|_{2}^{2}+\left(\frac{\gamma}{\tilde{\tau}^{m}} d i v v, h w_{\tilde{\tau}}\right)=\left(\gamma f, h w_{\tilde{\tau}}\right)
$$

and

$$
(1+\alpha)\left\|h \tilde{\tau}^{s^{\prime}} \partial_{\tilde{\tau}} v\right\|_{2}^{2}+\alpha h\left(\tilde{\tau}^{2 s^{\prime}-1} v, h v_{\tilde{\tau}}\right)-\left(\tilde{\tau}^{2 s^{\prime}} L v, h v_{\tilde{\tau}}\right)+\left(\frac{\gamma}{\tilde{\tau}^{m}} \nabla w, h v_{\tilde{\tau}}\right)=\left(\tilde{\tau}^{2 s^{\prime}} g, h v_{\tilde{\tau}}\right) .
$$


Next we treat the terms in the left hand sides of (2.15)-(2.16) separately. For the term $-\left(\tilde{\tau}^{2 s^{\prime}} L v, h v_{\tilde{\tau}}\right)$, we have

$$
\begin{aligned}
& -\left(\tilde{\tau}^{2 s^{\prime}} L v, h v_{\tilde{\tau}}\right) \\
= & h \frac{\mu}{2} \partial_{\tilde{\tau}}\left\|\tilde{\tau}^{s^{\prime}} \nabla v\right\|_{2}^{2}+h \frac{\mu+\lambda}{2} \partial_{\tilde{\tau}}\left\|\tilde{\tau}^{s^{\prime}} d i v v\right\|_{2}^{2} \\
& -h s^{\prime} \mu\left\|\tilde{\tau}^{s^{\prime}-\frac{1}{2}} \nabla v\right\|_{2}^{2}-h s^{\prime}(\mu+\lambda)\left\|\tilde{\tau}^{s^{\prime}-\frac{1}{2}} d i v v\right\|_{2}^{2} .
\end{aligned}
$$

For the terms $\alpha h\left(\tilde{\tau}^{2 s^{\prime}-1} v, h v_{\tilde{\tau}}\right)$ and $\left(\frac{\gamma}{\tilde{\tau}^{m}} d i v v, h w_{\tilde{\tau}}\right)$, one has

$$
\begin{aligned}
& \alpha h\left(\tilde{\tau}^{2 s^{\prime}-1} v, h v_{\tilde{\tau}}\right) \leq \frac{1}{2}\left\|h \tilde{\tau}^{s^{\prime}} v\right\|_{2}^{2}+\frac{1}{2}\left\|\tilde{\tau}^{s^{\prime}-1} h v_{\tilde{\tau}}\right\|_{2}^{2}, \\
& \left(\frac{\gamma}{\tilde{\tau}^{m}} d i v v, h w_{\tilde{\tau}}\right) \leq \frac{\gamma}{4}\left\|h w_{\tilde{\tau}}\right\|_{2}^{2}+\gamma\left\|\frac{1}{\tilde{\tau}^{m}} d i v v\right\|_{2}^{2} .
\end{aligned}
$$

For the term $\left(\frac{\gamma}{\tilde{\tau}^{m}} \nabla w, h v_{\tilde{\tau}}\right)$, we have

$$
\left(\frac{\gamma}{\tilde{\tau}^{m}} \nabla w, h v_{\tilde{\tau}}\right)=-\partial_{\tilde{\tau}}\left(h \frac{\gamma}{\tilde{\tau}^{m}} w, d i v v\right)+m \frac{\gamma}{\tilde{\tau}^{1+m}}(\nabla w, h v)+\left(\frac{\gamma}{\tilde{\tau}^{m}} h \partial_{\tilde{\tau}} w, d i v v\right) .
$$

This, together with Holder's inequality, yields for small $\eta_{1}>0$,

$$
\begin{aligned}
& \left(\frac{\gamma}{\tilde{\tau}} \nabla w, v_{\tilde{\tau}}\right) \\
\geq & -\frac{d}{d \tilde{\tau}}\left(\frac{h \gamma}{\tilde{\tau}^{m}} w, d i v v\right)-\eta_{1} h^{2}\left\|\frac{\nabla w}{\tilde{\tau}^{s^{\prime}}+1+m}\right\|_{2}^{2}-\frac{C}{\eta_{1}}\left\|\tilde{\tau}^{s^{\prime}} v\right\|_{2}^{2}-\frac{\gamma}{4}\left\|h w_{\tilde{\tau}}\right\|_{2}^{2}-\gamma\left\|\frac{1}{\tilde{\tau}^{m}} d i v v\right\|_{2}^{2} .
\end{aligned}
$$

Substituting (2.17)-(2.19) into (2.15)-(2.16), we arrive at

$$
\begin{aligned}
& \gamma\left\|h \partial_{\tilde{\tau}} w\right\|_{2}^{2}+\left\|h \tilde{\tau}^{s^{\prime}} \partial_{\tilde{\tau}} v\right\|_{2}^{2}+h \partial_{\tilde{\tau}}\left(\frac{\mu}{2}\left\|\tilde{\tau}^{s^{\prime}} \nabla v\right\|_{2}^{2}+\frac{\mu+\lambda}{2}\left\|\tilde{\tau}^{s^{\prime}} d i v v\right\|_{2}^{2}-\left(\frac{\gamma}{\tilde{\tau}^{m}} w, d i v v\right)\right) \\
\leq & \eta_{1} h^{2}\left\|\frac{\nabla w}{\tilde{\tau}^{s^{\prime}+1+m}}\right\|_{2}^{2}+\frac{C}{\eta_{1}}\left\|\tilde{\tau}^{s^{\prime}} \nabla v\right\|_{2}^{2}+C\left(\left(\gamma f, h w_{\tilde{\tau}}\right)+\left(\tilde{\tau}^{2 s^{\prime}} g, h v_{\tilde{\tau}}\right)\right) .
\end{aligned}
$$

Note that

$$
-\left(\frac{\gamma}{\tilde{\tau}^{m}} w, \operatorname{div} v\right) \leq \frac{\mu}{4}\left\|\frac{1}{\tilde{\tau}^{m}} \operatorname{div} v\right\|_{2}^{2}+\frac{\gamma^{2}}{\mu}\|w\|_{2}^{2} .
$$

Together with $\int_{\tilde{\tau}_{0}}^{t}\left(\frac{C}{\eta_{1}} \times(2.11)+(2.20)\right) d \tilde{\tau}$, this yields

$$
\begin{aligned}
& \quad h\left\|\tilde{\tau}^{s^{\prime}} \nabla v\right\|_{2}^{2}+\int_{\tilde{\tau}_{0}}^{t}\left(\left\|h w_{\tilde{\tau}}\right\|_{2}^{2}+\left\|h \tilde{\tau}^{s^{\prime}} v_{\tilde{\tau}}\right\|_{2}^{2}\right) d \tilde{\tau} \\
& \leq \int_{\tilde{\tau}_{0}}^{t} \eta_{1} h^{2}\left\|\frac{\nabla w}{\tilde{\tau}^{s^{\prime}+1+m}}\right\|_{2}^{2} d \tilde{\tau}+\frac{C}{\eta_{1}}\left\|\left(w_{0}, v_{0}\right)\right\|_{H^{1}}^{2} \\
& \quad+\int_{\tilde{\tau}_{0}}^{t}\left(\left(\gamma f, h w_{\tilde{\tau}}\right)+\left(\tilde{\tau}^{2 s^{\prime}} g, h v_{\tilde{\tau}}\right)\right) d \tilde{\tau}+\frac{C}{\eta_{1}} \int_{\tilde{\tau}_{0}}^{t}\left((\gamma f, w)+\left(\tilde{\tau}^{2 s^{\prime}} g, v\right)\right) d \tilde{\tau},
\end{aligned}
$$

which completes the proof of Lemma 2.2. 
Lemma 2.3 (Weighted $L^{2}$-estimate of $\left.\left(w_{\tilde{\tau}}, v_{\tilde{\tau}}\right)\right)$. For $t \geq \tilde{\tau}_{0}$, we have

$$
\begin{aligned}
& h\left\|h w_{\tilde{\tau}}\right\|_{2}^{2}+h\left\|h \tilde{\tau}^{s^{\prime}} v_{\tilde{\tau}}\right\|_{2}^{2}+\int_{\tilde{\tau}_{0}}^{t}\left\|h \tilde{\tau}^{s^{\prime}} \nabla v_{\tilde{\tau}}\right\|_{2}^{2} d \tilde{\tau} \\
\leq & C\left(\eta_{2}\right) h\left\|\left(w_{0}, v_{0}\right)\right\|_{H^{2}}^{2}+c \eta_{2} h^{2} \int_{\tilde{\tau}_{0}}^{t}\left\|\frac{\nabla w}{\tilde{\tau}^{s^{\prime}}+1+m}\right\|_{2}^{2} d \tilde{\tau}+C\left(\eta_{2}\right) \int_{\tilde{\tau}_{0}}^{t}\left(A_{1}+A_{2}+A_{3}\right) d \tilde{\tau},
\end{aligned}
$$

where $A_{3}=\left|\left(\gamma h f_{\tilde{\tau}}, h w_{\tilde{\tau}}\right)\right|+\left|\left(\tilde{\tau}^{2 s^{\prime}} h g_{\tilde{\tau}}, h v_{\tilde{\tau}}\right)\right|$.

Proof. Computing $\int_{S_{0}} \partial_{\tilde{\tau}}(2.5 \mathrm{a}) \times \gamma h^{2} w_{\tilde{\tau}} d y$, we have

$$
\frac{1}{2}(1+\alpha) h \gamma \frac{d}{d \tilde{\tau}}\left\|h w_{\tilde{\tau}}\right\|_{2}^{2}+\left(\frac{\gamma}{\tilde{\tau}^{m}} h d i v v_{\tilde{\tau}}, h w_{\tilde{\tau}}\right)-m\left(\frac{\gamma}{\tilde{\tau}^{1+m}} h d i v v, h w_{\tilde{\tau}}\right)=\left(\gamma h f_{\tilde{\tau}}, h w_{\tilde{\tau}}\right) .
$$

Computing $\int_{S_{0}} \tilde{\tau}^{2 s^{\prime}} h \partial_{\tilde{\tau}}(2.5 \mathrm{~b}) \cdot h v_{\tilde{\tau}} d y$ yields

$$
\begin{aligned}
& \frac{1}{2}(1+\alpha) h \partial_{\tilde{\tau}}\left\|h \tilde{\tau}^{s^{\prime}} v_{\tilde{\tau}}\right\|_{2}^{2}+\left(\alpha-s^{\prime}(1+\alpha)\right) h\left\|h \tilde{\tau}^{s^{\prime}-\frac{1}{2}} v_{\tilde{\tau}}\right\|_{2}^{2}-\alpha\left(h \tilde{\tau}^{2 s^{\prime}-2} v, h v_{\tilde{\tau}}\right)-\left(h L v_{\tilde{\tau}}, \tilde{\tau}^{2 s^{\prime}} h v_{\tilde{\tau}}\right) \\
& +\left(\frac{\gamma}{\tilde{\tau}^{m}} h \nabla w_{\tilde{\tau}}, h v_{\tilde{\tau}}\right)-\left(2 s^{\prime}+m\right)\left(\frac{\gamma}{\tilde{\tau}^{1+m}} h \nabla w, h v_{\tilde{\tau}}\right)=\left(\tilde{\tau}^{2 s^{\prime}} h g_{\tilde{\tau}}, h v_{\tilde{\tau}}\right) .
\end{aligned}
$$

Noting that for small $\eta_{2}>0$,

$$
\begin{aligned}
& -\left(h L v_{\tilde{\tau}}, \tilde{\tau}^{2 s^{\prime}} h v_{\tilde{\tau}}\right)=\mu\left\|h \tilde{\tau}^{s^{\prime}} \nabla v_{\tilde{\tau}}\right\|_{2}^{2}+(\mu+\lambda)\left\|h \tilde{\tau}^{s^{\prime}} d i v v_{\tilde{\tau}}\right\|_{2}^{2} \\
& \left(2 s^{\prime}+m\right)\left(\frac{\gamma}{\tilde{\tau}^{1+m}} h \nabla w, h v_{\tilde{\tau}}\right) \leq \eta_{2} h^{2}\left\|\frac{\nabla w}{\tilde{\tau}^{s^{\prime}+1+m}}\right\|_{2}^{2}+\frac{C}{\eta_{2}}\left\|h \tilde{\tau}^{s^{\prime}} v_{\tilde{\tau}}\right\|_{2}^{2},
\end{aligned}
$$

and

$$
\left(\frac{\gamma}{\tilde{\tau}^{1+m}} h d i v v, h w_{\tilde{\tau}}\right) \leq\left\|h w_{\tilde{\tau}}\right\|_{2}^{2}+\frac{\gamma^{2}}{4}\left\|h \tilde{\tau}^{s^{\prime}} \operatorname{div}\right\|_{2}^{2} .
$$

Then substituting this and (2.24a)-(2.24b) into (2.23), and subsequently combining with (2.22), we arrive at

$$
\begin{aligned}
& \quad \frac{d}{d \tilde{\tau}}\left(h\left\|h w_{\tilde{\tau}}\right\|_{2}^{2}+h\left\|h \tilde{\tau}^{s^{\prime}} v_{\tilde{\tau}}\right\|_{2}^{2}\right)+\left\|\tilde{\tau}^{s^{\prime}} h \nabla v_{\tilde{\tau}}\right\|_{2}^{2} \\
& \leq \eta_{2} h^{2}\left\|\frac{\nabla w}{\tilde{\tau}^{s^{\prime}}+1+m}\right\|_{2}^{2}+\frac{C}{\eta_{2}}\left(\left\|\tilde{\tau}^{s^{\prime}} h v_{\tilde{\tau}}\right\|_{2}^{2}+\left\|h w_{\tilde{\tau}}\right\|_{2}^{2}\right) \\
& \quad+C\left\|\tilde{\tau}^{s^{\prime}} h \nabla v\right\|_{2}^{2}+C\left(\left|\left(\gamma h f_{\tilde{\tau}}, h w_{\tilde{\tau}}\right)\right|+\left|\left(h \tilde{\tau}^{2 s^{\prime}} g_{\tilde{\tau}}, h v_{\tilde{\tau}}\right)\right|\right),
\end{aligned}
$$

here we have applied such a fact

$$
\left|h\left(h \tilde{\tau}^{2 s^{\prime}-2} v, h v_{\tilde{\tau}}\right)\right| \leq h\left\|h \tilde{\tau}^{s^{\prime}} v_{\tilde{\tau}}\right\|_{2}^{2}+C h\left\|\tilde{\tau}^{s^{\prime}} h \nabla v\right\|_{2}^{2}
$$


Integrating $(2.25)$ over $\left(\tilde{\tau}_{0}, t\right)$ with respect to the variable $\tilde{\tau}$, one has

$$
\begin{aligned}
& \quad h\left\|h w_{\tilde{\tau}}\right\|_{2}^{2}+h\left\|h \tilde{\tau}^{s^{\prime}} v_{\tilde{\tau}}\right\|_{2}^{2}+\int_{\tilde{\tau}_{0}}^{t}\left\|h \tilde{\tau}^{s^{\prime}} \nabla v_{\tilde{\tau}}\right\|_{2}^{2} d \tilde{\tau} \\
& \leq C h\left\|\left(w_{0}, v_{0}\right)\right\|_{H^{2}}^{2}+\eta_{2} h^{2} \int_{\tilde{\tau}_{0}}^{t}\left\|\frac{\nabla w}{\tilde{\tau}^{s^{\prime}+1+m}}\right\|_{2}^{2} d \tilde{\tau}+\frac{C}{\eta_{2}} \int_{\tilde{\tau}_{0}}^{t}\left(\left\|h \tilde{\tau}^{s^{\prime}} v_{\tilde{\tau}}\right\|_{2}^{2}+\left\|h w_{\tilde{\tau}}\right\|_{2}^{2}\right) d \tilde{\tau} \\
& \quad+C \int_{\tilde{\tau}_{0}}^{t}\left(\left\|\tilde{\tau}^{s^{\prime}} h \nabla v\right\|_{2}^{2}+\left(\left|\left(\gamma h f_{\tilde{\tau}}, h w_{\tilde{\tau}}\right)\right|+\left|\left(\tilde{\tau}^{2 s^{\prime}} h g_{\tilde{\tau}}, h v_{\tilde{\tau}}\right)\right|\right)\right) d \tilde{\tau} .
\end{aligned}
$$

Computing $\frac{C}{\eta_{2}} \times(2.14)+C \times(2.6)+(2.26)$, we see that

$$
\begin{aligned}
& h\left\|h w_{\tilde{\tau}}\right\|_{2}^{2}+h\left\|h \tilde{\tau}^{s^{\prime}} v_{\tilde{\tau}}\right\|_{2}^{2}+\int_{\tilde{\tau}_{0}}^{t}\left\|h \tilde{\tau}^{s^{\prime}} \nabla v_{\tilde{\tau}}\right\|_{2}^{2} d \tilde{\tau} \\
& \leq C h\left\|\left(w_{0}, v_{0}\right)\right\|_{H^{2}}^{2}+\frac{C h}{\eta_{1} \eta_{2}} \|\left(w_{0}, v_{0} \|_{H^{1}}^{2}\right. \\
& \quad+\frac{C}{\eta_{1} \eta_{2}} \int_{\tilde{\tau}_{0}}^{t} A_{1} d \tilde{\tau}+\frac{C}{\eta_{2}} \int_{\tilde{\tau}_{0}}^{t} A_{2} d \tilde{\tau}+\left(\frac{C}{\eta_{2}} \eta_{1}+\eta_{2}\right) h^{2} \int_{\tilde{\tau}_{0}}^{t}\left\|\frac{\nabla w}{\tilde{\tau}^{s^{\prime}+1+m}}\right\|_{2}^{2} d \tilde{\tau} \\
& \quad+C \int_{\tilde{\tau}_{0}}^{t}\left(\left|\left(\gamma h f_{\tilde{\tau}}, h w_{\tilde{\tau}}\right)\right|+\left|\left(\tilde{\tau}^{2 s^{\prime}} h g_{\tilde{\tau}}, h v_{\tilde{\tau}}\right)\right|\right) d \tilde{\tau} .
\end{aligned}
$$

Set $\eta_{1}=\eta_{2}^{2}$, we then complete the proof of Lemma 2.3.

Lemma 2.4 (Weighted $L^{2}$-estimate of $\nabla v_{\tilde{\tau}}$ ). For $t \geq \tilde{\tau}_{0}$ and $0<\eta_{3}<1$, we have

$$
\begin{aligned}
& \quad h\left\|\tilde{\tau}^{s^{\prime}} h \nabla v_{\tilde{\tau}}\right\|_{2}^{2}+\int_{\tilde{\tau}_{0}}^{t}\left(\left\|h^{2} w_{\tilde{\tau} \tilde{\tau}}\right\|_{2}^{2}+\left\|h^{2} \tilde{\tau}^{s^{\prime}} v_{\tilde{\tau} \tilde{\tau}}\right\|_{2}^{2}\right) d \tilde{\tau} \\
& \leq C h\left\|v_{\tilde{\tau}}\left(\tilde{\tau}_{0}\right)\right\|_{H^{1}}^{2}+C\left(\eta_{3}\right) h\left\|\left(w_{0}, v_{0}\right)\right\|_{H^{2}}^{2}+C \eta_{3} h^{2} \int_{\tilde{\tau}_{0}}^{t}\left\|\frac{\nabla w}{\tilde{\tau}^{s^{\prime}+m+1}}\right\|_{2}^{2} d \tilde{\tau} \\
& \quad+C\left(\eta_{3}\right) \int_{\tilde{\tau}_{0}}^{t}\left(A_{1}+A_{2}+A_{3}+A_{4}\right) d \tilde{\tau}
\end{aligned}
$$

where $A_{4}=\left|\left(\gamma h f_{\tilde{\tau}}, h^{2} w_{\tilde{\tau} \tilde{\tau}}\right)\right|+\left|\left(\tilde{\tau}^{s s^{\prime}} h g_{\tilde{\tau}}, h^{2} v_{\tilde{\tau} \tau}\right)\right|$.

Proof. Computing $\int_{S_{0}} \partial_{\tilde{\tau}}(2.5 \mathrm{a}) \times \gamma h^{3} w_{\tilde{\tau} \tilde{\tau}} d y$ and $\int_{S_{0}} \tilde{\tau}^{2 s^{\prime}} h \partial_{\tilde{\tau}}(2.5 \mathrm{~b}) \cdot h^{2} v_{\tilde{\tau} \tilde{\tau}} d y$ yields respectively,

$$
(1+\alpha) \gamma\left\|h^{2} w_{\tilde{\tau} \tilde{\tau}}\right\|_{2}^{2}+\left(\frac{h \gamma}{\tilde{\tau}^{m}} \operatorname{div}_{\tilde{\tau}}, h^{2} w_{\tilde{\tau} \tilde{\tau}}\right)-m\left(\frac{h \gamma}{\tilde{\tau}^{1+m}} \operatorname{div}, h^{2} w_{\tilde{\tau} \tilde{\tau}}\right)=\left(\gamma h f_{\tilde{\tau}}, h^{2} w_{\tilde{\tau} \tilde{\tau}}\right)
$$

and

$$
\begin{aligned}
& (1+\alpha)\left\|h^{2} \tilde{\tau}^{s^{\prime}} v_{\tilde{\tau} \tilde{\tau}}\right\|_{2}^{2}+h \tilde{\tau}^{2 s^{\prime}-1}\left(h v_{\tilde{\tau}}, h^{2} v_{\tilde{\tau} \tilde{\tau}}\right)-h \tilde{\tau}^{2 s^{\prime}-2}\left(h v, h^{2} v_{\tilde{\tau} \tilde{\tau}}\right)-\left(\tilde{\tau}^{2 s^{\prime}} h L v_{\tilde{\tau}}, h^{2} v_{\tilde{\tau} \tilde{\tau}}\right) \\
& +\left(\frac{h \gamma}{\tilde{\tau}^{m}} \nabla w_{\tilde{\tau}}, h^{2} v_{\tilde{\tau} \tilde{\tau}}\right)-\left(2 s^{\prime}+m\right)\left(\frac{h \gamma}{\tilde{\tau}^{1+m}} \nabla w, h^{2} v_{\tilde{\tau} \tilde{\tau}}\right)=\left(\tilde{\tau}^{2 s^{\prime}} h g_{\tilde{\tau}}, h^{2} v_{\tilde{\tau} \tilde{\tau}}\right) .
\end{aligned}
$$


We now treat the terms in (2.29) separately. For the term $-\left(\tilde{\tau}^{2 s^{\prime}} h L v_{\tilde{\tau}}, h^{2} v_{\tilde{\tau} \tau}\right)$,

$$
\begin{aligned}
& -\left(\tilde{\tau}^{2 s^{\prime}} h L v_{\tilde{\tau}}, h^{2} v_{\tilde{\tau} \tilde{\tau}}\right) \\
= & h \partial_{\tilde{\tau}}\left(\frac{\mu}{2}\left\|\tilde{\tau}^{s^{\prime}} h \nabla v_{\tilde{\tau}}\right\|_{2}^{2}+\frac{\mu+\lambda}{2}\left\|h \tilde{\tau}^{s^{\prime}} \operatorname{div}_{\tilde{\tau}}\right\|_{2}^{2}\right) \\
& \quad-s^{\prime} h \mu\left\|\tilde{\tau}^{s^{\prime}-\frac{1}{2}} h \nabla v_{\tilde{\tau}}\right\|_{2}^{2}-s^{\prime} h(\mu+\lambda)\left\|\tilde{\tau}^{s^{\prime}-\frac{1}{2}} h d i v v_{\tilde{\tau}}\right\|_{2}^{2} .
\end{aligned}
$$

For the term $\left(\frac{h \gamma}{\tilde{\tau}^{m}} \nabla w_{\tilde{\tau}}, h^{2} v_{\tilde{\tau} \tilde{\tau}}\right)$, one has

$$
\begin{aligned}
& \left(\frac{h \gamma}{\tilde{\tau}^{m}} \nabla w_{\tilde{\tau}}, h^{2} v_{\tilde{\tau} \tilde{\tau}}\right) \\
= & -\frac{d}{d \tilde{\tau}}\left(\frac{h \gamma}{\tilde{\tau}} w_{\tilde{\tau}}, h^{2} d i v v_{\tilde{\tau}}\right)-m \frac{\gamma}{\tilde{\tau}^{1+m}}\left(h w_{\tilde{\tau}}, h^{2} d i v v_{\tilde{\tau}}\right)+\frac{\gamma}{\tilde{\tau}^{m}}\left(h^{2} w_{\tilde{\tau} \tilde{\tau}}, h d i v v_{\tilde{\tau}}\right) \\
\geq & -\frac{d}{d \tilde{\tau}}\left(\frac{h \gamma}{\tilde{\tau}^{m}} w_{\tilde{\tau}}, h^{2} d i v v_{\tilde{\tau}}\right)-C h\left(\left\|h w_{\tilde{\tau}}\right\|_{2}^{2}+\left\|\frac{h}{\tilde{\tau}^{1+m}} d i v v_{\tilde{\tau}}\right\|_{2}^{2}\right)-\frac{\gamma}{2}\left\|h^{2} w_{\tilde{\tau} \tilde{\tau}}\right\|_{2}^{2} \\
& -\frac{\gamma}{2}\left\|\frac{h}{\tilde{\tau}^{m}} d i v v_{\tilde{\tau}}\right\|_{2}^{2} .
\end{aligned}
$$

For the term $-\left(\frac{h \gamma}{\tilde{\tau}^{1+m}} \nabla w, h^{2} v_{\tilde{\tau} \tilde{\tau}}\right)$, we can obtain that for $\eta_{3}>0$,

$$
\begin{aligned}
& -\left(\frac{h \gamma}{\tilde{\tau}^{1+m}} \nabla w, h^{2} v_{\tilde{\tau} \tilde{\tau}}\right) \\
= & -\frac{d}{d \tilde{\tau}}\left(\frac{h \gamma}{\tilde{\tau}^{1+m}} \nabla w, h^{2} v_{\tilde{\tau}}\right)-(1+m)\left(\frac{2 \gamma h}{\tilde{\tau}^{2+m}} \nabla w, h^{2} v_{\tilde{\tau}}\right)+\left(\frac{h \gamma}{\tilde{\tau}^{1+m}} \nabla w_{\tilde{\tau}}, h^{2} v_{\tilde{\tau}}\right) \\
\geq & -\frac{d}{d \tilde{\tau}}\left(\frac{h \gamma}{\tilde{\tau}^{1+m}} \nabla w, h^{2} v_{\tilde{\tau}}\right)-\eta_{3} h^{2}\left\|\frac{\nabla w}{\tilde{\tau}^{s^{\prime}+m+2}}\right\|_{2}^{2}-\frac{C}{\eta_{3}}\left\|\tilde{\tau}^{s^{\prime}} h^{2} \nabla v_{\tilde{\tau}}\right\|_{2}^{2}-\left\|h w_{\tilde{\tau}}\right\|_{2}^{2} .
\end{aligned}
$$

Substituting (2.30)-(2.32) into (2.29) and then combining with (2.28) yield

$$
\begin{aligned}
& \gamma\left\|h^{2} w_{\tilde{\tau} \tilde{\tau}}\right\|_{2}^{2}+\left\|h^{2} \tilde{\tau}^{s^{\prime}} v_{\tilde{\tau} \tilde{\tau}}\right\|_{2}^{2}+h \partial_{\tilde{\tau}}\left(\frac{\mu}{2}\left\|\tilde{\tau}^{s^{\prime}} h \nabla v_{\tilde{\tau}}\right\|_{2}^{2}+\frac{\mu+\lambda}{2}\left\|h \tilde{\tau}^{s^{\prime}} d i v v_{\tilde{\tau}}\right\|_{2}^{2}\right. \\
& \left.-\left(\frac{h \gamma}{\tilde{\tau}^{m}} w_{\tilde{\tau}}, h d i v v_{\tilde{\tau}}\right)-\left(2 s^{\prime}+m\right)\left(\frac{h \gamma}{\tilde{\tau}^{1+m}} \nabla w, h v_{\tilde{\tau}}\right)\right) \\
& \leq \eta_{3} h^{2}\left\|\frac{\nabla w}{\tilde{\tau}^{s^{\prime}+m+2}}\right\|_{2}^{2}+\frac{C}{\eta_{3}}\left\|h \tilde{\tau}^{s^{\prime}} \nabla v_{\tilde{\tau}}\right\|_{2}^{2}+C\left\|h \tilde{\tau}^{s^{\prime}} \nabla v\right\|_{2}^{2}+C\left\|h w_{\tilde{\tau}}\right\|_{2}^{2}+C\left(\gamma h f_{\tilde{\tau}}, h^{2} w_{\tilde{\tau} \tilde{\tau}}\right) \\
& \quad+C\left(\tilde{\tau}^{s s^{\prime}} h g_{\tilde{\tau}}, h^{2} v_{\tilde{\tau} \tilde{\tau}}\right) .
\end{aligned}
$$

In addition,

$$
\begin{aligned}
& \frac{\mu}{2}\left\|\tilde{\tau}^{s^{\prime}} h \nabla v_{\tilde{\tau}}\right\|_{2}^{2}+\frac{\mu+\lambda}{2}\left\|h \tilde{\tau}^{s^{\prime}} d i v v_{\tilde{\tau}}\right\|_{2}^{2}-\left(\frac{h \gamma}{\tilde{\tau}^{m}} w_{\tilde{\tau}}, h d i v v_{\tilde{\tau}}\right)-\left(2 s^{\prime}+m\right)\left(\frac{h \gamma}{\tilde{\tau}^{1+m}} \nabla w, h v_{\tilde{\tau}}\right) \\
\geq & \frac{\mu}{4}\left\|\tilde{\tau}^{s^{\prime}} h \nabla v_{\tilde{\tau}}\right\|_{2}^{2}-\left(\frac{2 \gamma^{2}}{\mu}\left\|h w_{\tilde{\tau}}\right\|_{2}^{2}+\frac{2\left(2 s^{\prime}+1\right)^{2} \gamma^{2}}{\mu}\|h w\|_{2}^{2}\right) .
\end{aligned}
$$


Thus, it follows from $\int_{\tilde{\tau}_{0}}^{t}(2.33) d \tilde{\tau}+\frac{2 \gamma^{2}}{\mu} \times(2.21)+\frac{2\left(2 s^{\prime}+1\right)^{2} \gamma^{2}}{\mu} \times(2.12)$ together with (2.34) that

$$
\begin{aligned}
& \quad h\left\|\tilde{\tau}^{s^{\prime}} h \nabla v_{\tilde{\tau}}\right\|_{2}^{2}+\int_{\tilde{\tau}_{0}}^{t}\left(\left\|h^{2} w_{\tilde{\tau} \tilde{\tau}}\right\|_{2}^{2}+\left\|h^{2} \tilde{\tau}^{s^{\prime}} v_{\tilde{\tau} \tilde{\tau}}\right\|_{2}^{2}\right) d \tilde{\tau} \\
& \leq C h\left\|v_{\tilde{\tau}}\left(\tilde{\tau}_{0}\right)\right\|_{H^{1}}^{2}+C\left(\eta_{2}\right) h\left\|\left(w_{0}, v_{0}\right)\right\|_{H^{2}}^{2}+C \eta_{2} h^{2} \int_{\tilde{\tau}_{0}}^{t}\left\|\frac{\nabla w}{\tilde{\tau}^{s^{\prime}+m+1}}\right\|_{2}^{2} d \tilde{\tau} \\
& \quad+C\left(\eta_{2}\right) \int_{\tilde{\tau}_{0}}^{t}\left(A_{1}+A_{2}+A_{3}\right) d \tilde{\tau}+\eta_{3} h^{2} \int_{\tilde{\tau}_{0}}^{t}\left\|\frac{\nabla w}{\tilde{\tau}^{s^{\prime}+m+2}}\right\|_{2}^{2} d \tilde{\tau}+\frac{C}{\eta_{3}} \int_{\tilde{\tau}_{0}}^{t}\left\|h \tilde{\tau}^{s^{\prime}} \nabla v_{\tilde{\tau}}\right\|_{2}^{2} d \tilde{\tau} \\
& \quad+C \int_{\tilde{\tau}_{0}}^{t}\left(\left\|h \tilde{\tau}^{s^{\prime}} \nabla v\right\|_{2}^{2}+\left\|h w_{\tilde{\tau}}\right\|_{2}^{2}+\left(\gamma h f_{\tilde{\tau}}, h^{2} w_{\tilde{\tau} \tilde{\tau}}\right)+\left(\tilde{\tau}^{s s^{\prime}} h g_{\tilde{\tau}}, h^{2} v_{\tilde{\tau} \tilde{\tau}}\right)\right) d \tilde{\tau} .
\end{aligned}
$$

Utilizing the estimates of $\int_{\tilde{\tau}_{0}}^{t}\left\|h \tilde{\tau}^{s^{\prime}} \nabla v_{\tilde{\tau}}\right\|_{2}^{2} d \tilde{\tau}, \int_{\tilde{\tau}_{0}}^{t}\left(\left\|h \tilde{\tau}^{s^{\prime}} \nabla v\right\|_{2}^{2}+\left\|h w_{\tilde{\tau}}\right\|_{2}^{2}\right) d \tilde{\tau}$ in Lemmas 2.12.3, and setting $\eta_{2}=\eta_{3}^{2}$, we then complete the proof of (2.27) from (2.35).

To derive the higher order energy estimates of $(w, v)$, as in [19], we will treat the interior energy estimates and the boundary energy estimates of $(w, v)$ separately.

Lemma 2.5 (Weighted interior energy estimates of $(w, v)$ ). For $\delta \in(0,1)$, define $B_{\delta}=\{y$ : $|y|<\delta\}$. Choosing the function $\chi_{0}(y) \in C_{0}^{\infty}\left(B_{\delta}\right)$, then we have that for $t \geq \tilde{\tau}_{0}$, and $k=1,2,3$,

$$
\begin{aligned}
& \quad h\left\|\chi_{0} D^{k} w\right\|_{2}^{2}+h\left\|\tilde{\tau}^{s^{\prime}} \chi_{0} D^{k} v\right\|_{2}^{2}+\int_{\tilde{\tau}_{0}}^{t}\left(\left\|\tilde{\tau}^{s^{\prime}} \chi_{0} \nabla D^{k} v\right\|_{2}^{2}+\left\|\frac{\chi_{0} D^{k} w}{\tilde{\tau}^{s^{\prime}+m}}\right\|_{2}^{2}\right) d \tau \\
& \leq C \int_{\tilde{\tau}_{0}}^{t}\left(\left\|\tilde{\tau}^{s^{\prime}} D^{k-1} v\right\|_{H^{1}}^{2}+\left|\left(\chi_{0}^{2} D^{k} f, D^{k} w\right)\right|+\left\|\tilde{\tau}^{s^{\prime}} D^{k-1} g\right\|_{2}^{2}+\left\|h \tilde{\tau}^{s^{\prime}} D^{k-1} v_{\tau}\right\|_{2}^{2}\right) d \tau \\
& \quad+C\left\|\left(w_{0}, v_{0}\right)\right\|_{H^{k}}^{2} .
\end{aligned}
$$

Proof. Computing $\int_{S_{0}} \chi_{0}^{2}(y) \gamma D^{k}(2.5 \mathrm{a}) \times D^{k} w d y$ and $\int_{S_{0}} \chi_{0}^{2} \tilde{\tau}^{2 s^{\prime}} D^{k}(2.5 \mathrm{~b}) \cdot D^{k} v d y$ yield respectively

$$
\frac{1}{2}(1+\alpha) h \gamma \frac{d}{d \tilde{\tau}}\left\|\chi_{0} D^{k} w\right\|_{2}^{2}+\frac{\gamma}{\tilde{\tau}^{m}}\left(\chi_{0}^{2} d i v D^{k} v, D^{k} w\right)=\left(\gamma \chi_{0}^{2} D^{k} f, D^{k} w\right)
$$

and

$$
\begin{aligned}
& \frac{1}{2}(1+\alpha) h \frac{d}{d \tilde{\tau}^{\prime}}\left\|\tilde{\tau}^{s^{\prime}} \chi_{0} D^{k} v\right\|_{2}^{2}+\left(\alpha-s^{\prime}(1+\alpha)\right) h\left\|\tilde{\tau}^{s^{\prime}-\frac{1}{2}} \chi_{0} D^{k} v\right\|_{2}^{2}-\left(\chi_{0}^{2} \tilde{\tau}^{2 s^{\prime}} L D^{k} v, D^{k} v\right) \\
& +\left(\frac{\gamma}{\tilde{\tau}^{m}} \chi_{0}^{2} \nabla D^{k} w, D^{k} v\right)=\left(\chi_{0}^{2} \tilde{\tau}^{2 s^{\prime}} D^{k} g, D^{k} v\right) .
\end{aligned}
$$

Note that

$$
\begin{aligned}
& -\left(\chi_{0}^{2} \tilde{\tau}^{2 s^{\prime}} L D^{k} v, D^{k} v\right) \\
= & \mu\left(\tilde{\tau}^{2 s^{\prime}} \nabla D^{k} v, \nabla\left(\chi_{0}^{2} D^{k} v\right)\right)+(\mu+\lambda)\left(\tilde{\tau}^{2 s^{\prime}} \operatorname{div}\left(D^{k} v\right), \operatorname{div}\left(\chi_{0}^{2} D^{k_{v}}\right)\right) \\
\geq & \frac{\mu}{2}\left\|\chi_{0} \tilde{\tau}^{s^{\prime}} \nabla D^{k} v\right\|_{2}^{2}-C\left\|\tilde{\tau}^{s^{\prime}} D^{k} v\right\|_{2}^{2}
\end{aligned}
$$


and for small $\eta_{4}>0$,

$$
\begin{aligned}
& \frac{\gamma}{\tilde{\tau}^{m}}\left(\chi_{0}^{2} d i v D^{k} v, D^{k} w\right)+\left(\frac{\gamma}{\tilde{\tau}^{m}} \chi_{0}^{2} \nabla D^{k} w, D^{k} v\right) \\
= & -\frac{\gamma}{\tilde{\tau}^{m}}\left(D^{k} w, \nabla \chi_{0}^{2} \cdot D^{k} v\right) \\
\leq & \eta_{4}\left\|\frac{\chi_{0} D^{k} w}{\tilde{\tau}^{s^{\prime}+m}}\right\|_{2}^{2}+\frac{C}{\eta_{4}}\left\|\tilde{\tau}^{s^{\prime}} D^{k} v\right\|_{2}^{2} .
\end{aligned}
$$

Adding (2.37) to (2.38) and then applying (2.39)-(2.40), we arrive at

$$
\begin{aligned}
& \frac{1+\alpha}{2} \frac{d}{d \tau}\left(h \gamma\left\|\chi_{0} D^{k} w\right\|_{2}^{2}+h\left\|\tilde{\tau}^{s^{\prime}} \chi_{0} D^{k} v\right\|_{2}^{2}\right)+\frac{\mu}{2}\left\|\tilde{\tau}^{s^{\prime}} \chi_{0} \nabla D^{k} v\right\|_{2}^{2} \\
\leq & \eta_{4}\left\|\frac{\chi_{0} D^{k} w}{\tilde{\tau}^{s+m}}\right\|_{2}^{2}+\frac{C}{\eta_{4}}\left\|\tilde{\tau}^{s^{\prime}} D^{k} v\right\|_{2}^{2}+\left(\gamma \chi_{0}^{2} D^{k} f, D^{k} w\right)+\left(\chi_{0}^{2} \tilde{\tau}^{2 s^{\prime}} D^{k} g, D^{k} v\right) .
\end{aligned}
$$

Since

$$
\begin{aligned}
& \left(\chi_{0}^{2} \tilde{\tau}^{2 s^{\prime}} D^{k} g, D^{k} v\right)=-\tilde{\tau}^{2 s^{\prime}}\left(D^{k-1} g, D\left(\chi_{0}^{2} D^{k} v\right)\right) \\
= & -\tilde{\tau}^{2 s^{\prime}}\left(D^{k-1} g, D\left(\chi_{0}^{2}\right) D^{k} v\right)-\tilde{\tau}^{2 s^{\prime}}\left(D^{k-1} g, \chi_{0}^{2} D^{k+1} v\right),
\end{aligned}
$$

we have

$$
\left|\left(\chi_{0}^{2} \tilde{\tau}^{2 s^{\prime}} D^{k} g, D^{k} v\right)\right| \leq \frac{\mu}{4}\left\|\tilde{\tau}^{s^{\prime}} \chi_{0} D^{k+1} v\right\|_{2}^{2}+C\left\|\tilde{\tau}^{s^{\prime}} D^{k-1} g\right\|_{2}^{2}
$$

Then substituting (2.42) into (2.41) yields

$$
\begin{aligned}
& \frac{1+\alpha}{2} \frac{d}{d \tilde{\tau}}\left(h \gamma\left\|\chi_{0} D^{k} w\right\|_{2}^{2}+h\left\|\tilde{\tau}^{s^{\prime}} \chi_{0} D^{k} v\right\|_{2}^{2}\right)+\frac{\mu}{4}\left\|\tilde{\tau}^{s^{\prime}} \chi_{0} \nabla D^{k} v\right\|_{2}^{2} \\
\leq & \eta_{4}\left\|\frac{\chi_{0} D^{k} w}{\tilde{\tau}^{s+m}}\right\|_{2}^{2}+\frac{C}{\eta_{4}}\left\|\tilde{\tau}^{s^{\prime}} D^{k} v\right\|_{2}^{2}+\left(\gamma \chi_{0}^{2} D^{k} f, D^{k} w\right)+C\left\|\tilde{\tau}^{s^{\prime}} D^{k-1} g\right\|_{2}^{2} .
\end{aligned}
$$

In addition, it follows from $\frac{1}{\tilde{\tau}^{m}} \times\left\{(2 \mu+\lambda) \tilde{\tau}^{m} \times \nabla(3.1)+(3.2)\right\}$ and direct computation that

$$
\begin{aligned}
& (1+\alpha)(2 \mu+\lambda) h \partial_{\tilde{\tau}}(\nabla w)+\frac{\gamma \nabla w}{\tilde{\tau}^{2 s^{\prime}+2 m}} \\
= & -\frac{\mu}{\tilde{\tau}^{m}}(\nabla d i v u-\Delta u)+(2 \mu+\lambda) \nabla f \\
& -\frac{1}{\tilde{\tau}^{m}}\left((1+\alpha) h \partial_{\tilde{\tau}} v+\frac{\alpha h}{\tilde{\tau}} v\right)+\frac{1}{\tilde{\tau}^{m}} g .
\end{aligned}
$$


And computing $\int_{S_{0}} \chi_{0}^{2} D^{k}(2.44) \cdot \nabla D^{k} w$ yields

$$
\begin{aligned}
& \quad \frac{1+\alpha}{2}(2 \mu+\lambda) h \frac{d}{d \tilde{\tau}}\left\|\chi_{0} \nabla D^{k} w\right\|_{2}^{2}+\gamma\left\|\frac{\chi_{0} \nabla D^{k} w}{\tilde{\tau}^{s^{\prime}+m}}\right\|_{2}^{2} \\
& \leq \frac{\mu}{\tilde{\tau}^{m}}\left|\left(\chi_{0}^{2} D^{k}(\nabla d i v v-\Delta v), \nabla D^{k} w\right)\right| \\
& \quad+C\left|\left((2 \mu+\lambda) \chi_{0} \nabla D^{k} f-\frac{\chi_{0}}{\tilde{\tau}^{m}}\left(h D^{k} v_{\tau}+\frac{h D^{k} v}{\tilde{\tau}}-D^{k} g\right), \chi_{0} \nabla D^{k} w\right)\right| \\
& \leq \frac{\gamma}{2}\left\|\frac{\chi_{0} \nabla D^{k} w}{\tilde{\tau}^{s^{\prime}+m}}\right\|_{2}^{2}+C\left(\left|\chi_{0}^{2} D^{k+1} f, D^{k+1} w\right|+\left\|h \tilde{\tau}^{s^{\prime}} D^{k_{\tau}}\right\|_{2}^{2}+\left\|\tilde{\tau}^{s^{\prime}} D^{k} v\right\|_{H^{1}}^{2}\right. \\
& \left.\quad+\left\|\tilde{\tau}^{s^{\prime}} D^{k} g\right\|_{2}^{2}\right),
\end{aligned}
$$

here we have used the following fact

$$
\begin{aligned}
& \left(\chi_{0}^{2} D^{k} \Delta v, \nabla D^{k} w\right)=-\sum_{i, j}\left(\partial_{j} D^{k} v_{i}, \partial_{j}\left(\chi_{0}^{2} \partial_{i} D^{k} w\right)\right) \\
= & \left.\left.-\sum_{i, j}\left(\partial_{j} D^{k} v_{i}, \partial_{j}\left(\chi_{0}^{2}\right) \partial_{i} D^{k} w\right)+\sum_{i, j}\left(\partial_{i}\left(\chi_{0}^{2}\right) \partial_{j} D^{k} v_{i}, \partial_{j} D^{k} w\right)\right)+\sum_{i, j}\left(\chi_{0}^{2} \partial_{i} \partial_{j} D^{k} v_{i}, \partial_{j} D^{k} w\right)\right) \\
= & \left.\left.-\sum_{i, j}\left(\partial_{j} D^{k} v_{i}, \partial_{j}\left(\chi_{0}^{2}\right) \partial_{i} D^{k} w\right)+\sum_{i, j}\left(\partial_{i}\left(\chi_{0}^{2}\right) \partial_{j} D^{k} v_{i}, \partial_{j} D^{k} w\right)\right)+\left(\chi_{0}^{2} \nabla d i v D^{k} v, \nabla D^{k} w\right)\right)
\end{aligned}
$$

to derive

$$
\frac{1}{\tilde{\tau}^{m}}\left|\left(\chi_{0}^{2} D^{k}(\nabla d i v v-\Delta v), \nabla D^{k} w\right)\right| \leq \frac{\gamma}{8}\left\|\frac{\chi_{0} \nabla D^{k} w}{\tilde{\tau}^{s^{\prime}}+m}\right\|_{2}^{2}+C\left\|\tilde{\tau}^{s^{\prime}} D^{k} v\right\|_{H^{1}}^{2} .
$$

From (2.45), we have that for $k=1,2,3$,

$$
\begin{aligned}
& h \frac{d}{d \tau}\left\|\chi_{0} D^{k} w\right\|_{2}^{2}+\left\|\frac{\chi_{0} D^{k} w}{\tilde{\tau}^{s^{\prime}+m}}\right\|_{2}^{2} \\
\leq & C\left(\left|\left(\chi_{0}^{2} D^{k} f, D^{k} w\right)\right|+\left\|h \tilde{\tau}^{s^{\prime}} D^{k-1} v_{\tau}\right\|_{2}^{2}+\left\|\tilde{\tau}^{s^{\prime}} D^{k-1} v\right\|_{H^{1}}^{2}+\left\|\tilde{\tau}^{s^{\prime}} D^{k-1} g\right\|_{2}^{2}\right) .
\end{aligned}
$$

Adding (2.43) to (2.46) and choosing $\eta_{4}=\frac{1}{2}$ yield

$$
\begin{aligned}
& h \frac{d}{d \tau}\left(\gamma\left\|\chi_{0} D^{k} w\right\|_{2}^{2}+\left\|\tilde{\tau}^{s^{\prime}} \chi_{0} D^{k} v\right\|_{2}^{2}\right)+\left\|\tilde{\tau}^{s^{\prime}} \chi_{0} \nabla D^{k} v\right\|_{2}^{2}+\left\|\frac{\chi_{0} D^{k} w}{\tilde{\tau}^{s^{\prime}+m}}\right\|_{2}^{2} \\
\leq & C\left(\left\|\tilde{\tau}^{s^{\prime}} D^{k-1} v\right\|_{H^{1}}^{2}+\left|\left(\chi_{0}^{2} D^{k} f, D^{k} w\right)\right|+\left\|\tilde{\tau}^{s^{\prime}} D^{k-1} g\right\|_{2}^{2}+\left\|h \tilde{\tau}^{s} D^{k-1} v_{\tau}\right\|_{2}^{2}\right) .
\end{aligned}
$$

Integrating (2.47) with respect to the variable $\tau$ over $\left(\tilde{\tau}_{0}, t\right)$ derives (2.36).

Next we treat the weighted energy estimates of $(w, v)$ near the boundary $[0, T] \times \partial S_{0}$. To this end, it is convenient to use the following spherical coordinate transformation

$$
\left\{\begin{array}{l}
y_{1}=r \cos \theta, \\
y_{2}=r \sin \theta \cos \varphi, \\
y_{3}=r \sin \theta \sin \varphi,
\end{array}\right.
$$


and decompose $v=\left(v_{1}, v_{2}, v_{3}\right)$ as

$$
\left\{\begin{array}{l}
v_{r}=\cos \theta v_{1}+\sin \theta \cos \varphi v_{2}+\sin \theta \sin \varphi v_{3} \\
v_{\theta}=-\sin \theta v_{1}+\cos \theta \cos \varphi v_{2}+\cos \theta \sin \varphi v_{3} \\
v_{\varphi}=-\sin \varphi v_{2}+\cos \varphi v_{3}
\end{array}\right.
$$

where $\varphi \in[0,2 \pi]$ and $\theta \in[0, \pi]$. Set $V=\left(v_{r}, v_{\theta}, v_{\varphi}\right)^{T}$ and $V_{\Gamma}=\left(v_{\theta}, v_{\varphi}\right)^{T}$, and denote

$$
\begin{aligned}
& \overline{\operatorname{div} V}=\partial_{r} v_{r}+\frac{1}{r} \partial_{\theta} v_{\theta}+\frac{1}{r \sin \theta} \partial_{\varphi} v_{\varphi}, \\
& \bar{\Delta}=\partial_{r}^{2}+\frac{1}{r^{2}} \partial_{\theta}^{2}+\frac{1}{r^{2} \sin ^{2} \theta} \partial_{\varphi}^{2}, \\
& \bar{\nabla}^{\prime}=\left(\frac{1}{r} \partial_{\theta}, \frac{1}{r \sin \theta} \partial_{\varphi}\right)^{T}, \quad g_{\Gamma}=\left(g_{\theta}, g_{\varphi}\right)^{T},
\end{aligned}
$$

$\overline{\nabla d i v}$ as the form of $\nabla$ div in the spherical coordinates.

In addition, we denote $L(V, k)$ and $L\left(\partial_{\Gamma}^{k} V\right)$ by the linear combinations of $D_{x}^{l} V(l \leq k)$ and $\partial_{\Gamma}^{l} V(l \leq k)$ with the smooth function coefficients respectively, where $\partial_{\Gamma}\left(=\partial_{\varphi}\right.$ or $\left.\partial_{\theta}\right)$ is the vector field tangent to $\partial S_{0}$. Then it follows from direct computation that the Eqs. (2.5a)(2.5b) have such forms:

$$
\begin{aligned}
& (1+\alpha) h w_{\tilde{\tau}}+\frac{1}{\tilde{\tau}^{m}} \overline{\operatorname{div}} V=f+\frac{1}{\tilde{\tau}^{m}} L(V, 0), \\
& (1+\alpha) h \partial_{\tilde{\tau} v_{r}}+\frac{\alpha h}{\tilde{\tau}} v_{r}-\mu \bar{\Delta} v_{r}-(\mu+\lambda) \partial_{r} \overline{\operatorname{div}} V+\frac{\gamma}{\tilde{\tau}^{2 s^{\prime}+m}} \partial_{r} w=g_{r}+L(V, 1), \\
& (1+\alpha) h \partial_{\tilde{\tau}} V_{\Gamma}+\frac{\alpha h}{\tilde{\tau}} V_{\Gamma}-\mu \bar{\Delta} V_{\Gamma}-(\mu+\lambda) \bar{\nabla}^{\prime} \overline{\operatorname{div}} V+\frac{\gamma}{\tilde{\tau}^{2 s^{\prime}+m}} \bar{\nabla}^{\prime} w=g_{\Gamma}+L(V, 1) .
\end{aligned}
$$

Set $\mathcal{O}=\left\{y: \frac{3}{4}<r \leq 1, \varphi \in[0,2 \pi], \theta \in[\pi / 4,3 \pi / 4]\right\}$. We choose a function $\chi_{1}(y) \in C_{0}^{\infty}$ such that $\operatorname{supp} \chi_{1}(y) \subset \mathcal{O}$ and $\chi_{1}(y)=1$ on $\partial S_{0}$. Here we point out that the other left domain in $\left\{y: \frac{3}{4}<r \leq 1, \varphi \in[0,2 \pi], \theta \in[0, \pi]\right\}$ can be successively changed into $\mathcal{O}$ by the coordinate rotations. Thus, it does not lose the generality, we only consider the partial boundary domain $\mathcal{O}$ instead of the whole boundary domain for (2.48a)-(2.48c) (one can also see some details in (4.39)-(4.42) of [13]).

Let $\partial_{\Gamma}^{k}=\partial_{\theta}^{k_{1}} \partial_{\varphi}^{k_{2}}$ with $k_{1}+k_{2}=k$. Then one can easily verify that

$$
\left\{\begin{array}{l}
{\left[\partial_{\Gamma}^{k}, \overline{d i v}\right] V=L\left(\partial_{\Gamma}^{k} V, 0\right), \quad\left[\partial_{\Gamma}^{k}, \bar{\Delta}\right] V=L\left(\partial_{\Gamma}^{k+1} V, 0\right),} \\
{\left[\partial_{\Gamma}^{k}, \partial_{r} \overline{d i v}\right] V=L\left(\partial_{\Gamma}^{k} V, 1\right), \quad\left[\partial_{\Gamma}^{k}, \bar{\nabla}^{\prime} \overline{d i v}\right] V=L\left(\partial_{\Gamma}^{k+1} V, 0\right),} \\
{\left[\partial_{\Gamma}^{k}, \bar{\nabla}^{\prime}\right] w=L\left(\partial_{\Gamma}^{k} w, 0\right) .}
\end{array}\right.
$$

Based on the preparations above, we now establish the tangent energy estimates of $(w, v)$. 
Lemma 2.6 (Weighted tangent energy estimates of $(w, v)$ ). For $t \geq \tilde{\tau}_{0}, 0<\eta<1$, and $k=1,2,3$, we have

$$
\begin{aligned}
& h\left\|\chi_{1} \partial_{\Gamma}^{k} w\right\|_{2}^{2}+h\left\|\tilde{\tau}^{s^{\prime}} \chi_{1} \partial_{\Gamma}^{k} V\right\|_{2}^{2}+\int_{\tilde{\tau}_{0}}^{t}\left\|\tilde{\tau}^{s^{\prime}} \chi_{1} \bar{\nabla}\left(\partial_{\Gamma}^{k} V\right)\right\|_{2}^{2} d \tilde{\tau} \\
& \leq C(\eta) \int_{\tilde{\tau}_{0}}^{t}\left(\left\|\tilde{\tau}^{s^{\prime}} L\left(\partial_{\Gamma}^{k} V, 0\right)\right\|_{2}^{2}+\left|\left(\partial_{\Gamma}^{k} f, \chi_{1}^{2} \partial_{\Gamma}^{k} w\right)\right|+\left\|\tilde{\tau}^{s^{\prime}} \partial_{\Gamma}^{k-1} g\right\|_{2}^{2}\right) d \tilde{\tau} \\
& \quad+C \eta \int_{\tilde{\tau}_{0}}^{t}\left\|\frac{\partial_{\Gamma}^{k} w}{\tilde{\tau}^{s^{\prime}+m}}\right\|_{2}^{2} d \tilde{\tau}+C\left\|\left(w_{0}, v_{0}\right)\right\|_{H^{k}}^{2} .
\end{aligned}
$$

Proof. Acting $\partial_{\Gamma}^{k}$ on two hand sides of (2.48a)-(2.48c) and applying (2.49), we have

$$
\begin{aligned}
& (1+\alpha) h\left(\partial_{\Gamma}^{k} w\right)_{\tilde{\tau}}+\frac{1}{\tilde{\tau}^{m}} \overline{\operatorname{div}} \partial_{\Gamma}^{k} V=\partial_{\Gamma}^{k} f+\frac{1}{\tilde{\tau}^{m}} L\left(\partial_{\Gamma}^{k} V, 0\right) \\
& (1+\alpha) h \partial_{\tilde{\tau}}\left(\partial_{\Gamma}^{k} v_{r}\right)+\frac{\alpha h}{\tilde{\tau}} \partial_{\Gamma}^{k} v_{r}-\mu \bar{\Delta}\left(\partial_{\Gamma}^{k} v_{r}\right)-(\mu+\lambda) \partial_{r} \overline{\operatorname{div}}\left(\partial_{\Gamma}^{k} V\right)+\frac{\gamma}{\tilde{\tau}^{2 s^{\prime}+m}} \partial_{r}\left(\partial_{\Gamma}^{k} w\right) \\
& =\partial_{\Gamma}^{k} g_{r}+L\left(\partial_{\Gamma}^{k} V, 1\right) \\
& (1+\alpha) h \partial_{\tilde{\tau}}\left(\partial_{\Gamma}^{k} V_{\Gamma}\right)+\frac{\alpha h}{\tilde{\tau}} \partial_{\Gamma}^{k} V_{\Gamma}-\mu \bar{\Delta}\left(\partial_{\Gamma}^{k} V_{\Gamma}\right)-(\mu+\lambda) \bar{\nabla}^{\prime} \overline{\operatorname{div}}\left(\partial_{\Gamma}^{k} V\right)+\frac{\gamma}{\tilde{\tau}^{2 s^{\prime}+m}} \bar{\nabla}^{\prime}\left(\partial_{\Gamma}^{k} w\right) \\
& =\partial_{\Gamma}^{k} g_{\Gamma}+L\left(\partial_{\Gamma}^{k} V, 1\right)
\end{aligned}
$$

It follows from $\int_{S_{0}} \gamma \chi_{1}^{2}(2.51 \mathrm{a}) \times \partial_{\Gamma}^{k} w d y$ that

$$
\begin{aligned}
& \frac{1+\alpha}{2} h \frac{d}{d \tilde{\tau}}\left(\gamma\left\|\chi_{1} \partial_{\Gamma}^{k} w\right\|_{2}^{2}\right)+\left(\frac{\gamma}{\tilde{\tau}^{m}} \overline{\operatorname{div}} \partial_{\Gamma}^{k} v, \chi_{1}^{2} \partial_{\Gamma}^{k} w\right) \\
= & \left(\gamma \partial_{\Gamma}^{k} f, \chi_{1}^{2} \partial_{\Gamma}^{k} w\right)+\left(\frac{1}{\tilde{\tau}^{m}} \chi_{1}^{2} L\left(\partial_{\Gamma}^{k} V, 0\right), \partial_{\Gamma}^{k} w\right) .
\end{aligned}
$$

In addition, computing $\int_{S_{0}} \tilde{\tau}^{2 s} \chi_{1}^{2}(2.51 \mathrm{~b}) \times \partial_{\Gamma}^{k} v_{r} d y$ yields

$$
\begin{aligned}
& \frac{1+\alpha}{2} h \frac{d}{d \tilde{\tau}}\left(\left\|\tilde{\tau}^{s^{\prime}} \chi_{1} \partial_{\Gamma}^{k} v_{r}\right\|_{2}^{2}\right)+\left(\alpha-(1+\alpha) s^{\prime}\right) h\left\|\chi_{1} \tilde{\tau}^{s^{\prime}-\frac{1}{2}} \partial_{\Gamma}^{k} v_{r}\right\|_{2}^{2} \\
& \quad-\left(\chi_{1}^{2} \tilde{\tau}^{2 s^{\prime}}\left(\mu \bar{\Delta}\left(\partial_{\Gamma}^{k} v_{r}\right)+(\mu+\lambda) \partial_{r} \overline{d i v}\left(\partial_{\Gamma}^{k} V\right)\right), \partial_{\Gamma}^{k} v_{r}\right)+\left(\chi_{1}^{2} \frac{\gamma}{\tilde{\tau}^{m}} \partial_{r}\left(\partial_{\Gamma}^{k} w\right), \partial_{\Gamma}^{k} v_{r}\right) \\
= & \left(\tilde{\tau}^{2 s^{\prime}} \chi_{1}^{2}\left(\partial_{\Gamma}^{k} g_{r}+L\left(\partial_{\Gamma}^{k} V, 1\right)\right), \partial_{\Gamma}^{k} v_{r}\right),
\end{aligned}
$$

and computing $\int_{S_{0}} \tilde{\tau}^{2 s^{\prime}} \chi_{1}^{2}(2.51 \mathrm{c}) \cdot \partial_{\Gamma}^{k} V_{\Gamma} d y$ yields

$$
\begin{aligned}
& \frac{1+\alpha}{2} h \frac{d}{d \tilde{\tau}}\left(\left\|\tilde{\tau}^{s^{\prime}} \chi_{1} \partial_{\Gamma}^{k} V_{\Gamma}\right\|_{2}^{2}\right)+\left(\alpha-(1+\alpha) s^{\prime}\right) h\left\|\chi_{1} \tilde{\tau}^{s^{\prime}-\frac{1}{2}} \partial_{\Gamma}^{k} V_{\Gamma}\right\|_{2}^{2} \\
& \quad-\left(\chi_{1}^{2} \tilde{\tau}^{2 s^{\prime}}\left(\mu \bar{\Delta}\left(\partial_{\Gamma}^{k} V_{\Gamma}\right)+(\mu+\lambda) \bar{\nabla}^{\prime} \overline{d i v}\left(\partial_{\Gamma}^{k} V\right)\right), \partial_{\Gamma}^{k} V_{\Gamma}\right)+\left(\chi_{1}^{2} \frac{\gamma}{\tilde{\tau}^{m}} \bar{\nabla}^{\prime}\left(\partial_{\Gamma}^{k} w\right), \partial_{\Gamma}^{k} V_{\Gamma}\right) \\
= & \left(\tilde{\tau}^{2 s^{\prime}} \chi_{1}^{2}\left(\partial_{\Gamma}^{k} g_{\Gamma}+L\left(\partial_{\Gamma}^{k} V, 1\right)\right), \partial_{\Gamma}^{k} V_{\Gamma}\right) .
\end{aligned}
$$


Notice that

$$
\begin{aligned}
& -\left(\chi_{1}^{2} \tilde{\tau}^{2 s^{\prime}}\left(\mu \bar{\Delta}\left(\partial_{\Gamma}^{k} v_{r}\right)+(\mu+\lambda) \partial_{r} \overline{\operatorname{div}}\left(\partial_{\Gamma}^{k} V\right)\right), \partial_{\Gamma}^{k} v_{r}\right) \\
& =\mu\left\|\tilde{\tau}^{s^{\prime}} \chi_{1} \bar{\nabla}\left(\partial_{\Gamma}^{k} v_{r}\right)\right\|_{2}^{2}+\mu\left(\tilde{\tau}^{2 s^{\prime}} \bar{\nabla}\left(\partial_{\Gamma}^{k} v_{r}\right), \bar{\nabla}\left(\chi_{1}^{2}\right) \partial_{\Gamma}^{k} v_{r}\right) \\
& +(\mu+\lambda)\left(\tilde{\tau}^{2 s^{\prime}} \overline{\operatorname{div}}\left(\partial_{\Gamma}^{k} V\right), \partial_{r}\left(\chi_{1}^{2} \partial_{\Gamma}^{k} v_{r}\right)\right)
\end{aligned}
$$

and

$$
\begin{aligned}
- & \left(\chi_{1}^{2} \tilde{\tau}^{2 s^{\prime}}\left(\mu \bar{\Delta}\left(\partial_{\Gamma}^{k} V_{\Gamma}\right)+(\mu+\lambda) \bar{\nabla}^{\prime} \overline{\operatorname{div}}\left(\partial_{\Gamma}^{k} V\right)\right), \partial_{\Gamma}^{k} V_{\Gamma}\right) \\
=\mu \| \tilde{\tau}^{s^{\prime}} & \chi_{1} \bar{\nabla}\left(\partial_{\Gamma}^{k} V_{\Gamma}\right) \|_{2}^{2}+\mu\left(\tilde{\tau}^{2 s^{\prime}} \bar{\nabla}\left(\partial_{\Gamma}^{k} v_{r}\right), \bar{\nabla}\left(\chi_{1}^{2}\right) \partial_{\Gamma}^{k} V_{\Gamma}\right) \\
& +(\mu+\lambda)\left(\tilde{\tau}^{2 s^{\prime}} \overline{\operatorname{div}}\left(\partial_{\Gamma}^{k} V\right), \bar{\nabla}^{\prime}\left(\chi_{1}^{2} \partial_{\Gamma}^{k} V_{\Gamma}\right)\right) .
\end{aligned}
$$

Adding (2.55) and (2.56) yields

$$
\begin{aligned}
& -\left(\chi_{1}^{2} \tilde{\tau}^{2 s^{\prime}}\left(\mu \bar{\Delta}\left(\partial_{\Gamma}^{k} v_{r}\right)+(\mu+\lambda) \partial_{r} \overline{\operatorname{div}}\left(\partial_{\Gamma}^{k} V\right)\right), \partial_{\Gamma}^{k} v_{r}\right) \\
& -\left(\chi_{1}^{2} \tilde{\tau}^{2 s^{\prime}}\left(\mu \bar{\Delta}\left(\partial_{\Gamma}^{k} v_{r}\right)+(\mu+\lambda) \partial_{r} \overline{\operatorname{div}}\left(\partial_{\Gamma}^{k} V\right)\right), \partial_{\Gamma}^{k} v_{r}\right) \\
\geq & \frac{\mu}{2}\left\|\tilde{\tau}^{s^{\prime}} \chi_{1} \bar{\nabla}\left(\partial_{\Gamma}^{k} V\right)\right\|_{2}^{2}-C\left\|\tilde{\tau}^{s^{\prime}} \partial_{\Gamma}^{k} V\right\|_{2}^{2} .
\end{aligned}
$$

Additionally, for small $\eta>0$, one has

$$
\begin{aligned}
& \left(\frac{\gamma}{\tilde{\tau}^{m}} \overline{\operatorname{div}} \partial_{\Gamma}^{k} v, \chi_{1}^{2} \partial_{\Gamma}^{k} w\right)+\left(\chi_{1}^{2} \frac{\gamma}{\tilde{\tau}^{m}} \partial_{r}\left(\partial_{\Gamma}^{k} w\right), \partial_{\Gamma}^{k} v_{r}\right)+\left(\chi_{1}^{2} \frac{\gamma}{\tilde{\tau}^{m}} \bar{\nabla}^{\prime}\left(\partial_{\Gamma}^{k} w\right), \partial_{\Gamma}^{k} V_{\Gamma}\right) \\
= & -\frac{\gamma}{\tilde{\tau}^{m}}\left(\partial_{\Gamma}^{k} w, \bar{\nabla}\left(\chi_{1}^{2}\right) \cdot \partial_{\Gamma}^{k} V\right) \\
\leq & \eta\left\|\frac{\partial_{\Gamma}^{k} w}{\tilde{\tau}^{s^{\prime}+m}}\right\|_{2}^{2}+C(\eta)\left\|\tilde{\tau}^{s^{\prime}} \partial_{\Gamma}^{k} V\right\|_{2}^{2}
\end{aligned}
$$

and

$$
\begin{aligned}
& \left(\frac{1}{\tilde{\tau}^{m}} \chi_{1}^{2} L\left(\partial_{\Gamma}^{k} V, 0\right), \partial_{\Gamma}^{k} w\right)+\tilde{\tau}^{2 s^{\prime}}\left(\left(\chi_{1}^{2} L\left(\partial_{\Gamma}^{k} V, 1\right), \partial_{\Gamma}^{k} v_{r}\right)+\left(\chi_{1}^{2} L\left(\partial_{\Gamma}^{k} V, 1\right), \partial_{\Gamma}^{k} V_{\Gamma}\right)\right) \\
\leq & \frac{\mu}{4}\left\|\tilde{\tau}^{s^{\prime}} \chi_{1} \bar{\nabla}\left(\partial_{\Gamma}^{k} V\right)\right\|_{2}^{2}+\eta\left\|\frac{\partial_{\Gamma}^{k} w}{\tilde{\tau}^{s^{\prime}+m}}\right\|_{2}^{2}+C(\eta)\left\|\tilde{\tau}^{s^{\prime}} L\left(\partial_{\Gamma}^{k} V, 0\right)\right\|_{2}^{2} .
\end{aligned}
$$

Note that for small $\eta_{5}>0$,

$$
\begin{aligned}
& \left|\left(\chi_{1}^{2} \tilde{\tau}^{2 s^{\prime}} \partial_{\Gamma}^{k} g, \partial_{\Gamma}^{k} V\right)\right| \\
\leq & \left|\left(\tilde{\tau}^{2 s^{\prime}} \partial_{\Gamma}^{k-1} g, \partial_{\Gamma}\left(\chi_{1}^{2}\right) \partial_{\Gamma}^{k} V\right)\right|+\left|\left(\chi_{1}^{2} \tilde{\tau}^{2 s^{\prime}} \partial_{\Gamma}^{k-1} g, \partial_{\Gamma}^{k+1} V\right)\right| \\
\leq & C\left\|\tilde{\tau}^{s^{\prime}} \partial_{\Gamma}^{k-1} g\right\|_{2}^{2}+\eta_{5}\left\|\tilde{\tau}^{s} \chi_{1} \bar{\nabla}\left(\partial_{\Gamma}^{k} V\right)\right\|_{2}^{2}+C\left(\eta_{5}\right)\left\|\tilde{\tau}^{s^{\prime}}\left(\partial_{\Gamma}^{k} V\right)\right\|_{2}^{2} .
\end{aligned}
$$


Adding (2.52), (2.53) and (2.54), we have that by (2.57)-(2.59) and (2.60) with small $\eta>0$,

$$
\begin{aligned}
& \frac{d}{d \tilde{\tau}}\left(h \gamma\left\|\chi_{1} \partial_{\Gamma}^{k} w\right\|_{2}^{2}+h\left\|\tilde{\tau}^{s^{\prime}} \chi_{1} \partial_{\Gamma}^{k} V\right\|_{2}^{2}\right)+\left\|\tilde{\tau}^{s^{\prime}} \chi_{1} \bar{\nabla}\left(\partial_{\Gamma}^{k} V\right)\right\|_{2}^{2} \\
\leq & \eta\left\|\frac{\partial_{\Gamma}^{k} w}{\tilde{\tau}^{s^{\prime}+m}}\right\|_{2}^{2}+C(\eta)\left\|\tilde{\tau}^{s^{\prime}} L\left(\partial_{\Gamma}^{k} V, 0\right)\right\|_{2}^{2}+C\left|\left(\partial_{\Gamma}^{k} f, \chi_{1}^{2} \partial_{\Gamma}^{k} w\right)\right|+C\left\|\tilde{\tau}^{s^{\prime}} \partial_{\Gamma}^{k-1} g\right\|_{2}^{2} .
\end{aligned}
$$

Integrating this on the variable $\tilde{\tau}$ over $\left(\tilde{\tau}_{0}, t\right)$, we can complete the proof of $(2.50)$.

Next we deal with the normal derivative estimates of $w$.

Lemma 2.7 (The first order normal derivative estimate of $\partial_{\Gamma}^{k} w$ ). For $t \geq \tilde{\tau}_{0}, 0<\eta<1$, and $k=0,1,2$, then

$$
\begin{aligned}
& \quad h\left\|\chi_{1} \partial_{r} \partial_{\Gamma}^{k} w\right\|_{2}^{2}+\int_{\tilde{\tau}_{0}}^{t}\left(\left\|\tilde{\tau}^{s^{\prime}} \chi_{1} \overline{\nabla d i v}\left(\partial_{\Gamma}^{k} V\right)\right\|_{2}^{2}+\left\|\chi_{1} \frac{\partial_{r} \partial_{\Gamma}^{k} w}{\tilde{\tau}^{s^{\prime}+m}}\right\|_{2}^{2}\right) d \tilde{\tau} \\
& \leq C\left\|\left(w_{0}, v_{0}\right)\right\|_{H^{k+1}}^{2}+C \eta \int_{\tilde{\tau}_{0}}^{t}\left\|\frac{\partial_{\Gamma}^{k+1} w}{\tilde{\tau}^{s^{\prime}+m}}\right\|_{2}^{2} d \tilde{\tau}+C(\eta) \int_{\tilde{\tau}_{0}}^{t}\left(\left\|\tilde{\tau}^{s^{\prime}} \chi_{1} L\left(\partial_{\Gamma}^{k+1} V, 1\right)\right\|_{2}^{2}\right. \\
& \left.\quad+\left\|h \tilde{\tau}^{s^{\prime}} \chi_{1}\left(\partial_{\Gamma}^{k} V\right)_{\tilde{\tau}}\right\|_{2}^{2}+\left\|\tilde{\tau}^{s^{\prime}} \chi_{1} L\left(\partial_{\Gamma}^{k} V, 1\right)\right\|_{2}^{2}+\left|\left(\chi_{1}^{2} \partial_{\Gamma}^{k} D f, \partial_{\Gamma}^{k} D w\right)\right|+\left\|\tilde{\tau}^{s^{\prime}} \partial_{\Gamma}^{k} g\right\|_{2}^{2}\right) d \tilde{\tau} .
\end{aligned}
$$

Proof. At first, we rewrite $\partial_{r}(2.51 \mathrm{a})$ and $(2.51 \mathrm{~b})$ as

$$
\begin{aligned}
& (1+\alpha) h\left(\partial_{r} \partial_{\Gamma}^{k} w\right)_{\tilde{\tau}}+\frac{1}{\tilde{\tau}^{m}} \partial_{r}^{2} \partial_{\Gamma}^{k} v_{r}=\frac{1}{\tilde{\tau}^{m}} L\left(\partial_{\Gamma}^{k+1} V, 1\right)+\partial_{r} \partial_{\Gamma}^{k} f+\frac{1}{\tilde{\tau}^{m}} L\left(\partial_{\Gamma}^{k} V, 1\right), \\
& (1+\alpha) h \partial_{\tilde{\tau}}\left(\partial_{\Gamma}^{k} v_{r}\right)-(2 \mu+\lambda) \partial_{r}^{2} \partial_{\Gamma}^{k} v_{r}+\frac{\gamma}{\tilde{\tau}^{2 s^{\prime}+m}} \partial_{r}\left(\partial_{\Gamma}^{k} w\right) \\
& =\partial_{\Gamma}^{k} g_{r}+L\left(\partial_{\Gamma}^{k} V, 1\right)-\frac{\alpha h}{\tilde{\tau}^{m}} \partial_{\Gamma}^{k} v_{r}+L\left(\partial_{\Gamma}^{k+2} V, 0\right) .
\end{aligned}
$$

Computing $\frac{(2.62 \mathrm{~b})}{(2 \mu+\lambda) \tilde{\tau}^{m}}+(2.62 \mathrm{a})$ yields

$$
\begin{aligned}
& (1+\alpha) h\left(\partial_{r} \partial_{\Gamma}^{k} w\right)_{\tilde{\tau}}+\frac{\gamma}{2 \mu+\lambda} \frac{\partial_{r} \partial_{\Gamma}^{k} w}{\tilde{\tau}^{2 s^{\prime}+2 m}} \\
= & \frac{1}{\tilde{\tau}^{m}} L\left(\partial_{\Gamma}^{k+1} V, 1\right)+\partial_{r} \partial_{\Gamma}^{k} f+\frac{1}{\tilde{\tau}^{m}} L\left(\partial_{\Gamma}^{k} V, 1\right) \\
& \quad+\frac{1}{(2 \mu+\lambda) \tilde{\tau}^{m}}\left(-(1+\alpha) h \partial_{\tilde{\tau}}\left(\partial_{\Gamma}^{k} v_{r}\right)+\partial_{\Gamma}^{k} g_{r}+L\left(\partial_{\Gamma}^{k} V, 1\right)-\frac{\alpha h}{\tilde{\tau}} \partial_{\Gamma}^{k} v_{r}+L\left(\partial_{\Gamma}^{k+2} V, 0\right)\right) .
\end{aligned}
$$


It follows from $\int_{S_{0}} \chi_{1}^{2}(2.63) \times \partial_{r} \partial_{\Gamma}^{k} w d y$ and direct computation that

$$
\begin{aligned}
& \quad h \frac{d}{d \tilde{\tau}}\left\|\chi_{1} \partial_{r} \partial_{\Gamma}^{k} w\right\|_{2}^{2}+\left\|\chi_{1} \frac{\partial_{r} \partial_{\Gamma}^{k} w}{\tilde{\tau}^{s^{\prime}}+m}\right\|_{2}^{2} \\
& \leq C\left(\left\|\tilde{\tau}^{s} \chi_{1} L\left(\partial_{\Gamma}^{k+1} V, 1\right)\right\|_{2}^{2}+\left\|h \tilde{\tau}^{s^{\prime}} \chi_{1}\left(\partial_{\Gamma}^{k} V\right)_{\tilde{\tau}}\right\|_{2}^{2}\right. \\
& \left.\quad+\left\|\tilde{\tau}^{s^{\prime}} \chi_{1} L\left(\partial_{\Gamma}^{k} V, 1\right)\right\|_{2}^{2}+\left|\left(\chi_{1}^{2} \partial_{r} \partial_{\Gamma}^{k} f, \partial_{r} \partial_{\Gamma}^{k} w\right)\right|+\left\|\tilde{\tau}^{s^{\prime}} \partial_{\Gamma}^{k} g\right\|_{2}^{2}\right) .
\end{aligned}
$$

Integrating $(2.64)$ over $\left(\tilde{\tau}_{0}, t\right)$, we have

$$
\begin{aligned}
& \quad h\left\|\chi_{1} \partial_{r} \partial_{\Gamma}^{k} w\right\|_{2}^{2}+\int_{\tilde{\tau}_{0}}^{t}\left\|\chi_{1} \frac{\partial_{r} \partial_{\Gamma}^{k} w}{\tilde{\tau}^{s^{\prime}+m}}\right\|_{2}^{2} d \tilde{\tau} \\
& \leq C\left\|\left(w_{0}, u_{0}\right)\right\|_{H^{k+1}}^{2}+C \int_{\tilde{\tau}_{0}}^{t}\left(\left\|\tilde{\tau}^{s^{\prime}} \chi_{1} L\left(\partial_{\Gamma}^{k+1} V, 1\right)\right\|_{2}^{2}+\left\|h \tilde{\tau}^{s^{\prime}} \chi_{1}\left(\partial_{\Gamma}^{k} V\right)_{\tilde{\tau}}\right\|_{2}^{2}\right. \\
& \left.\quad+\left\|\tilde{\tau}^{s^{\prime}} \chi_{1} L\left(\partial_{\Gamma}^{k} V, 1\right)\right\|_{2}^{2}+\left|\left(\chi_{1}^{2} \partial_{r} \partial_{\Gamma}^{k} f, \partial_{r} \partial_{\Gamma}^{k} w\right)\right|+\left\|\tilde{\tau}^{s^{\prime}} \partial_{\Gamma}^{k} g\right\|_{2}^{2}\right) d \tilde{\tau} .
\end{aligned}
$$

By (2.65), one has that

$$
\begin{aligned}
-(2 \mu+\lambda) \partial_{r}^{2} \partial_{\Gamma}^{k} v_{r}= & -(1+\alpha) h \partial_{\tilde{\tau}}\left(\partial_{\Gamma}^{k} v_{r}\right)-\frac{\gamma}{\tilde{\tau}^{2 s^{\prime}+m}} \partial_{r}\left(\partial_{\Gamma}^{k} w\right)+\partial_{\Gamma}^{k} g_{r}+L\left(\partial_{\Gamma}^{k} V, 1\right) \\
& -\frac{\alpha h}{\tilde{\tau}} \partial_{\Gamma}^{k} v_{r}+L\left(\partial_{\Gamma}^{k+2} V, 0\right) .
\end{aligned}
$$

Then computing $\int_{S_{0}} \chi_{1}^{2} \tilde{\tau}^{2 s^{\prime}}(2.66) \times \partial_{r}^{2} \partial_{\Gamma}^{k} v_{r} d y$ yields

$$
\begin{aligned}
\left\|\chi_{1} \tilde{\tau}^{s^{\prime}} \partial_{r}^{2} \partial_{\Gamma}^{k} v_{r}\right\|_{2}^{2} \leq & C\left(\left\|h \chi_{1} \tilde{\tau}^{s^{\prime}} \partial_{\tilde{\tau}}\left(\partial_{\Gamma}^{k} v_{r}\right)\right\|_{2}^{2}+\left\|\tilde{\tau}^{s^{\prime}} \partial_{\Gamma}^{k} g_{r}\right\|_{2}^{2}+\left\|\frac{\partial_{r} \partial_{\Gamma}^{k} w}{\tilde{\tau}^{s^{\prime}}+m}\right\|_{2}^{2}\right. \\
& \left.+\left\|\chi_{1} R^{s} L\left(\partial_{\Gamma}^{k} V, 1\right)\right\|_{2}^{2}+\left\|\chi_{1} R^{s} L\left(\partial_{\Gamma}^{k+2} V, 0\right)\right\|_{2}^{2}\right) .
\end{aligned}
$$

On the other hand, by Lemma 2.6, (2.67) and the expression of $\overline{\nabla d i v} V$, we have that for $k=0,1,2$, and small $\eta>0$,

$$
\begin{aligned}
& \int_{\tilde{\tau}_{0}}^{t}\left\|\tilde{\tau}^{s^{\prime}} \chi_{1} \overline{\nabla d i v}\left(\partial_{\Gamma}^{k} V\right)\right\|_{2}^{2} d \tilde{\tau} \\
& \leq C(\eta) \int_{\tilde{\tau}_{0}}^{t}\left(\left\|\chi_{1} \tilde{\tau}^{s^{\prime}} L\left(\partial_{\Gamma}^{k} V, 1\right)\right\|_{2}^{2}+\left|\left(\partial_{\Gamma}^{k+1} f, \chi_{1}^{2} \partial_{\Gamma}^{k+1} w\right)\right|+\left|\left(\chi_{1}^{2} \tilde{\tau}^{2 s^{\prime}} \partial_{\Gamma}^{k+1} g, \partial_{\Gamma}^{k+1} V\right)\right|\right) d \tilde{\tau} \\
& \quad+C(\eta) \int_{\tilde{\tau}_{0}}^{t}\left(\left\|h \chi_{1} \tilde{\tau}^{s} \partial_{\tilde{\tau}}\left(\partial_{\Gamma}^{k} v_{r}\right)\right\|_{2}^{2}+\left\|\tilde{\tau}^{s^{\prime}} \partial_{\Gamma}^{k} g_{r}\right\|_{2}^{2}+\left\|\frac{\partial_{r} \partial_{\Gamma}^{k} w}{\tilde{\tau}^{s^{\prime}+m}}\right\|_{2}^{2}\right. \\
& \left.\quad+\left\|\chi_{1} \tilde{\tau}^{s^{\prime}} L\left(\partial_{\Gamma}^{k+2} V, 0\right)\right\|_{2}^{2}\right) d \tilde{\tau}+C\left\|\left(w_{0}, v_{0}\right)\right\|_{H^{k+1}}^{2}+C \eta \int_{\tilde{\tau}_{0}}^{t}\left\|\frac{\partial_{\Gamma}^{k} D w}{\tilde{\tau}^{s^{\prime}+m}}\right\|_{2}^{2} d \tilde{\tau} .
\end{aligned}
$$


This, together with (2.65), yields

$$
\begin{aligned}
& \quad h\left\|\chi_{1} \partial_{r} \partial_{\Gamma}^{k} w\right\|_{2}^{2}+\int_{\tilde{\tau}_{0}}^{t}\left(\left\|\tilde{\tau}^{s^{\prime}} \chi_{1} \overline{\nabla d i v}\left(\partial_{\Gamma}^{k} V\right)\right\|_{2}^{2}+\left\|\chi_{1} \frac{\partial_{r} \partial_{\Gamma}^{k} w}{\tilde{\tau}^{s^{\prime}+m}}\right\|_{2}^{2}\right) d \tilde{\tau} \\
& \leq C\left\|\left(w_{0}, v_{0}\right)\right\|_{H^{k+1}}^{2}+C \eta \int_{\tilde{\tau}_{0}}^{t}\left\|\frac{\partial_{\Gamma}^{k+1} w}{\tilde{\tau}^{s^{\prime}+m}}\right\|_{2}^{2} d \tilde{\tau}+C(\eta) \int_{\tilde{\tau}_{0}}^{t}\left(\left\|\tilde{\tau}^{s^{\prime}} \chi_{1} L\left(\partial_{\Gamma}^{k+1} V, 1\right)\right\|_{2}^{2}\right. \\
& \left.\quad+\left\|h \tilde{\tau}^{s^{\prime}} \chi_{1}\left(\partial_{\Gamma}^{k} V\right)_{\tilde{\tau}}\right\|_{2}^{2}+\left\|\tilde{\tau}^{s^{\prime}} \chi_{1} L\left(\partial_{\Gamma}^{k} V, 1\right)\right\|_{2}^{2}+\left|\left(\chi_{1}^{2} \partial_{\Gamma}^{k} D f, \partial_{\Gamma}^{k} D w\right)\right|+\left\|\tilde{\tau}^{s^{\prime}} \partial_{\Gamma}^{k} g\right\|_{2}^{2}\right) d \tilde{\tau} .
\end{aligned}
$$

Then the proof of Lemma 2.7 is completed.

As in [19], we now state the following result which can be easily shown by the standard regularity theory on the second order elliptic equation.

Lemma 2.8. For the Stokes equation

$$
\left\{\begin{array}{l}
\operatorname{divu}=f \\
-\Delta u+\nabla P=g \\
u=0 \text { on } \partial S_{0}
\end{array}\right.
$$

we have

$$
\left\|D^{2} u\right\|_{2}^{2}+\|D P\|_{2}^{2} \leq C\left(\|f\|_{H^{1}}^{2}+\|g\|_{2}^{2}\right)
$$

\section{The global energy estimates for problem (1.1a)-(1.1b) with (1.2)}

In this section, based on the results in Section 2, we will establish the global energy estimates of the solution $(w, v)$ to (3.1)-(3.2). For this purpose, we define for $t_{2} \geq t_{1} \geq \tilde{\tau}_{0}$ and $k=2,3$,

$$
\begin{aligned}
& N_{k}\left(t_{1}, t_{2}\right) \\
= & \sup _{t_{1} \leq \tilde{\tau} \leq t_{2}}\left(h\|w\|_{H^{k}}^{2}+h\left\|\tilde{\tau}^{s^{\prime}} v\right\|_{H^{k}}^{2}+h\left\|h w_{\tilde{\tau}}\right\|_{H^{k-1}}^{2}+h\left\|h \tilde{\tau}^{s^{\prime}} v_{\tilde{\tau}}\right\|_{H^{k-2}}^{2}\right) \\
& +\int_{t_{1}}^{t_{2}}\left(\left\|\frac{D w}{\tilde{\tau}^{s^{\prime}+m}}\right\|_{H^{k-1}}^{2}+\left\|h w_{\tilde{\tau}}\right\|_{H^{k-1}}^{2}+\left\|\tilde{\tau}^{s^{\prime}} D v\right\|_{H^{k}}^{2}+\left\|h \tilde{\tau}^{s^{\prime}} D v_{\tilde{\tau}}\right\|_{H^{k-2}}^{2}+\left\|h \tilde{\tau}^{s^{\prime}} v_{\tilde{\tau}}\right\|_{2}^{2}\right) d \tilde{\tau} .
\end{aligned}
$$

To prove Theorem 1.2, we need to obtain the uniform $H^{3}$ estimates of $(w, v)$, i.e., the uniform estimates of $N_{3}\left(\tilde{\tau}_{0}, t\right)$ for any $t \geq \tilde{\tau}_{0}$. As the starting point, we will show

$$
N_{3}\left(\tilde{\tau}_{0}, t\right) \leq C \sup _{\tilde{\tau}_{0} \leq \tilde{\tau} \leq t} h\left\|\tilde{\tau}^{s} g(\tilde{\tau})\right\|_{H^{1}}^{2}+C\left\|\left(w_{0}, v_{0}\right)\right\|_{H^{3}}^{2}+C \int_{\tilde{\tau}_{0}}^{t} M(\tilde{\tau}) d \tilde{\tau}
$$


where

$$
\begin{aligned}
& M(\tilde{\tau}) \\
& =\left|\left(\tilde{\tau}^{2 s^{\prime}} g, h v_{\tilde{\tau}}\right)\right|+\left|\left(\tilde{\tau}^{2 s^{\prime}} h g_{\tilde{\tau}}, h^{2} v_{\tilde{\tau} \tilde{\tau}}\right)\right|+\left|\left(\tilde{\tau}^{2 s^{\prime}} h g_{\tilde{\tau}}, h v_{\tilde{\tau}}\right)\right|+\sum_{k=0}^{3}\left|\left(D^{k} f, D^{k} w\right)\right| \\
& \quad+\sum_{k=0}^{2}\left|\left(\tilde{\tau}^{2 s^{\prime}} D^{k} g, D^{k} v\right)\right|+\left\|\tilde{\tau}^{s^{\prime}+m} f_{0}\right\|_{H^{3}}^{2}+\|f\|_{2}^{2}+\left\|\tilde{\tau}^{s^{\prime}} g\right\|_{H^{2}}^{2}+\left\|\tilde{\tau}^{s^{\prime}} h g_{\tilde{\tau}}\right\|_{2}^{2}+\left\|h f_{\tilde{\tau}}\right\|_{2}^{2},
\end{aligned}
$$

and the definitions of $f, g, f_{0}$ and the number $s^{\prime}$ have been given in Section 2. We will divide the proof procedure of (3.1) into the following four steps.

Step 1. The basic $L^{2}$-energy inequality of $(w, v)$.

By Lemmas 2.1-2.3, we have that for small $\eta>0$ and $t \geq \tilde{\tau}_{0}$,

$$
\begin{aligned}
& \quad h\|w\|_{2}^{2}+h\left\|\tilde{\tau}^{s^{\prime}} v\right\|_{2}^{2}+h\left\|\tilde{\tau}^{s^{\prime}} \nabla v\right\|_{2}^{2}+h\left\|h w_{\tilde{\tau}}\right\|_{2}^{2}+h\left\|h \tilde{\tau}^{s^{\prime}} v_{\tilde{\tau}}\right\|_{2}^{2} \\
& \quad+\int_{\tilde{\tau}_{0}}^{t}\left(\left\|\tilde{\tau}^{s^{\prime}} \nabla v\right\|_{2}^{2}+\left\|\tilde{\tau}^{s^{\prime}+1} \frac{d w}{d \tilde{\tau}}\right\|_{2}^{2}+\left\|h w_{\tilde{\tau}}\right\|_{2}^{2}+\left\|h \tilde{\tau}^{s^{\prime}} v_{\tilde{\tau}}\right\|_{2}^{2}+\left\|h \tilde{\tau}^{s^{\prime}} \nabla v_{\tilde{\tau}}\right\|_{2}^{2}\right) d \tilde{\tau} \\
& \leq C\left\|\left(w_{0}, v_{0}\right)\right\|_{H^{2}}^{2}+C \eta h^{2} \int_{\tilde{\tau}_{0}}^{t}\left\|\frac{\nabla w}{\tilde{\tau}^{s^{\prime}+1}}\right\|_{2}^{2} d \tilde{\tau}+C(\eta) \int_{\tilde{\tau}_{0}}^{t} M(\tilde{\tau}) d \tau,
\end{aligned}
$$

where $M(\tau)$ is defined in (3.2).

Step 2. The $H^{1}$-energy inequality of $(w, v)$.

By Lemma 2.5 with $k=1$ and Lemma 2.1-Lemma 2.3, we have that for small $\eta>0$,

$$
\begin{aligned}
& h\left\|\chi_{0} D w\right\|_{2}^{2}+h\left\|\tilde{\tau}^{s^{\prime}} \chi_{0} D v\right\|_{2}^{2}+\int_{\tilde{\tau}_{0}}^{t}\left(\left\|\tilde{\tau}^{s^{\prime}} \chi_{0} \nabla D v\right\|_{2}^{2}+\left\|\frac{\chi_{0} D w}{\tilde{\tau}^{s^{\prime}+m}}\right\|_{2}^{2}\right) d \tilde{\tau} \\
\leq & C \int_{\tilde{\tau}_{0}}^{t}\left(\left\|\tilde{\tau}^{s^{\prime}} \nabla v\right\|_{2}^{2}+\left\|h \tilde{\tau}^{s^{\prime}} v_{\tilde{\tau}}\right\|_{2}^{2}+\left\|\tilde{\tau}^{s^{\prime}} v\right\|_{2}^{2}+M(\tilde{\tau})\right) d \tilde{\tau}+C\left\|\left(w_{0}, v_{0}\right)\right\|_{H^{1}}^{2} \\
\leq & C\left\|\left(w_{0}, v_{0}\right)\right\|_{H^{1}}^{2}+C \eta h^{2} \int_{\tilde{\tau}_{0}}^{t}\left\|\frac{\nabla w}{\tilde{\tau}^{s^{\prime}+m}}\right\|_{2}^{2} d \tilde{\tau}+C(\eta) \int_{1}^{t} M(\tilde{\tau}) d \tilde{\tau} .
\end{aligned}
$$

From Lemma 2.6 with $k=1$, we see that

$$
\begin{aligned}
& h\left\|\chi_{1} \partial_{\Gamma} w\right\|_{2}^{2}+h\left\|\chi_{1} \tilde{\tau}^{s^{\prime}} \partial_{\Gamma} V\right\|_{2}^{2}+\int_{\tilde{\tau}_{0}}^{t}\left\|\tilde{\tau}^{s^{\prime}} \chi_{1} \bar{\nabla} \partial_{\Gamma} V\right\|_{2}^{2} d \tilde{\tau} \\
\leq & C\left\|\left(w_{0}, v_{0}\right)\right\|_{H^{1}}^{2}+C \eta \int_{1}^{t}\left\|\frac{\partial_{\Gamma} w}{\tilde{\tau}^{s^{\prime}+m}}\right\|_{2}^{2} d \tilde{\tau}+C(\eta) \int_{\tilde{\tau}_{0}}^{t}\left(\left\|\tilde{\tau}^{s^{\prime}} L\left(\partial_{\Gamma} V, 0\right)\right\|_{2}^{2}+M(\tilde{\tau})\right) d \tilde{\tau} .
\end{aligned}
$$


In addition, by Lemma 2.7 with $k=0$, we arrive at

$$
\begin{aligned}
& \quad h\left\|\chi_{1} \partial_{r} w\right\|_{2}^{2}+\int_{\tilde{\tau}_{0}}^{t}\left(\left\|\chi_{1} \tilde{\tau}^{s^{\prime}} \overline{\nabla d i v} V\right\|_{2}^{2}+\left\|\chi_{1} \frac{\partial_{r} w}{\tilde{\tau}^{s^{\prime}}+m}\right\|_{2}^{2}\right) d \tilde{\tau} \\
& \leq C\left\|\left(w_{0}, v_{0}\right)\right\|_{H^{1}}^{2}+C \eta \int_{\tilde{\tau}_{0}}^{t}\left\|\frac{\partial_{\Gamma} w}{\tilde{\tau}^{s^{\prime}+m}}\right\|_{2}^{2} d \tilde{\tau}+C(\eta) \int_{\tilde{\tau}_{0}}^{t}\left(\left\|\tilde{\tau}^{s^{\prime}} \chi_{1} L\left(\partial_{\Gamma} V, 1\right)\right\|_{2}^{2}\right. \\
& \left.\quad+\left\|h \tilde{\tau}^{s^{\prime}} \chi_{1} V_{\tilde{\tau}}\right\|_{2}^{2}+\left\|\tilde{\tau}^{s^{\prime}} \chi_{1} L(V, 1)\right\|_{2}^{2}+M(\tilde{\tau})\right) d \tilde{\tau} .
\end{aligned}
$$

Collecting (3.5)-(3.6) and (3.3) yields

$$
\begin{aligned}
& h\left\|\chi_{1} D w\right\|_{2}^{2}+\int_{\tilde{\tau}_{0}}^{t}\left\|\chi_{1} \tilde{\tau}^{s^{\prime}} \nabla d i v v\right\|_{2}^{2} d \tilde{\tau} \\
\leq & C\left\|\left(w_{0}, v_{0}\right)\right\|_{H^{1}}^{2}+C \eta \int_{\tilde{\tau}_{0}}^{t}\left\|\frac{\nabla w}{\tilde{\tau}^{s^{\prime}+m}}\right\|_{2}^{2}+C(\eta) \int_{\tilde{\tau}_{0}}^{t} M(\tilde{\tau}) d \tilde{\tau} .
\end{aligned}
$$

This, together with (3.4), yields

$$
\begin{gathered}
h\|D w\|_{2}^{2}+\int_{\tilde{\tau}_{0}}^{t}\left\|\tilde{\tau}^{s^{\prime}} \nabla d i v v\right\|_{2}^{2} d \tilde{\tau} \\
\leq C\left\|\left(w_{0}, v_{0}\right)\right\|_{H^{1}}^{2}+C \eta \int_{\tilde{\tau}_{0}}^{t}\left\|\frac{\nabla w}{\tilde{\tau}^{s^{\prime}}+m}\right\|_{2}^{2}+C(\eta) \int_{\tilde{\tau}_{0}}^{t} M(\tilde{\tau}) d \tilde{\tau} .
\end{gathered}
$$

Next, we rewrite (2.5a)-(2.5b) as

$$
\operatorname{div}\left(\tilde{\tau}^{s^{\prime}} v\right)=-\tilde{\tau}^{s^{\prime}+m} \frac{d w}{d \tilde{\tau}}+\tilde{\tau}^{s^{\prime}+m} f_{0}
$$

and

$$
-\mu \tilde{\tau}^{s^{\prime}} \Delta v+\frac{\gamma \nabla w}{\tilde{\tau}^{s^{\prime}+m}}=-(1+\alpha) h \tilde{\tau}^{s^{\prime}} v_{\tau}-\tilde{\tau}^{s^{\prime}-1} \alpha h v+(\mu+\lambda) \nabla \operatorname{div} v+\tilde{\tau}^{s^{\prime}} g .
$$

Then by Lemma 2.8 with $u=\mu \tilde{\tau}^{s^{\prime}} v$ and $P=\frac{\gamma w}{\tilde{\tau}^{s^{\prime}+m}}$, we obtain

$$
\begin{aligned}
& \left\|\tilde{\tau}^{s^{\prime}} D^{2} v\right\|_{2}^{2}+\left\|\frac{\nabla w}{\tilde{\tau}^{s^{\prime}}+m}\right\|_{2}^{2} \\
& \leq C\left\|\tilde{\tau}^{s^{\prime}+m} \frac{d w}{d \tilde{\tau}}\right\|_{H^{1}}^{2}+C\left\|\tilde{\tau}^{s^{\prime}+m} f_{0}\right\|_{H^{1}}^{2}+C\left\|h \tilde{\tau}^{s^{\prime}} v_{\tilde{\tau}}\right\|_{2}^{2} \\
& \quad+C\left\|\tilde{\tau}^{s-1} v\right\|_{2}^{2}+C\left\|\tilde{\tau}^{s^{\prime}} \nabla d i v v\right\|_{2}^{2}+C\left\|\tilde{\tau}^{s^{\prime}} g\right\|_{2}^{2} .
\end{aligned}
$$

From (2.5a), we see that

$$
D\left(\frac{d w}{d \tilde{\tau}}\right)=-\frac{1}{\tilde{\tau}^{m}} D d i v v+D f_{0} .
$$

Together with (3.7), this yields

$$
\int_{\tilde{\tau}_{0}}^{t}\left\|\tilde{\tau}^{s^{\prime}+m} D\left(\frac{d w}{d \tilde{\tau}}\right)\right\|_{2}^{2} d \tilde{\tau} \leq C\left\|\left(w_{0}, v_{0}\right)\right\|_{H^{1}}^{2}+C \eta \int_{\tilde{\tau}_{0}}^{t}\left\|\frac{\nabla w}{\tilde{\tau}^{s^{\prime}+m}}\right\|_{2}^{2}+C(\eta) \int_{\tilde{\tau}_{0}}^{t} M(\tilde{\tau}) d \tilde{\tau} .
$$


By applying Lemma 2.1 and (3.9), we arrive at

$$
\int_{\tilde{\tau}_{0}}^{t}\left\|\tilde{\tau}^{s^{\prime}+m} \frac{d w}{d \tilde{\tau}}\right\|_{H^{1}}^{2} d \tilde{\tau} \leq C\left\|\left(w_{0}, v_{0}\right)\right\|_{H^{1}}^{2}+C \eta \int_{\tilde{\tau}_{0}}^{t}\left\|\frac{\nabla w}{\tilde{\tau}^{s^{\prime}+m}}\right\|_{2}^{2}+C(\eta) \int_{\tilde{\tau}_{0}}^{t} M(\tilde{\tau}) d \tilde{\tau} .
$$

Substituting (3.3), (3.7), (3.10) into (3.8), we obtain

$$
\begin{aligned}
& \int_{\tilde{\tau}_{0}}^{t}\left(\left\|\tilde{\tau}^{s^{\prime}} D^{2} v\right\|_{2}^{2}+\left\|\frac{\nabla w}{\frac{\nabla w}{\tilde{\tau}^{\prime}+m}}\right\|_{2}^{2}\right) d \tilde{\tau} \\
\leq & C\left\|\left(w_{0}, v_{0}\right)\right\|_{H^{1}}^{2}+C \eta \int_{\tilde{\tau}_{0}}^{t}\left\|\frac{\nabla w}{\tilde{\tau}^{s^{\prime}+m}}\right\|_{2}^{2}+C(\eta) \int_{\tilde{\tau}_{0}}^{t} M(\tilde{\tau}) d \tilde{\tau} .
\end{aligned}
$$

This, together with (3.7), yields for small $\eta>0$

$$
h\|D w\|_{2}^{2}+\int_{\tilde{\tau}_{0}}^{t}\left(\left\|\tilde{\tau}^{s^{\prime}} D^{2} v\right\|_{2}^{2}+\left\|\frac{\nabla w}{\tilde{\tau}^{s^{\prime}}+m}\right\|_{2}^{2}\right) d \tilde{\tau} \leq C\left\|\left(w_{0}, v_{0}\right)\right\|_{H^{1}}^{2}+C \int_{\tilde{\tau}_{0}}^{t} M(\tilde{\tau}) d \tilde{\tau} .
$$

Step 3. The $H^{2}$-energy inequality of $(w, v)$.

At first, we have that from $(2.5 b)$

$$
-\mu \Delta v-(\mu+\lambda) \nabla d i v v=-(1+\alpha) h v_{\tilde{\tau}}-\frac{\alpha h}{\tilde{\tau}} v-\frac{\gamma}{\tilde{\tau}^{2 s^{\prime}+m}} \nabla w+g .
$$

By the regularity theory on the second order elliptic equation system, we obtain

$$
\begin{aligned}
\left\|\tilde{\tau}^{s^{\prime}} D^{2} v\right\|_{2}^{2} & \leq C \tilde{\tau}^{2 s^{\prime}}\left\|-h v_{\tilde{\tau}}-\frac{\alpha h}{\tilde{\tau}} v-\frac{\gamma}{\tilde{\tau}^{2 s^{\prime}+m}} \nabla w+g\right\|_{2}^{2} \\
& \leq C\left(\left\|h \tilde{\tau}^{s^{\prime}} v_{\tilde{\tau}}\right\|_{2}^{2}+\left\|\tilde{\tau}^{s^{\prime}} v\right\|_{2}^{2}+\|\nabla w\|_{2}^{2}+\left\|\tilde{\tau}^{s^{\prime}} g\right\|_{2}^{2}\right) .
\end{aligned}
$$

This, together with (3.3) and (3.11), yields

$$
\sup _{\tilde{\tau}_{0} \leq \tilde{\tau} \leq t} h\left\|\tilde{\tau}^{s^{\prime}} D^{2} v\right\|_{2}^{2} \leq C\left\|\left(w_{0}, v_{0}\right)\right\|_{H^{1}}^{2}+C \int_{\tilde{\tau}_{0}}^{t} M(\tilde{\tau}) d \tilde{\tau}+C \sup _{1 \leq \tau \leq t} h\left\|\tilde{\tau}^{s^{\prime}} g(\tilde{\tau})\right\|_{2}^{2} .
$$

By Lemma 2.5 with $k=2$, (3.11) and (3.3), we get for $t \geq \tilde{\tau}_{0}$

$$
h\left\|\chi_{0} D^{2} w\right\|_{2}^{2}+\int_{1}^{t}\left(\left\|\tilde{\tau}^{s^{\prime}} \chi_{0} D^{3} v\right\|_{2}^{2}+\left\|\frac{\chi_{0} D^{2} w}{\tilde{\tau}^{s^{\prime}}+m}\right\|_{2}^{2}\right) d \tilde{\tau} \leq C\left\|\left(w_{0}, v_{0}\right)\right\|_{H^{2}}^{2}+C \int_{1}^{t} M(\tilde{\tau}) d \tilde{\tau} .
$$

By Lemma 2.6 with $k=2$ and Lemma 2.7 with $k=1$, one has respectively

$$
\begin{aligned}
& h\left\|\chi_{1} \partial_{\Gamma}^{2} w\right\|_{2}^{2}+\int_{\tilde{\tau}_{0}}^{t}\left\|\tilde{\tau}^{s^{\prime}} \chi_{1} \bar{\nabla}\left(\partial_{\Gamma}^{2} V\right)\right\|_{2}^{2} d \tilde{\tau} \\
\leq & C\left\|\left(w_{0}, v_{0}\right)\right\|_{H^{2}}^{2}+C \eta \int_{\tilde{\tau}_{0}}^{t}\left\|\frac{\partial_{\Gamma}^{2} w}{\tilde{\tau}^{s^{\prime}}+m}\right\|_{2}^{2} d \tilde{\tau}+C(\eta) \int_{\tilde{\tau}_{0}}^{t} M(\tilde{\tau}) d \tilde{\tau}
\end{aligned}
$$


and

$$
\begin{aligned}
& h\left\|\chi_{1} \partial_{r} \partial_{\Gamma} w\right\|_{2}^{2}+\int_{\tilde{\tau}_{0}}^{t}\left(\left\|\tilde{\tau}^{s^{\prime}} \chi_{1} \bar{\nabla} \operatorname{div}\left(\partial_{\Gamma} V\right)\right\|_{2}^{2}+\left\|\chi_{1} \frac{\partial_{r} \partial_{\Gamma} w}{\tilde{\tau}^{s^{\prime}+m}}\right\|_{2}^{2}\right) d \tilde{\tau} \\
\leq & \left\|\left(w_{0}, v_{0}\right)\right\|_{H^{2}}^{2}+C \eta \int_{\tilde{\tau}_{0}}^{t}\left\|\frac{\partial_{\Gamma}^{2} w}{\tilde{\tau}^{s^{\prime}}+m}\right\|_{2}^{2} d \tilde{\tau}+C(\eta) \int_{\tilde{\tau}_{0}}^{t}\left(\left\|\tilde{\tau}^{s^{\prime}} \chi_{1} L\left(\partial_{\Gamma}^{2} V, 1\right)\right\|_{2}^{2}+M(\tilde{\tau})\right) d \tilde{\tau} .
\end{aligned}
$$

Combining (3.14) and (3.15), we see that

$$
\begin{aligned}
& h\left\|\chi_{1} \partial_{\Gamma} D w\right\|_{2}^{2}+\int_{\tilde{\tau}_{0}}^{t}\left\|\tilde{\tau}^{s^{\prime}} \chi_{1} \overline{\nabla \operatorname{div}}\left(\partial_{\Gamma} V\right)\right\|_{2}^{2} d \tilde{\tau} \\
\leq & C\left\|\left(w_{0}, v_{0}\right)\right\|_{H^{2}}^{2}+C \eta \int_{\tilde{\tau}_{0}}^{t}\left\|\frac{\partial_{\Gamma}^{2} w}{\tilde{\tau}^{s^{\prime}}+m}\right\|_{2}^{2} d \tilde{\tau}+C(\eta) \int_{\tilde{\tau}_{0}}^{t} M(\tilde{\tau}) d \tilde{\tau} .
\end{aligned}
$$

And combining (3.16) with (3.13), we obtain

$$
\begin{aligned}
& h\left\|\partial_{\Gamma} D w\right\|_{2}^{2}+\int_{\tilde{\tau}_{0}}^{t}\left\|\tilde{\tau}^{s^{\prime}} \nabla \operatorname{div}\left(\partial_{\Gamma} v\right)\right\|_{2}^{2} d \tilde{\tau} \\
\leq & C\left\|\left(w_{0}, v_{0}\right)\right\|_{H^{2}}^{2}+C \eta \int_{\tilde{\tau}_{0}}^{t}\left\|\frac{\partial_{\Gamma}^{2} w}{\tilde{\tau}^{s^{\prime}}+m}\right\|_{2}^{2} d \tilde{\tau}+C(\eta) \int_{\tilde{\tau}_{0}}^{t} M(\tilde{\tau}) d \tilde{\tau} .
\end{aligned}
$$

In addition, we can rewrite $\partial_{\Gamma}(2.5 \mathrm{a})$ and $\partial_{\Gamma}(2.5 \mathrm{~b})$ as

$$
\begin{aligned}
& \operatorname{div}\left(\tilde{\tau}^{s^{\prime}} \partial_{\Gamma} v\right)=-\tilde{\tau}^{s^{\prime}+m} \partial_{\Gamma}\left(\frac{d w}{d \tilde{\tau}}\right)+\tilde{\tau}^{s^{\prime}+m} \partial_{\Gamma} f_{0}+\left[\operatorname{div}, \partial_{\Gamma}\right] \tilde{\tau}^{s^{\prime}} v, \\
& \begin{array}{r}
-\tilde{\tau}^{s^{\prime}} \mu \Delta \partial_{\Gamma} v+\frac{\gamma \nabla \partial_{\Gamma} w}{\tilde{\tau}^{s^{\prime}+m}}=-\tilde{\tau}^{s^{\prime}} \mu\left[\Delta, \partial_{\Gamma}\right] v+\left[\nabla, \partial_{\Gamma}\right] \frac{\gamma w}{\tilde{\tau}^{s^{\prime}+m}}-\tilde{\tau}^{s^{\prime}}(1+\alpha) h \partial_{\Gamma} v_{\tau} \\
\quad-\tilde{\tau}^{s^{\prime}-1} \alpha h \partial_{\Gamma} v+\tilde{\tau}^{s^{\prime}}(\mu+\lambda) \partial_{\Gamma} \nabla d i v v+\tilde{\tau}^{s^{\prime}} \partial_{\Gamma} g .
\end{array}
\end{aligned}
$$

In order to apply Lemma 2.8 to estimate $\tilde{\tau}^{s^{\prime}} D^{2} \partial_{\Gamma} v$ and $\tilde{\tau}^{s^{\prime}} D \partial_{\Gamma} v$, we require to analyze each term in the right hand sides of (3.18a)-(3.18b). Ar first, from (2.5a) we see that

$$
\partial_{\Gamma} D\left(\frac{d w}{d \tilde{\tau}}\right)=-\frac{1}{\tilde{\tau}^{m}} \partial_{\Gamma} D d i v v+\partial_{\Gamma} D f_{0}
$$

and then by (3.17) we have

$$
\int_{\tilde{\tau}_{0}}^{t}\left\|\tilde{\tau}^{s^{\prime}+m} \partial_{\Gamma} D\left(\frac{d w}{d \tilde{\tau}}\right)\right\|_{2}^{2} d \tilde{\tau} \leq C\left\|\left(w_{0}, v_{0}\right)\right\|_{H^{2}}^{2}+C \eta \int_{\tilde{\tau}_{0}}^{t}\left\|\frac{\partial_{\Gamma}^{2} w}{\tilde{\tau}^{s^{\prime}+m}}\right\|_{2}^{2} d \tilde{\tau}+C(\eta) \int_{\tilde{\tau}_{0}}^{t} M(\tilde{\tau}) d \tilde{\tau} .
$$

This, together with (3.10)-(3.11), yields

$$
\int_{\tilde{\tau}_{0}}^{t}\left\|\tilde{\tau}^{s^{\prime}+m} \partial_{\Gamma}\left(\frac{d w}{d \tilde{\tau}}\right)\right\|_{H^{1}}^{2} d \tilde{\tau} \leq C\left\|\left(w_{0}, v_{0}\right)\right\|_{H^{2}}^{2}+C \eta \int_{\tilde{\tau}_{0}}^{t}\left\|\frac{\partial_{\Gamma}^{2} w}{\tilde{\tau}^{s^{\prime}+m}}\right\|_{2}^{2} d \tilde{\tau}+C(\eta) \int_{\tilde{\tau}_{0}}^{t} M(\tilde{\tau}) d \tilde{\tau}
$$


On the other hand, it is noted that

$$
\left\{\begin{array}{l}
\|\left[\text { div, } \partial_{\Gamma}\right] \tilde{\tau}^{s^{\prime}} v\left\|_{2}^{2} \leq C\right\| \tilde{\tau}^{s^{\prime}} \nabla v \|_{2}^{2} \\
\left\|\tilde{\tau}^{s^{\prime}}\left[\Delta, \partial_{\Gamma}\right] v\right\|_{2}^{2} \leq C\left\|\tilde{\tau}^{s^{\prime}} D^{2} v\right\|_{2}^{2} \\
\left\|\left[\nabla, \partial_{\Gamma}\right] \frac{w}{\tilde{\tau}^{s^{\prime}+1}}\right\|_{2}^{2} \leq C \| \frac{D w}{\frac{D w}{\tilde{\tau}^{s^{\prime}+1}} \|_{2}^{\prime}} \\
\left\|\tilde{\tau}^{s^{\prime}} \partial_{\Gamma} \nabla d i v v\right\|_{2}^{2} \leq C\left(\left\|\tilde{\tau}^{s^{\prime}} \nabla d i v \partial_{\Gamma} v\right\|_{2}^{2}+\left\|\tilde{\tau}^{s^{\prime}} D^{2} v\right\|_{2}^{2}\right) .
\end{array}\right.
$$

Then by applying Lemma 2.8 for (3.18a)-(3.18b) and using (3.19)-(3.20), we obtain

$$
\begin{aligned}
& \int_{\tilde{\tau}_{0}}^{t}\left(\left\|\tilde{\tau}^{s^{\prime}} D^{2} \partial_{\Gamma} v\right\|_{2}^{2}+\left\|\frac{\partial_{\Gamma} D w}{\tilde{\tau}^{s^{\prime}}+m}\right\|_{2}^{2}\right) d \tilde{\tau} \\
\leq & C\left\|\left(w_{0}, v_{0}\right)\right\|_{H^{2}}^{2}+C \eta \int_{\tilde{\tau}_{0}}^{t}\left\|\frac{\partial_{\Gamma}^{2} w}{\tilde{\tau}^{s^{\prime}+m}}\right\|_{2}^{2} d \tilde{\tau}+C(\eta) \int_{\tilde{\tau}_{0}}^{t} M(\tilde{\tau}) d \tilde{\tau},
\end{aligned}
$$

which means that for small $\eta>0$,

$$
\int_{\tilde{\tau}_{0}}^{t}\left(\left\|\tilde{\tau}^{s^{\prime}} D^{2} \partial_{\Gamma} v\right\|_{2}^{2}+\left\|\frac{\partial_{\Gamma} D w}{\tilde{\tau}^{s^{\prime}+m}}\right\|_{2}^{2}\right) d \tilde{\tau} \leq C\left\|\left(w_{0}, v_{0}\right)\right\|_{H^{2}}^{2}+C \int_{\tilde{\tau}_{0}}^{t} M(\tilde{\tau}) d \tilde{\tau} .
$$

Next, we estimate $\left\|D^{2} w\right\|_{2}^{2}$ and $\int_{\tilde{\tau}_{0}}^{t}\left\|\frac{D^{2} w}{\tilde{\tau}^{s^{\prime}+m}}\right\|_{2}^{2} d \tilde{\tau}$. It follows $\partial_{r}(2.63)$ with $k=0$ and direct computation that

$$
\begin{aligned}
& (1+\alpha) h\left(\partial_{r}^{2} w\right)_{\tilde{\tau}}+\frac{\gamma}{(2 \mu+\lambda) \tilde{\tau}^{2 s^{\prime}+2 m}} \partial_{r}^{2} w \\
= & \frac{1}{\tilde{\tau}^{m}} L\left(\partial_{\Gamma}^{1} V, 2\right)+\partial_{r}^{2} f+\frac{1}{\tilde{\tau}^{m}} L(V, 2)+\frac{1}{\tilde{\tau}^{m}}\left(-2 h \partial_{\tilde{\tau}}\left(\partial_{r} v_{r}\right)+\partial_{r} g_{r}\right. \\
& \left.\quad+L(V, 2)-\frac{\alpha h}{\tilde{\tau}} \partial_{r} v_{r}+L\left(\partial_{\Gamma}^{2} V, 1\right)\right) .
\end{aligned}
$$

Computing $\int_{S_{0}} \chi_{1}^{2}(3.22) \times \partial_{r}^{2} w d y$ yields for small $\eta_{1}>0$,

$$
\begin{aligned}
& \quad \frac{1+\alpha}{2} h \frac{d}{d \tilde{\tau}}\left\|\chi_{1} \partial_{r}^{2} w\right\|_{2}^{2}+\left\|\frac{\chi_{1} \partial_{r}^{2} w}{\tilde{\tau}^{s^{\prime}}+m}\right\|_{2}^{2} \\
& \leq C\left\|\tilde{\tau}^{s^{\prime}} L\left(\partial_{\Gamma} V, 2\right)\right\|_{2}^{2}+\left|\left(\chi_{1}^{2} \partial_{r}^{2} f, \partial_{r}^{2} w\right)\right|+C\left\|\tilde{\tau}^{s^{\prime}} L(V, 2)\right\|_{2}^{2} \\
& \quad+C\left\|h \tilde{\tau}^{s^{\prime}} \partial_{\tilde{\tau}}\left(\partial_{r} v_{r}\right)\right\|_{2}^{2}+C\left\|\tilde{\tau}^{s^{\prime}} \partial_{r} g_{r}\right\|_{2}^{2}+C\left\|\tilde{\tau}^{s^{\prime}-1} \partial_{r} v_{r}\right\|_{2}^{2}+\eta_{1}\left\|\frac{\chi_{1} \partial_{r}^{2} w}{\tilde{\tau}^{s^{\prime}+m}}\right\|_{2}^{2} .
\end{aligned}
$$

Integrating (3.23) with respect to the variable $\tilde{\tau}$ over $\left(\tilde{\tau}_{0}, t\right)$ and using the results in Lemma 2.6-Lemma 2.7, then we arrive at

$$
h\left\|\chi_{1} \partial_{r}^{2} w\right\|_{2}^{2}+\int_{\tilde{\tau}_{0}}^{t}\left\|\frac{\chi_{1} \partial_{r}^{2} w}{\tilde{\tau}^{s^{\prime}}+m}\right\|_{2}^{2} d \tilde{\tau} \leq C h\left\|\left(w_{0}, v_{0}\right)\right\|_{H^{2}}^{2}+C \int_{\tilde{\tau}_{0}}^{t} M(\tilde{\tau}) d \tilde{\tau} .
$$


Combining (3.13), (3.17), (3.21) and (3.24) yields

$$
h\left\|D^{2} w\right\|_{2}^{2}+\int_{\tilde{\tau}_{0}}^{t}\left(\left\|\tilde{\tau}^{s^{\prime}} D^{2} \partial_{\Gamma} v\right\|_{2}^{2}+\left\|\frac{D^{2} w}{\tilde{\tau}^{s^{\prime}}+m}\right\|_{2}^{2}\right) d \tilde{\tau} \leq C h\left\|\left(w_{0}, v_{0}\right)\right\|_{H^{2}}^{2}+C \int_{\tilde{\tau}_{0}}^{t} M(\tilde{\tau}) d \tilde{\tau} .
$$

Noting that $v$ satisfies the following second order elliptic equation system

$$
\left\{\begin{array}{l}
-\mu \Delta v-(\mu+\lambda) \nabla d i v v=-(1+\alpha) h v_{\tilde{\tau}}-\frac{\alpha h}{\tilde{\tau}} v-\frac{\gamma}{\tilde{\tau}^{2 s^{\prime}+m}} \nabla w+g, \\
v=0 \text { on } S_{0},
\end{array}\right.
$$

we then have

$$
\|v\|_{H^{3}} \leq C\left\|-(1+\alpha) h v_{\tilde{\tau}}-\frac{\alpha h}{\tilde{\tau}} v-\frac{\gamma}{\tilde{\tau}^{2 s^{\prime}+m}} \nabla w+g\right\|_{H^{1}}
$$

which implies

$$
\left\|\tilde{\tau}^{s^{\prime}} v\right\|_{H^{3}}^{2} \leq C\left(\left\|h \tilde{\tau}^{s^{\prime}} v_{\tilde{\tau}}\right\|_{H^{1}}^{2}+\left\|\tilde{\tau}^{s^{\prime}-1} v\right\|_{H^{1}}^{2}+\left\|\tilde{\tau}^{s^{\prime}} g\right\|_{H^{1}}^{2}+\left\|\frac{\nabla w}{\tilde{\tau}^{s^{\prime}+m}}\right\|_{H^{1}}^{2}\right) .
$$

Combining (3.12), (3.25) and (3.28), we arrive at

$$
\begin{aligned}
& h\left\|\tilde{\tau}^{s^{\prime}} D^{2} v\right\|_{2}^{2}+h\left\|D^{2} w\right\|_{2}^{2}+\int_{\tilde{\tau}_{0}}^{t}\left(\left\|\tilde{\tau}^{s^{\prime}} D^{3} v\right\|_{2}^{2}+\left\|\frac{D^{2} w}{\tilde{\tau}^{s^{\prime}+m}}\right\|_{2}^{2}\right) d \tilde{\tau} \\
\leq & C\left\|\left(w_{0}, v_{0}\right)\right\|_{H^{2}}^{2}+C \int_{\tilde{\tau}_{0}}^{t} M(\tilde{\tau}) d \tilde{\tau}+C \sup _{\tilde{\tau}_{0} \leq \tilde{\tau} \leq t} h\left\|\tilde{\tau}_{0}^{s^{\prime}} g(\tilde{\tau})\right\|_{2}^{2} .
\end{aligned}
$$

Thus, by (3.3), (3.11) and (3.29), we have obtained

$$
N_{2}\left(\tilde{\tau}_{0}, t\right) \leq C\left\|\left(w_{0}, v_{0}\right)\right\|_{H^{2}}^{2}+C \int_{\tilde{\tau}_{0}}^{t} M(\tilde{\tau}) d \tilde{\tau}+C \sup _{\tilde{\tau}_{0} \leq \tilde{\tau} \leq t} h\left\|\tilde{\tau}^{s^{\prime}} g(\tilde{\tau})\right\|_{2}^{2} .
$$

Step 4. The $H^{3}$-energy inequality of $(w, v)$.

By Lemma 2.4 and (3.30), we have

$$
\begin{aligned}
& h\left\|h \tilde{\tau}^{s^{\prime}} \nabla v_{\tilde{\tau}}\right\|_{2}^{2}+\int_{\tilde{\tau}_{0}}^{t}\left(\left\|h^{2} w_{\tilde{\tau} \tilde{\tau}}\right\|_{2}^{2}+\left\|h^{2} \tilde{\tau}^{s^{\prime}} v_{\tilde{\tau} \tilde{\tau}}\right\|_{2}^{2}\right) d \tilde{\tau} \\
\leq & C\left\|\left(w_{0}, v_{0}\right)\right\|_{H^{3}}^{2}+C \int_{\tilde{\tau}_{0}}^{t} M(\tilde{\tau}) d \tilde{\tau}+C \sup _{\tilde{\tau}_{0} \leq \tilde{\tau} \leq t} h\left\|\tilde{\tau}^{s^{\prime}} g(\tilde{\tau})\right\|_{2}^{2} .
\end{aligned}
$$

Due to (3.28) and (3.30), one has

$$
h\left\|\tilde{\tau}^{s^{\prime}} v\right\|_{H^{3}}^{2} \leq C\left\|\left(w_{0}, v_{0}\right)\right\|_{H^{3}}^{2}+C \int_{\tilde{\tau}_{0}}^{t} M(\tilde{\tau}) d \tilde{\tau}+C \sup _{\tilde{\tau}_{0} \leq \tilde{\tau} \leq t} h\left\|\tilde{\tau}^{s^{\prime}} g(\tilde{\tau})\right\|_{H^{1}}^{2} .
$$

On the other hand, it follows from (3.26) that

$$
-\mu \Delta v_{\tilde{\tau}}-(\mu+\lambda) \nabla d i v v_{\tilde{\tau}}=-(1+\alpha) h v_{\tilde{\tau} \tilde{\tau}}-\left(\frac{\alpha h}{\tilde{\tau}} v\right)_{\tilde{\tau}}-\left(\frac{\gamma}{\tilde{\tau} 2 s^{\prime}+m} \nabla w\right)_{\tilde{\tau}}+g_{\tilde{\tau}} .
$$


Noting that we can obtain for

$$
\begin{aligned}
& \left\|-(1+\alpha) h v_{\tilde{\tau} \tilde{\tau}}-\left(\frac{\alpha h}{\tilde{\tau}} v\right)_{\tilde{\tau}}-\left(\frac{\gamma}{\tilde{\tau}^{2 s^{\prime}+m}} \nabla w\right)_{\tilde{\tau}}+g_{\tilde{\tau}}\right\|_{2} \\
\leq C & \left(\left\|h v_{\tilde{\tau} \tilde{\tau}}\right\|_{2}+\left\|\tilde{\tau}^{-1} v_{\tilde{\tau}}\right\|_{2}+\left\|\tilde{\tau}^{-2} v\right\|_{2}+\left\|\frac{\nabla w_{\tilde{\tau}}}{\tilde{\tau}^{2 s^{\prime}+m}}\right\|_{2}+\left\|\frac{\nabla w}{\tilde{\tau}^{2 s^{\prime}+m+1}}\right\|_{2}+\left\|g_{\tilde{\tau}}\right\|_{2}\right) .
\end{aligned}
$$

Then it follows from (3.33)-(3.34) and a direct computation that

$$
\begin{aligned}
& \left\|h \tilde{\tau}^{s^{\prime}} D^{2} v_{\tilde{\tau}}\right\|_{2}^{2} \\
& \leq C\left(\left\|h^{2} \tilde{\tau}^{s^{\prime}} v_{\tilde{\tau} \tilde{\tau}}\right\|_{2}^{2}+\left\|h \tilde{\tau}^{s^{\prime}-1} v_{\tilde{\tau}}\right\|_{2}^{2}+\left\|h \tilde{\tau}^{s^{\prime}-2} v\right\|_{2}^{2}\right. \\
& \left.\quad+\left\|h \tilde{\tau}^{s^{\prime}} g_{\tilde{\tau}}\right\|_{2}^{2}+\left\|h \tilde{\tau}^{s^{\prime}} D^{2} v\right\|_{2}^{2}+\left\|h \frac{\nabla w}{\tilde{\tau}^{s^{\prime}+m}}\right\|_{2}+\left\|h \frac{\nabla w_{\tilde{\tau}}}{\tilde{\tau}^{s^{\prime}+m}}\right\|_{2}\right) .
\end{aligned}
$$

This, together with (3.30)-(3.31), yields

$$
\int_{\tilde{\tau}_{0}}^{t}\left\|h \tilde{\tau}^{s^{\prime}} D^{2} v_{\tilde{\tau}}\right\|_{2}^{2} d \tilde{\tau} \leq C\left\|\left(w_{0}, v_{0}\right)\right\|_{H^{3}}^{2}+C \int_{\tilde{\tau}_{0}}^{t} M(\tilde{\tau}) d \tilde{\tau}+C \sup _{\tilde{\tau}_{0} \leq \tilde{\tau} \leq t} h\left\|\tilde{\tau}^{s^{\prime}} g(\tilde{\tau})\right\|_{2}^{2} .
$$

We now focus on the estimates on $D^{3} w$. At first, by Lemma 2.5 with $k=3$, we have such an interior estimate

$$
\begin{aligned}
& h\left\|\chi_{0} D^{3} w\right\|_{2}^{2}+\int_{\tilde{\tau}_{0}}^{t}\left(\left\|\tilde{\tau}^{s^{\prime}} \chi_{0} D^{4} v\right\|_{2}^{2}+\left\|\frac{\chi_{0} D^{3} w}{\tilde{\tau}^{s^{\prime}+m}}\right\|_{2}^{2}\right) d \tilde{\tau} \\
\leq & C\left\|\left(w_{0}, v_{0}\right)\right\|_{H^{3}}^{2}+C \int_{\tilde{\tau}_{0}}^{t} M(\tilde{\tau}) d \tilde{\tau}+C \sup _{\tilde{\tau}_{0} \leq \tilde{\tau} \leq t} h\left\|\tilde{\tau}^{s^{\prime}} g(\tilde{\tau})\right\|_{2}^{2} .
\end{aligned}
$$

In addition, by Lemma 2.6 with $k=3$ and Lemma 2.7 with $k=2$, we have

$$
\begin{aligned}
& h\left\|\chi_{1} \partial_{\Gamma}^{2} D w\right\|_{2}^{2}+\int_{\tilde{\tau}_{0}}^{t}\left(\left\|\tilde{\tau}^{s^{\prime}} \chi_{1} \nabla\left(\partial_{\Gamma}^{3} v\right)\right\|_{2}^{2}+\left\|\tilde{\tau}^{s^{\prime}} \chi_{1} \nabla \operatorname{div}\left(\partial_{\Gamma}^{2} v\right)\right\|_{2}^{2}+\left\|\frac{\chi_{1} \partial_{r} \partial_{\Gamma}^{2} w}{\tilde{\tau}^{s^{\prime}}+m}\right\|_{2}^{2}\right) d \tilde{\tau} \\
\leq & C\left\|\left(w_{0}, v_{0}\right)\right\|_{H^{3}}^{2}+C \eta \int_{\tilde{\tau}_{0}}^{t}\left\|\frac{D^{3} w}{\tilde{\tau}^{s^{\prime}+m}}\right\|_{2}^{2} d \tilde{\tau}+C(\eta) \int_{\tilde{\tau}_{0}}^{t}\left(\left\|\tilde{\tau}^{s^{\prime}} D v\right\|_{H^{2}}^{2}+\left\|h \tilde{\tau}^{s^{\prime}} D^{2} v_{\tilde{\tau}}\right\|_{2}^{2}+M(\tilde{\tau})\right) d \tilde{\tau} \\
\leq & C\left\|\left(w_{0}, v_{0}\right)\right\|_{H^{3}}^{2}+C \eta \int_{\tilde{\tau}_{0}}^{t}\left\|\frac{D^{3} w}{\tilde{\tau}^{s^{\prime}+m}}\right\|_{2}^{2} d \tilde{\tau}+C(\eta) \int_{\tilde{\tau}_{0}}^{t} M(\tilde{\tau}) d \tilde{\tau}+C(\eta) \sup _{\tilde{\tau}_{0} \leq \tilde{\tau} \leq t} h\left\|\tilde{\tau}^{s^{\prime}} g(\tilde{\tau})\right\|_{2}^{2} .
\end{aligned}
$$

Combining (3.37) and (3.38) yields

$$
\begin{aligned}
& h\left\|\partial_{\Gamma}^{2} D w\right\|_{2}^{2}+\int_{\tilde{\tau}_{0}}^{t}\left(\left\|\tilde{\tau}^{s^{\prime}} \nabla\left(\partial_{\Gamma}^{3} v\right)\right\|_{2}^{2}+\left\|\tilde{\tau}^{s^{\prime}} \nabla \operatorname{div}\left(\partial_{\Gamma}^{2} v\right)\right\|_{2}^{2}+\left\|\frac{\partial_{r} \partial_{\Gamma}^{2} w}{\tilde{\tau}^{s^{\prime}}+m}\right\|_{2}^{2}\right) d \tilde{\tau} \\
\leq & C\left\|\left(w_{0}, v_{0}\right)\right\|_{H^{3}}^{2}+C \eta \int_{\tilde{\tau}_{0}}^{t}\left\|\frac{D^{3} w}{\tilde{\tau}^{s^{\prime}}+m}\right\|_{2}^{2} d \tilde{\tau}+C(\eta) \int_{\tilde{\tau}_{0}}^{t} M(\tilde{\tau}) d \tilde{\tau}+C(\eta) \sup _{\tilde{\tau}_{0} \leq \tilde{\tau} \leq t} h\left\|\tilde{\tau}^{s^{\prime}} g(\tilde{\tau})\right\|_{2}^{2} .
\end{aligned}
$$


In addition, we rewrite $\partial_{\Gamma}^{2}(2.5 \mathrm{a})$ and $\partial_{\Gamma}^{2}(2.5 \mathrm{~b})$ as

$$
\left\{\begin{array}{r}
\operatorname{div}\left(\tilde{\tau}^{s^{\prime}} \partial_{\Gamma}^{2} v\right)=-\tilde{\tau}^{s^{\prime}+m} \partial_{\Gamma}^{2}\left(\frac{d w}{d \tilde{\tau}}\right)+\tilde{\tau}^{s^{\prime}+m} \partial_{\Gamma}^{2} f_{0}+\left[\operatorname{div}, \partial_{\Gamma}^{2}\right] \tilde{\tau}^{s^{\prime}} v \\
-\tilde{\tau}^{s^{\prime}} \mu \Delta \partial_{\Gamma}^{2} v+\frac{\gamma \nabla \partial_{\Gamma}^{2} w}{\tilde{\tau}^{s^{\prime}+m}}=-\tilde{\tau}^{s^{\prime}} \mu\left[\Delta, \partial_{\Gamma}^{2}\right] v+\left[\nabla, \partial_{\Gamma}^{2}\right] \frac{\gamma w}{\tilde{\tau}^{s^{\prime}+m}}-(1+\alpha) h \tilde{\tau}^{s^{\prime}} \partial_{\Gamma}^{2} v_{\tilde{\tau}} \\
\quad-\tilde{\tau}^{s^{\prime}-1} \alpha h \partial_{\Gamma}^{2} v+(\mu+\lambda) \tilde{\tau}^{s^{\prime}} \partial_{\Gamma}^{2} \nabla d i v v+\tilde{\tau}^{s^{\prime}} \partial_{\Gamma}^{2} g
\end{array}\right.
$$

As in (iii), in order to apply Lemma 2.8 to estimate $\tilde{\tau}^{s^{\prime}} D^{2} \partial_{\Gamma}^{2} v$ and $\tilde{\tau}^{s^{\prime}} D\left(\frac{\partial_{\Gamma}^{2} w}{\tilde{\tau}^{\prime}+1}\right)$, we require to analyze the terms in the right hand sides of (3.40). At first, from (2.5a) we see that

$$
\partial_{\Gamma}^{2} D\left(\frac{d w}{d \tilde{\tau}}\right)=-\frac{1}{\tilde{\tau}^{m}} \partial_{\Gamma}^{2} D d i v v+\partial_{\Gamma}^{2} D f_{0}
$$

Together with (3.39), this yields

$$
\begin{aligned}
& \int_{\tilde{\tau}_{0}}^{t}\left\|\tilde{\tau}^{s^{\prime}+m} \partial_{\Gamma}^{2} D\left(\frac{d w}{d \tilde{\tau}}\right)\right\|_{2}^{2} d \tilde{\tau} \\
\leq C & \left(w_{0}, v_{0}\right)\left\|_{H^{3}}^{2}+C \eta \int_{\tilde{\tau}_{0}}^{t}\right\| \frac{D^{3} w}{\tilde{\tau}^{s^{\prime}+m}}\left\|_{2}^{2} d \tilde{\tau}+C(\eta) \int_{\tilde{\tau}_{0}}^{t} M(\tilde{\tau}) d \tilde{\tau}+C(\eta) \sup _{\tilde{\tau}_{0} \leq \tilde{\tau} \leq t} h\right\| \tilde{\tau}^{s^{\prime}} g(\tilde{\tau}) \|_{2}^{2} .
\end{aligned}
$$

Collecting (3.19) and (3.41), we have

$$
\begin{aligned}
& \int_{\tilde{\tau}_{0}}^{t}\left\|\tilde{\tau}^{s^{\prime}+m} \partial_{\Gamma}^{2}\left(\frac{d w}{d \tilde{\tau}}\right)\right\|_{H^{1}}^{2} d \tilde{\tau} \\
\leq C & \left\|\left(w_{0}, v_{0}\right)\right\|_{H^{3}}^{2}+C \eta \int_{\tilde{\tau}_{0}}^{t}\left\|\frac{D^{3} w}{\tilde{\tau}^{s^{\prime}+m}}\right\|_{2}^{2} d \tilde{\tau}+C(\eta) \int_{\tilde{\tau}_{0}}^{t} M(\tilde{\tau}) d \tilde{\tau}+C(\eta) \sup _{\tilde{\tau}_{0} \leq \tilde{\tau} \leq t} h\left\|\tilde{\tau}^{s^{\prime}} g(\tilde{\tau})\right\|_{2}^{2} .
\end{aligned}
$$

It follows from direct computation that

$$
\left\{\begin{array}{l}
\|\left[\text { div, } \partial_{\Gamma}^{2}\right] \tilde{\tau}^{s^{\prime}} v\left\|_{2}^{2} \leq C\right\| \tilde{\tau}^{s^{\prime}} D^{2} v \|_{2}^{2} \\
\left\|\tilde{\tau}^{s^{\prime}}\left[\Delta, \partial_{\Gamma}^{2}\right] v\right\|_{2}^{2} \leq C\left\|\tilde{\tau}^{s^{\prime}} D^{3} v\right\|_{2}^{2} \\
\left\|\left[\nabla, \partial_{\Gamma}^{2}\right] \frac{w}{\tilde{\tau}^{s^{\prime}+1}}\right\|_{2}^{2} \leq C\left\|\frac{D^{2} w}{\tilde{\tau}^{s^{\prime}+1}}\right\|_{2}^{2} \\
\| \tilde{\tau}^{s^{\prime}} \partial_{\Gamma}^{2} \nabla \text { divv } \|_{2}^{2} \leq C\left(\left\|\tilde{\tau}^{s^{\prime}} \nabla d i v \partial_{\Gamma}^{2} v\right\|_{2}^{2}+\left\|\tilde{\tau}^{s^{\prime}} D^{3} v\right\|_{2}^{2}\right) .
\end{array}\right.
$$

By Lemma 2.8 for (3.40) and the results in Lemma 2.6-Lemma 2.10, we arrive at

$$
\begin{aligned}
& \int_{\tilde{\tau}_{0}}^{t}\left(\left\|\tilde{\tau}^{s^{\prime}} D^{2} \partial_{\Gamma}^{2} v\right\|_{2}^{2}+\left\|\frac{\partial_{\Gamma}^{2} D w}{\tilde{\tau}^{s^{\prime}}+m}\right\|_{2}^{2}\right) d \tilde{\tau} \\
\leq C & \left\|\left(w_{0}, v_{0}\right)\right\|_{H^{3}}^{2}+C \eta \int_{\tilde{\tau}_{0}}^{t}\left\|\frac{D^{3} w}{\tilde{\tau}^{s^{\prime}}+m}\right\|_{2}^{2} d \tilde{\tau}+C(\eta) \int_{\tilde{\tau}_{0}}^{t} M(\tilde{\tau}) d \tilde{\tau}+C(\eta) \sup _{\tilde{\tau}_{0} \leq \tilde{\tau} \leq t} h\left\|\tilde{\tau}^{s^{\prime}} g(\tilde{\tau})\right\|_{2}^{2} .
\end{aligned}
$$


On the other hand, we rewrite $\partial_{r}(2.48 \mathrm{a}),(2.48 \mathrm{~b})$ and $(2.48 \mathrm{c})$ as

$$
\begin{aligned}
& (1+\alpha) h\left(\partial_{r} w\right)_{\tilde{\tau}}+\frac{1}{\tilde{\tau}^{m}} \partial_{r}^{2} v_{r}=\partial_{r} f+\frac{1}{\tilde{\tau}^{m}} L(V, 1)+\frac{1}{\tilde{\tau}^{m}}\left(\frac{1}{r} \partial_{r \theta}^{2} v_{\theta}+\frac{1}{r \sin \theta} \partial_{r \varphi}^{2} v_{\varphi}\right), \\
& -(2 \mu+\lambda) \partial_{r}^{2} v_{r}+\frac{\gamma}{\tilde{\tau}^{2 s^{\prime}+m}} \partial_{r} w \\
& =-(1+\alpha) h \partial_{\tilde{\tau}} v_{r}+L\left(\partial_{\Gamma}^{2} v_{r}, 0\right)+L\left(\partial_{r} \partial_{\Gamma} V_{\Gamma}, 0\right)+g_{r}+L(V, 1), \\
& -\partial_{r}^{2} V_{\Gamma}=-(1+\alpha) h \partial_{\tilde{\tau}} V_{\Gamma}+L\left(\partial_{\Gamma}^{2} V_{\Gamma}, 0\right)+L\left(\partial_{r} \partial_{\Gamma} V, 0\right)-\frac{\gamma \bar{\nabla}^{\prime} w}{\tilde{\tau}^{2 s^{\prime}+m}}+g_{\Gamma}+L(V, 1) .
\end{aligned}
$$

By $\partial_{r}^{2}\left[(3.45 a)+\frac{1}{\tilde{\tau}^{m}(2 \mu+\lambda)}(3.45 b)\right]$ and direct computation, we have

$$
\begin{aligned}
& (1+\alpha) h\left(\partial_{r}^{3} w\right)_{\tilde{\tau}}+\frac{\gamma}{2 \mu+\lambda} \frac{\gamma}{\tilde{\tau}^{2 s^{\prime}+2 m}} \partial_{r}^{3} w \\
= & \partial_{r}^{3} f+\frac{1}{\tilde{\tau}^{m}} L(V, 3)+\frac{1}{\tilde{\tau}^{m}} L\left(\partial_{r}^{3} \partial_{\Gamma} V_{\Gamma}, 0\right) \\
& \quad+\frac{1}{\tilde{\tau}^{m}(2 \mu+\lambda)}\left(-(1+\alpha) h \partial_{\tilde{\tau}} \partial_{r}^{2} v_{r}+L\left(\partial_{\Gamma}^{2} \partial_{r}^{2} v_{r}, 0\right)+L\left(\partial_{r}^{3} \partial_{\Gamma} V_{\Gamma}, 0\right)+\partial_{r}^{2} g_{r}+L(V, 3)\right) .
\end{aligned}
$$

Computing $\int_{S_{0}} \chi_{1}^{2}(3.46) \times \partial_{r}^{3} w d y$ yields that for small $\eta_{1}>0$,

$$
\begin{aligned}
& \quad h \frac{d}{d \tilde{\tau}}\left\|\chi_{1} \partial_{r}^{3} w\right\|_{2}^{2}+\left\|\frac{\chi_{1} \partial_{r}^{3} w}{\tilde{\tau}^{s^{\prime}+m}}\right\|_{2}^{2} \\
& \leq C\left\|\tilde{\tau}^{s^{\prime}} D v\right\|_{H^{2}}^{2}+\left|\left(\chi_{1}^{2} \partial_{r}^{3} f, \partial_{r}^{3} w\right)\right| \\
& \quad+C\left\|\tilde{\tau}^{s^{\prime}} L\left(\partial_{r}^{3} \partial_{\Gamma} V_{\Gamma}, 0\right)\right\|_{2}^{2}+C\left\|\tilde{\tau}^{s^{\prime}} h \partial_{\tau} D^{2} v\right\|_{2}^{2}+C M(\tilde{\tau})+\eta_{1}\left\|\frac{\chi_{1} \partial_{r}^{3} w}{\tilde{\tau}^{s^{\prime}+m}}\right\|_{2}^{2} .
\end{aligned}
$$

Integrating (3.47) over $\left(\tilde{\tau}_{0}, t\right)$ and applying the results in Lemma 2.6-Lemma 2.10, we have

$$
\begin{aligned}
& h\left\|\chi_{1} \partial_{r}^{3} w\right\|_{2}^{2}+\int_{\tilde{\tau}_{0}}^{t}\left\|\frac{\chi_{1} \partial_{r}^{3} w}{\tilde{\tau}^{s^{\prime}}+m}\right\|_{2}^{2} d \tilde{\tau} \\
\leq & C\left\|\left(w_{0}, v_{0}\right)\right\|_{H^{3}}^{2}+C \int_{\tilde{\tau}_{0}}^{t}\left(\left\|\tilde{\tau}^{s^{\prime}} L\left(\partial_{r}^{3} \partial_{\Gamma} V_{\Gamma}, 0\right)\right\|_{2}^{2}+M(\tilde{\tau})\right) d \tilde{\tau}+C \sup _{\tilde{\tau}_{0} \leq \tilde{\tau} \leq t} h\left\|\tilde{\tau}^{s^{\prime}} g(\tilde{\tau})\right\|_{2}^{2} .
\end{aligned}
$$

From the equation $\partial_{r} \partial_{\Gamma}(3.45 \mathrm{c})$, we see that

$$
\begin{aligned}
-\partial_{r}^{3} \partial_{\Gamma} V_{\Gamma}= & -(1+\alpha) h \partial_{\tau} \partial_{r} \partial_{\Gamma} V_{\Gamma}+L\left(\partial_{\Gamma}^{3} \partial_{r} V_{\Gamma}, 0\right)+L\left(\partial_{r}^{2} \partial_{\Gamma}^{2} V, 0\right) \\
& -\frac{\gamma \partial_{r} \partial_{\Gamma} \bar{\nabla}^{\prime} w}{\tilde{\tau}^{2 s^{\prime}+m}}+\partial_{r} \partial_{\Gamma} g_{\Gamma}+L(V, 3),
\end{aligned}
$$

which means

$$
\begin{aligned}
& \left\|\tilde{\tau}^{s^{\prime}} \partial_{r}^{3} \partial_{\Gamma} V_{\Gamma}\right\|_{2}^{2} \\
\leq C & \left.C h \tilde{\tau}^{s^{\prime}} D^{2} v_{\tilde{\tau}}\left\|_{2}^{2}+\right\| \tilde{\tau}^{s^{\prime}} \partial_{\Gamma}^{2} D^{2} v\left\|_{2}^{2}+\right\| \tilde{\tau}^{s^{\prime}} D^{2} g v\left\|_{2}^{2}+\right\| \tilde{\tau}^{s^{\prime}} D^{3} v\left\|_{2}^{2}+\right\| \frac{\partial_{\Gamma}^{2} D w}{\tilde{\tau}^{s^{\prime}+m}} \|_{2}^{2}\right) .
\end{aligned}
$$


Substituting (3.49) into (3.48) and using (3.28), we have

$$
\begin{aligned}
& h\left\|\chi_{1} \partial_{r}^{3} w\right\|_{2}^{2}+\int_{\tilde{\tau}_{0}}^{t}\left\|\frac{\chi_{1} \partial_{r}^{3} w}{\tilde{\tau}^{s^{\prime}}+m}\right\|_{2}^{2} d \tilde{\tau} \\
\leq & C\left\|\left(w_{0}, v_{0}\right)\right\|_{H^{3}}^{2}+C(\eta) \int_{\tilde{\tau}_{0}}^{t} M(\tilde{\tau}) d \tilde{\tau}+C \eta \int_{\tilde{\tau}_{0}}^{t}\left\|\frac{D^{3} w}{\tilde{\tau}^{s^{\prime}+m}}\right\|_{2}^{2} d \tilde{\tau}+C(\eta) \sup _{\tilde{\tau}_{0} \leq \tilde{\tau} \leq t} h\left\|\tilde{\tau}^{s^{\prime}} g(\tilde{\tau})\right\|_{2}^{2} .
\end{aligned}
$$

Combining (3.39), (3.44) with (3.48)-(3.50), we arrive at

$$
\begin{aligned}
& h\left\|D^{3} w\right\|_{2}^{2}+\int_{\tilde{\tau}_{0}}^{t}\left(\left\|\tilde{\tau}^{s^{\prime}} D^{4} v\right\|_{2}^{2}+\left\|\frac{D^{3} w}{\tilde{\tau}^{s^{\prime}+m}}\right\|_{2}^{2}\right) d \tilde{\tau} \\
\leq & C\left\|\left(w_{0}, v_{0}\right)\right\|_{H^{3}}^{2}+C \int_{\tilde{\tau}_{0}}^{t} M(\tilde{\tau}) d \tilde{\tau}+C \sup _{\tilde{\tau}_{0} \leq \tilde{\tau} \leq t} h\left\|\tilde{\tau}^{s^{\prime}} g(\tilde{\tau})\right\|_{2}^{2},
\end{aligned}
$$

which derives together with (3.30)

$$
N_{3}\left(\tilde{\tau}_{0}, t\right) \leq C\left\|\left(w_{0}, v_{0}\right)\right\|_{H^{3}}^{2}+C \int_{\tilde{\tau}_{0}}^{t} M(\tilde{\tau}) d \tilde{\tau}+C \sup _{\tilde{\tau}_{0} \leq \tilde{\tau} \leq t} h\left\|\tilde{\tau}^{s^{\prime}} g(\tilde{\tau})\right\|_{H^{1}}^{2} .
$$

Based on the above results in Step 1-Step 4, we now establish the following conclusion:

Proposition 3.1. Assuming $\gamma<\frac{2}{3}+\frac{1}{\alpha}$ and $N_{3}\left(\tilde{\tau}_{0}, t\right)<1$, then for $t \geq \tilde{\tau}_{0}$, we have

$$
N_{3}\left(\tilde{\tau}_{0}, t\right) \leq C h\left\|\left(w_{0}, v_{0}\right)\right\|_{H^{3}}^{2}+C h^{2}+C\left(h^{-\frac{1}{2}} N_{3}^{\frac{3}{2}}\left(\tilde{\tau}_{0}, t\right)+h^{-1} N_{3}^{2}\left(\tilde{\tau}_{0}, t\right)\right) .
$$

Proof. To prove (3.52), we require to estimate all the terms in right hand side of (3.51). By the expression of $M(\tilde{\tau})$, we only need to treat the terms such as $\left|\left(D^{3} f, D^{3} w\right)\right|,\left\|\tilde{\tau}^{s^{\prime}+m} f_{0}\right\|_{H^{3}}^{2}$, $\left\|\tilde{\tau}^{s^{\prime}} g\right\|_{H^{2}}^{2},\left\|h \tilde{\tau}^{s^{\prime}} g_{\tilde{\tau}}\right\|_{2}^{2}$ and $\left\|h f_{\tilde{\tau}}\right\|_{2}^{2}$.

For $\left(D^{3} f, D^{3} w\right)$, we see that

$$
\begin{aligned}
\left(D^{3} f, D^{3} w\right) & =-\int_{S_{0}} \frac{1}{\tilde{\tau}^{m}}\left(D^{3}(w d i v v) D^{3} w+D^{3}(v \cdot \nabla w) D^{3} w\right) d y \\
& =-\int_{S_{0}} \frac{1}{\tilde{\tau}^{m}}\left(D^{3}(w d i v v) D^{3} w+\left[D^{3}, v \cdot \nabla\right] w D^{3} w+v \cdot \nabla D^{3} w D^{3} w\right) d y \\
& =-\int_{S_{0}} \frac{1}{\tilde{\tau}^{m}}\left(D^{3}(w d i v v) D^{3} w+\left[D^{3}, v \cdot \nabla\right] w D^{3} w-\frac{1}{2} \operatorname{div} v\left(D^{3} w\right)^{2}\right) d y
\end{aligned}
$$

Next we analyze each term in the right hand side of (3.53). Note that

$$
\begin{aligned}
& \int_{S_{0}} \frac{1}{\tilde{\tau}^{m}}\left(D^{3}(w d i v v) D^{3} w\right) d y \\
= & \frac{1}{\tilde{\tau}^{m}} \int_{S_{0}}\left(D^{3} w \operatorname{div} v D^{3} w+3 D^{2} w D \operatorname{div} v D^{3} w+3 D w D^{2} \operatorname{div} v D^{3} w+w D^{3} \operatorname{div} v D^{3} w\right) d y .
\end{aligned}
$$


In addition, since

$$
\begin{aligned}
\frac{1}{\tilde{\tau}^{m}}\left|\int_{S_{0}}\left(D^{3} w d i v v D^{3} w\right) d y\right| & \leq C \| \tilde{\tau}^{s^{\prime}} \text { divv }\left\|_{L^{\infty}}\right\| \frac{D^{3} w}{\tilde{\tau}^{s^{\prime}+m}}\left\|_{2}\right\| D^{3} w \|_{2} \\
& \leq C\left\|\tilde{\tau}^{s^{\prime}} d i v v\right\|_{H^{2}}\left\|\frac{D^{3} w}{\tilde{\tau}^{s^{\prime}+m}}\right\|_{2}\left\|D^{3} w\right\|_{2} \\
& \leq C\left(\left\|\tilde{\tau}^{s^{\prime}} d i v v\right\|_{H^{2}}^{2}+\left\|\frac{D^{3} w}{\tilde{\tau}^{s^{\prime}+m}}\right\|_{2}^{2}\right)\left\|D^{3} w\right\|_{2}
\end{aligned}
$$

we have

$$
\begin{aligned}
& \int_{\tilde{\tau}_{0}}^{t}\left|\frac{1}{\tilde{\tau}^{m}} \int_{S_{0}}\left(D^{3} w d i v v D^{3} w\right) d y\right| d \tilde{\tau} \\
\leq & C \int_{\tilde{\tau}_{0}}^{t}\left(\left\|\tilde{\tau}^{s^{\prime}} d i v v\right\|_{H^{2}}^{2}+\left\|\frac{D^{3} w}{\tilde{\tau}^{s^{\prime}+m}}\right\|_{2}^{2}\right) \sup _{\tilde{\tau}_{0} \leq \tilde{\tau} \leq t}\left\|D^{3} w(\tilde{\tau})\right\|_{2} \\
\leq & C h^{-\frac{1}{2}} N_{3}^{\frac{3}{2}}\left(\tilde{\tau}_{0}, t\right) .
\end{aligned}
$$

Similarly,

$$
\begin{aligned}
& \frac{1}{\tilde{\tau}^{m}}\left|\int_{S_{0}}\left(D^{2} w D \operatorname{div} v D^{3} w\right) d y\right| \\
\leq & C\left\|\tilde{\tau}^{s^{\prime}} D d i v v\right\|_{L^{\infty}}\left\|\frac{D^{2} w}{\tilde{\tau}^{s^{\prime}+m}}\right\|_{2}\left\|D^{3} w\right\|_{2} \\
\leq & C\left(\left\|\tilde{\tau}^{s^{\prime}} \operatorname{div}\right\|_{H^{3}}^{2}+\left\|\frac{D^{2} w}{\tilde{\tau}^{s^{\prime}+m}}\right\|_{2}^{2}\right)\left\|D^{3} w\right\|_{2},
\end{aligned}
$$

which concludes

$$
\int_{\tilde{\tau}_{0}}^{t}\left|\frac{1}{\tilde{\tau}^{m}} \int_{S_{0}}\left(D^{2} w D d i v v D^{3} w\right) d y\right| d \tilde{\tau} \leq C h^{-\frac{1}{2}} N_{3}^{\frac{3}{2}}(1, t) .
$$

We also have

$$
\begin{aligned}
& \int_{\tilde{\tau}_{0}}^{t}\left|\frac{1}{\tilde{\tau}^{m}} \int_{S_{0}}\left(D w D^{2} d i v v D^{3} w\right) d y\right| d \tilde{\tau} \\
\leq & C \int_{1}^{t}\left\|\tilde{\tau}^{s^{\prime}} D^{2} d i v v\right\|_{L^{2}}\left\|\frac{D^{3} w}{\tilde{\tau}^{s+m}}\right\|_{2}\|D w\|_{L^{\infty}} d \tilde{\tau} \\
\leq & C \int_{\tilde{\tau}_{0}}^{t}\left(\left\|\tilde{\tau}^{s^{\prime}} d i v v\right\|_{H^{3}}^{2}+\left\|\frac{D^{3} w}{\tilde{\tau}^{s^{\prime}+m}}\right\|_{2}^{2}\right) d \tilde{\tau} \sup _{\tilde{\tau}_{0} \leq \tilde{\tau} \leq t}\|D w\|_{H^{2}} \\
\leq & C h^{-\frac{1}{2}} N_{3}^{\frac{3}{2}}\left(\tilde{\tau}_{0}, t\right)
\end{aligned}
$$


and

$$
\begin{aligned}
& \int_{\tilde{\tau}_{0}}^{t}\left|\frac{1}{\tilde{\tau}^{m}} \int_{S_{0}}\left(w D^{3} d i v v D^{3} w\right) d y\right| d \tilde{\tau} \\
\leq & C \int_{\tilde{\tau}_{0}}^{t}\left\|\tilde{\tau}^{s^{\prime}} D^{3} d i v v\right\|_{L^{2}}\left\|\frac{D^{3} w}{\tilde{\tau}^{s^{\prime}+m}}\right\|_{2}\|w\|_{L^{\infty}} d \tilde{\tau} \\
\leq & C \int_{\tilde{\tau}_{0}}^{t}\left(\left\|\tilde{\tau}^{s^{\prime}} d i v v\right\|_{H^{3}}^{2}+\left\|\frac{D^{3} w}{\tilde{\tau}^{s^{\prime}+m}}\right\|_{2}^{2}\right) d \tilde{\tau} \sup _{\tilde{\tau}_{0} \leq \tilde{\tau} \leq t}\|w\|_{H^{2}} \leq C h^{-\frac{1}{2}} N_{3}^{\frac{3}{2}}(1, t) .
\end{aligned}
$$

Substituting (3.55)-(3.58) into (3.54) yields

$$
\int_{\tilde{\tau}_{0}}^{t}\left|\int_{S_{0}} \frac{1}{\tilde{\tau}^{m}}\left(D^{3}(w d i v v) D^{3} w\right) d y\right| d \tilde{\tau} \leq C h^{-\frac{1}{2}} N_{3}^{\frac{3}{2}}\left(\tilde{\tau}_{0}, t\right) .
$$

Analogously, we can arrive at

$$
\int_{\tilde{\tau}_{0}}^{t}\left|\frac{1}{\tilde{\tau}^{m}} \int_{S_{0}}\left(\left[D^{3}, v \cdot \nabla\right] w D^{3} w-\frac{1}{2} \operatorname{divv}\left(D^{3} w\right)^{2}\right) d y\right| d \tilde{\tau} \leq C h^{-\frac{1}{2}} N_{3}^{\frac{3}{2}}\left(\tilde{\tau}_{0}, t\right) .
$$

Inserting (3.59)-(3.60) into (3.53), we have

$$
\int_{\tilde{\tau}_{0}}^{t} \frac{1}{\tilde{\tau}^{m}}\left|\left(D^{3} f, D^{3} w\right)\right| d \tilde{\tau} \leq C h^{-\frac{1}{2}} N_{3}^{\frac{3}{2}}\left(\tilde{\tau}_{0}, t\right) .
$$

For the term $\left\|\tilde{\tau}^{s^{\prime}+m} f_{0}\right\|_{H^{3}}^{2}$, it suffices to treat $\left\|\tilde{\tau}^{s^{\prime}+m} D^{3} f_{0}\right\|_{2}^{2}$ since the other left terms in $\left\|\tilde{\tau}^{s^{\prime}+m} f_{0}\right\|_{H^{3}}^{2}$ can be more easily estimated. It follows from direct computation that

$$
\begin{aligned}
& \quad\left\|\tilde{\tau}^{s^{\prime}+m} D^{3} f_{0}\right\|_{2}^{2} \\
& =\tilde{\tau}^{2 s^{\prime}} \int_{S_{0}}\left|D^{3} w d i v v\right|^{2} d y+3 \tilde{\tau}^{2 s^{\prime}} \int_{S_{0}}\left|D^{2} w D d i v v\right|^{2} d y \\
& \quad+3 \tilde{\tau}^{2 s^{\prime}} \int_{S_{0}}\left|D w D^{2} d i v v\right|^{2} d y+\tilde{\tau}^{2 s^{\prime}} \int_{S_{0}}\left|w D^{3} d i v v\right|^{2} d y \\
& \leq C\left(\left\|\tilde{\tau}^{s^{\prime}} d i v v\right\|_{L^{\infty}}^{2}+\left\|\tilde{\tau}^{s^{\prime}} D d i v v\right\|_{L^{\infty}}^{2}\right)\left\|D^{2} w\right\|_{H^{1}}^{2} \\
& \quad+C\left\|\tilde{\tau}^{s^{\prime}} D^{2} d i v v\right\|_{H^{1}}^{2}\left(\|w\|_{L^{\infty}}^{2}+\|D w\|_{L^{\infty}}^{2}\right) \\
& \leq C\left\|\tilde{\tau}^{s^{\prime}} D v\right\|_{H^{3}}^{2}\|w\|_{H^{3}}^{2}
\end{aligned}
$$

which derives

$$
\int_{\tilde{\tau}_{0}}^{t}\left\|\tilde{\tau}^{s+m} D^{3} f_{0}\right\|_{2}^{2} d \tilde{\tau} \leq C h^{-1} N_{3}^{2}\left(\tilde{\tau}_{0}, t\right)
$$

For the treatment on the term $\left\|\tilde{\tau}^{s^{\prime}} g\right\|_{H^{2}}^{2}$ it is only enough to estimate $\left\|\tilde{\tau}^{s^{\prime}} D^{2} g\right\|_{2}^{2}$. Note that

$$
g=-\frac{1}{\tilde{\tau}^{m}} v \cdot \nabla v-\frac{1}{\tilde{\tau}^{m+2 s^{\prime}}} \nabla P_{1}(w)+\alpha(1-\alpha) h^{2} \tilde{\tau}^{-\frac{2}{1+\alpha}} y-\frac{w}{1+w} L v
$$


To estimate $\left\|\tilde{\tau}^{s^{\prime}} D^{2} g\right\|_{2}^{2}$, we need to deal with each term in the expression of $\tilde{\tau}^{s^{\prime}} D^{2} g$. It follows from Hölder inequality, Sobolev's imbedding inequality, and direct computation that

$$
\int_{\tilde{\tau}_{0}}^{t}\left\|\tilde{\tau}^{s^{\prime}} D^{2}\left(\frac{1}{\tilde{\tau}^{m}} v \cdot \nabla v\right)\right\|_{2}^{2} d \tilde{\tau} \leq C h^{-1} N_{3}^{2}\left(\tilde{\tau}_{0}, t\right)
$$

In addition,

$$
\begin{aligned}
& \left\|\tilde{\tau}^{s^{\prime}} D^{2}\left(\frac{1}{\tilde{\tau}^{2 s^{\prime}+m}} \nabla P_{1}(w)\right)\right\|_{2}^{2} \\
\leq C & \left\|\frac{D w}{\tilde{\tau}^{s^{\prime}+m}}\right\|_{H^{2}}^{2}\left(\|w\|_{L^{\infty}}^{2}+\|D w\|_{L^{\infty}}^{2}\right) \\
\leq C & \frac{D w}{\tilde{\tau}^{s^{\prime}+m}}\left\|_{H^{2}}^{2}\right\| w \|_{H^{3}}^{2}
\end{aligned}
$$

which derives

$$
\left.\int_{\tilde{\tau}_{0}}^{t}\left\|\tilde{\tau}^{s^{\prime}} D^{2}\left(\frac{1}{\tilde{\tau}^{2 s^{\prime}+m}} \nabla P_{1}(w)\right)\right\|_{2}^{2} d \tilde{\tau} \leq C h^{-1} N_{3}^{2}\left(\tilde{\tau}_{0}, t\right)\right) .
$$

For the term $\left\|\tilde{\tau}^{s^{\prime}} \frac{w}{1+w} L v\right\|_{H^{2}}^{2}$, we have

$$
\begin{aligned}
& \left\|\tilde{\tau}^{s^{\prime}} D^{2}\left(\frac{w}{1+w} L v\right)\right\|_{2}^{2} \\
\leq & C\left(\|w\|_{L^{\infty}}^{2}+\|D w\|_{L^{\infty}}^{2}\right)\left\|\tilde{\tau}^{s^{\prime}} D v\right\|_{H^{2}}^{2}+C\left\|\tilde{\tau}^{s^{\prime}} D^{2} w D^{2} v\right\|_{2}^{2} \\
\leq & C\|w\|_{H^{3}}^{2}\left\|\tilde{\tau}^{s^{\prime}} D v\right\|_{H^{2}}^{2}+C\left\|D^{2} w\right\|_{2}^{2}\left\|\tilde{\tau}^{s^{\prime}} D^{2} v\right\|_{L^{\infty}}^{2} \\
\leq & C\|w\|_{H^{3}}^{2}\left\|\tilde{\tau}^{s^{\prime}} D v\right\|_{H^{3}}^{2}
\end{aligned}
$$

which derives

$$
\int_{\tilde{\tau}_{0}}^{t}\left\|\tilde{\tau}^{s^{\prime}} D^{2}\left(\frac{w}{1+w} L v\right)\right\|_{2}^{2} d \tilde{\tau} \leq C \sup _{\tilde{\tau}_{0} \leq \tilde{\tau} \leq t}\|w\|_{H^{3}}^{2} \int_{0}^{t}\left\|\tilde{\tau}^{s^{\prime}} D v\right\|_{H^{3}}^{2} d \tilde{\tau} \leq C h^{-1} N_{3}^{2}\left(\tilde{\tau}_{0}, t\right) .
$$

For the term $\alpha(1-\alpha) h^{2} \tilde{\tau}^{-\frac{2}{1+\alpha}} y$, we see that

$$
\int_{\tilde{\tau}_{0}}^{t}\left\|\tilde{\tau}^{s^{\prime}} \alpha(1-\alpha) h^{2} \tilde{\tau}^{-\frac{2}{1+\alpha}} y\right\|_{H^{2}}^{2} d \tilde{\tau} \leq C h^{2} \int_{\tilde{\tau}_{0}}^{t} \tilde{\tau}^{2 s^{\prime}-\frac{4}{1+\alpha}} d \tilde{\tau} .
$$

Note that the integrand function $\tilde{\tau}^{2 s^{\prime}-\frac{4}{1+\alpha}} \in L^{1}(0,+\infty)$ is required, one then has

$$
2 s^{\prime}-\frac{4}{1+\alpha}+1=\frac{3 \alpha(\gamma-1)-3+\alpha}{1+\alpha}<0,
$$

which means

$$
\gamma<\frac{2}{3}+\frac{1}{\alpha}
$$


In this case,

$$
\int_{\tilde{\tau}_{0}}^{t}\left\|\tilde{\tau}^{s^{\prime}} \alpha(1-\alpha) h^{2} \tilde{\tau}^{-\frac{2}{1+\alpha}} y\right\|_{H^{2}}^{2} d \tilde{\tau} \leq C h^{2} .
$$

Thus, by (3.64)-(3.67) and (3.63), we arrive at

$$
\int_{\tilde{\tau}_{0}}^{t}\left\|\tilde{\tau}^{s^{\prime}} g\right\|_{H^{2}}^{2} d \tilde{\tau} \leq C h^{2}+C\left(h^{-\frac{1}{2}} N_{3}^{\frac{3}{2}}\left(\tilde{\tau}_{0}, t\right)+h^{-1} N_{3}^{2}\left(\tilde{\tau}_{0}, t\right)\right) .
$$

Next, we treat $\left\|h \tilde{\tau}^{s^{\prime}} g_{\tilde{\tau}}\right\|_{2}^{2}$. By the expression of $g$ in (3.63), we require to deal with the terms $\tilde{\tau}^{s^{\prime}}\left(\frac{1}{\tilde{\tau}^{m}} v \cdot \nabla v\right) \tilde{\tau}$, and $\tilde{\tau}^{s^{\prime}}\left(\frac{\nabla P_{1}(w)}{\tilde{\tau}^{2 s^{\prime}+m}}\right) \tilde{\tau}, \tilde{\tau}^{s^{\prime}}\left(\frac{w}{1+w} L v\right) \tilde{\tau}$, separately. It follows from direct computation that

$$
\begin{aligned}
\left\|\tilde{\tau}^{s^{\prime}}\left(\frac{1}{\tilde{\tau}^{m}} v \cdot \nabla v\right)_{\tilde{\tau}}\right\|_{2}^{2} & \leq C\left(\left\|\tilde{\tau}^{s^{\prime}-m-1} v \cdot \nabla v\right\|_{2}^{2}+\left\|\tilde{\tau}^{s^{\prime}-m} v_{\tilde{\tau}} \cdot \nabla v\right\|_{2}^{2}+\left\|\tilde{\tau}^{s^{\prime}-m} v \cdot \nabla v v_{\tilde{\tau}}\right\|_{2}^{2}\right. \\
& \leq\left(\|v\|_{L^{\infty}}^{2}+\|D v\|_{L^{\infty}}^{2}\right)\left(\left\|\tilde{\tau}^{s^{\prime}} v\right\|_{2}^{2}+\left\|\tilde{\tau}^{s^{\prime}} v_{\tilde{\tau}}\right\|_{2}^{2}+\left\|\tilde{\tau}^{s^{\prime}} D v_{\tilde{\tau}}\right\|_{2}^{2}\right) \\
& \leq C\left\|\tilde{\tau}^{s^{\prime}} v\right\|_{H^{3}}^{2}\left(\left\|\tilde{\tau}^{s^{\prime}} v\right\|_{2}^{2}+\left\|\tilde{\tau}^{s^{\prime}} v_{\tilde{\tau}}\right\|_{2}^{2}+\left\|\tilde{\tau}^{s^{\prime}} D v_{\tilde{\tau}}\right\|_{2}^{2}\right)
\end{aligned}
$$

which means

$$
\int_{\tilde{\tau}_{0}}^{t}\left\|h \tilde{\tau}^{s^{\prime}}\left(\frac{1}{\tilde{\tau}^{m}} v \cdot \nabla v\right)_{\tilde{\tau}}\right\|_{2}^{2} d \tilde{\tau} \leq C h^{-1} N_{3}^{2}\left(\tilde{\tau}_{0}, t\right)
$$

And

$$
\begin{aligned}
\left\|\tilde{\tau}^{s^{\prime}}\left(\frac{\nabla P_{1}(w)}{\tilde{\tau}^{2 s^{\prime}+m}}\right)_{\tilde{\tau}}\right\|_{2}^{2} & \leq C\left\|\frac{1}{\tilde{\tau}^{s^{\prime}+m+1}} \nabla P_{1}(w)\right\|_{2}^{2}+C\left\|\frac{1}{\tilde{\tau}^{s^{\prime}+m}}\left(\nabla P_{1}(w)\right)_{\tilde{\tau}}\right\|_{2}^{2} \\
& \leq C\left(\|w\|_{L^{\infty}}^{2}\left\|\frac{D w}{\tilde{\tau}^{s^{\prime}+m}}\right\|_{2}^{2}+\left\|\frac{D w}{\tilde{\tau}^{s^{\prime}+m}} w_{\tilde{\tau}}\right\|_{2}^{2}+\left\|\frac{D w_{\tilde{\tau}}}{\tilde{\tau}^{s^{\prime}+m}} w\right\|_{2}^{2}\right) \\
& \leq C\left(\|w\|_{L^{\infty}}^{2}\left\|\frac{D w}{\tilde{\tau}^{s^{\prime}+m}}\right\|_{2}^{2}+\|D w\|_{L^{\infty}}^{2}\left\|w_{\tilde{\tau}}\right\|_{2}^{2}+\left\|D w_{\tilde{\tau}}\right\|_{2}^{2}\|w\|_{L^{\infty}}^{2}\right),
\end{aligned}
$$

which derives

$$
\int_{\tilde{\tau}_{0}}^{t}\left\|h \tilde{\tau}^{s^{\prime}}\left(\frac{\nabla P_{1}(w)}{\tilde{\tau}^{2 s^{\prime}+m}}\right)_{\tilde{\tau}}\right\|_{2}^{2} d \tau \leq C h^{-1} N_{3}^{2}\left(\tilde{\tau}_{0}, t\right) .
$$

In addition, we have

$$
\begin{aligned}
& \left\|\tilde{\tau}^{s^{\prime}}\left(\frac{w}{1+w} L v\right)_{\tilde{\tau}}\right\|_{2}^{2} \\
& \leq C\left(\left\|w_{\tilde{\tau}}\right\|_{\infty}^{2}+\|w\|_{\infty}^{2}\right)\left(\left\|\tilde{\tau}^{s^{\prime}} D^{2} v\right\|_{2}^{2}+\left\|\tilde{\tau}^{s^{\prime}} D^{2} v_{\tilde{\tau}}\right\|_{2}^{2}\right),
\end{aligned}
$$

which implies

$$
\begin{aligned}
& \int_{\tilde{\tau}_{0}}^{t}\left\|h \tilde{\tau}^{s}\left(\frac{w}{1+w} L v\right)_{\tilde{\tau}}\right\|_{2}^{2} d \tilde{\tau} \\
\leq & C\left(\|w\|_{H^{3}}^{2}+\left\|h w_{\tilde{\tau}}\right\|_{H^{2}}^{2}\right) \int_{\tilde{\tau}_{0}}^{t}\left(\left\|\tilde{\tau}^{s^{\prime}} D^{2} v\right\|_{2}^{2}+\left\|h \tilde{\tau}^{s^{\prime}} D^{2} v_{\tilde{\tau}}\right\|_{2}^{2}\right) d \tilde{\tau} \\
\leq & C h^{-1} N_{3}^{2}\left(\tilde{\tau}_{0}, t\right) .
\end{aligned}
$$


Collecting (3.69)-(3.72) yields

$$
\int_{\tilde{\tau}_{0}}^{t}\left\|h \tilde{\tau}^{s^{\prime}} g_{\tilde{\tau}}\right\|_{2}^{2} d \tilde{\tau} \leq C h^{2}+C h^{-1} N_{3}^{2}\left(\tilde{\tau}_{0}, t\right) .
$$

Next, we estimate the term $\left\|h f_{\tilde{\tau}}\right\|_{2}^{2}$. It follows from the expression of $f$ and direct computation that

$$
\begin{aligned}
& \left\|\frac{1}{\tilde{\tau}^{m}}(\operatorname{div}(v w))_{\tilde{\tau}}\right\|_{2}^{2} \\
\leq & \left\|\frac{1}{\tilde{\tau}^{m}} w_{\tilde{\tau}} \operatorname{div} v\right\|_{2}^{2}+\left\|\frac{1}{\tilde{\tau}^{m}} w d i v v_{\tilde{\tau}}\right\|_{2}^{2}+\left\|\frac{1}{\tilde{\tau}^{m}} \nabla w w_{\tilde{\tau}} \cdot v\right\|_{2}^{2}+\left\|\frac{1}{\tilde{\tau}^{m}} \nabla w \cdot v_{\tilde{\tau}}\right\|_{2}^{2} \\
\leq & C\left(\|\operatorname{div} v\|_{L^{\infty}}^{2}+\|v\|_{l^{\infty}}^{2}+\|w\|_{L^{\infty}}+\|\nabla w\|_{L^{\infty}}\right)\left(\left\|w_{\tilde{\tau}}\right\|_{2}^{2}+\left\|\operatorname{div} v_{\tilde{\tau}}\right\|_{2}^{2}+\left\|\nabla w_{\tilde{\tau}}\right\|_{2}^{2}+\left\|v_{\tilde{\tau}}\right\|_{2}^{2}\right) \\
\leq & C\left(\|v\|_{H^{3}}^{2}+\|w\|_{H^{3}}^{2}\right)\left(\left\|w_{\tilde{\tau}}\right\|_{H^{1}}^{2}+\left\|v_{\tilde{\tau}}\right\|_{H^{1}}^{2},\right.
\end{aligned}
$$

which derives

$$
\int_{\tilde{\tau}_{0}}^{t}\left\|h f_{\tilde{\tau}}\right\|_{2}^{2} d \tilde{\tau} \leq C h^{-1} N_{3}^{2}\left(\tilde{\tau}_{0}, t\right) .
$$

Finally, we estimate the term $\sup _{\tilde{\tau}_{0} \leq \tilde{\tau} \leq t} h\left\|\tilde{\tau}^{s^{\prime}} g\right\|_{H^{1}}^{2}$. As in the above, we require to treat each term in the expression of $\tilde{\tau}^{s^{\prime}} D g$. It follows from direct computation that

$$
\begin{aligned}
& \sup _{\tau_{0} \leq \tilde{\tau} \leq t} h\left\|\tilde{\tau}^{s^{\prime}-m} D(v \cdot \nabla v)\right\|_{2}^{2} \\
\leq & C h\left(\left\|\tilde{\tau}^{s^{\prime}} D v \cdot \nabla v\right\|_{2}^{2}+\left\|\tilde{\tau}^{s^{\prime}} v \cdot \nabla D v\right\|_{2}^{2}\right) \\
\leq & C h \sup _{\tilde{\tau}_{0} \leq \tilde{\tau} \leq t}\left(\left\|\tilde{\tau}^{s^{\prime}} D v\right\|_{L^{\infty}}^{2}\left\|\tilde{\tau}^{s^{\prime}} D v\right\|_{2}^{2}+\left\|\tilde{\tau}^{s^{\prime}} D v\right\|_{L^{\infty}}^{2}\left\|\tilde{\tau}^{s^{\prime}} D^{2} v\right\|_{2}^{2}\right) \\
\leq & C h \sup _{\tilde{\tau}_{0} \leq \tilde{\tau} \leq t}\left(\left\|\tilde{\tau}^{s^{\prime}} D v\right\|_{H^{2}}^{2}\left\|\tilde{\tau}^{s^{\prime}} D v\right\|_{H^{1}}^{2}\right) \\
\leq & C h^{-1} N_{3}^{2}\left(\tilde{\tau}_{0}, t\right)
\end{aligned}
$$

and

$$
\begin{aligned}
& h\left\|\tilde{\tau}^{s^{\prime}} \frac{\gamma}{\tilde{\tau}^{2 s^{\prime}+m}} D\left(\nabla P_{1}(w)\right)\right\|_{2}^{2} \leq C h\|w\|_{H^{3}}^{4} \leq C h^{-1} N_{3}^{2}\left(\tilde{\tau}_{0}, t\right), \\
& h\left\|\tilde{\tau}^{s^{\prime}} D\left(\frac{w}{1+w} L v\right)\right\|_{2}^{2} \leq C\left\|\tilde{\tau}^{s^{\prime}} D\left(\frac{w}{1+w} D^{2} v\right)\right\|_{2}^{2} \\
& \left.\leq C h\left(\left\|\tilde{\tau}^{s^{\prime}} D w D^{2} v\right\|_{2}^{2}+\| \tilde{\tau}^{s^{\prime}} w D^{3} v\right) \|_{2}^{2}\right) \leq C h\|w\|_{H^{3}}^{2}\left\|\tilde{\tau}^{s^{\prime}} v\right\|_{H^{3}}^{2} \\
& \leq C h^{-1} N_{3}^{2}\left(\tilde{\tau}_{0}, t\right) .
\end{aligned}
$$

Collecting (3.75)-(3.76b) together with the expression of $g$ in (3.63), we get

$$
\sup _{\tilde{\tau}_{0} \leq \tilde{\tau} \leq t} h\left\|\tilde{\tau}^{s^{\prime}} g\right\|_{H^{1}}^{2} \leq C h^{2}+C h^{-1} N_{3}^{2}\left(\tilde{\tau}_{0}, t\right) .
$$

Combining (3.61)-(3.62), (3.68), (3.73)-(3.74) and (3.77), we complete the proof of (3.52), i.e., Proposition 3.1 is proved. 


\section{The proof of Theorem 1.2}

In this section, we will complete the proof of Theorem 1.2 based on the results in Section 2-Section 3. Denote by $t^{\prime}=\frac{1}{h}\left(t^{\frac{1}{1+\alpha}}-1\right)$ and define $\bar{N}_{k}\left(1, t^{\prime}\right)=h^{-1} N_{k}\left(\tilde{\tau}_{0}, t\right)$. Then we see that

$$
\begin{aligned}
& h \bar{N}_{k}\left(1, t^{\prime}\right)=N_{k}\left(\tilde{\tau}_{0}, t\right) \\
=\sup _{1 \leq \tau \leq t^{\prime}}\left(h\|w\|_{H^{k}}^{2}+h\left\|R^{s} v\right\|_{H^{k}}^{2}+h\left\|\frac{1}{(1+\alpha) R} w_{\tau}\right\|_{H^{k-1}}^{2}+h\left\|\frac{1}{(1+\alpha) R} R^{s} v_{\tau}\right\|_{H^{k-2}}^{2}\right) & \quad \int_{1}^{t^{\prime}}\left(\left\|\frac{D w}{R^{s+2}}\right\|_{H^{k-1}}^{2}+\left\|\frac{1}{(1+\alpha) R} w_{\tau}\right\|_{H^{k-1}}^{2}+\left\|R^{s} D v\right\|_{H^{k}}^{2}+\left\|\frac{1}{(1+\alpha) R} R^{s} D v_{\tau}\right\|_{H^{k-2}}^{2}\right. \\
& \left.+\left\|\frac{1}{(1+\alpha) R} R^{s} v_{\tau}\right\|_{2}^{2}\right)(1+\alpha) R h d \tau \\
= & \sup _{1 \leq \tau \leq t^{\prime}}\left(\|w\|_{H^{k}}^{2}+\left\|R^{s} v\right\|_{H^{k}}^{2}+\left\|\frac{1}{(1+\alpha) R} w_{\tau}\right\|_{H^{k-1}}^{2}+\left\|\frac{1}{(1+\alpha)} R^{s-1} v_{\tau}\right\|_{H^{k-2}}^{2}\right) \\
& +(1+\alpha) h \int_{1}^{t^{\prime}}\left(\left\|\frac{D w}{R^{s+\frac{3}{2}}}\right\|_{H^{k-1}}^{2}+\left\|\frac{1}{1+\alpha} R^{-\frac{1}{2}} w_{\tau}\right\|_{H^{k-1}}^{2}+\left\|R^{s+\frac{1}{2}} D v\right\|_{H^{k}}^{2}\right. \\
& \left.+\left\|\frac{1}{1+\alpha} R^{s-\frac{1}{2}} D v_{\tau}\right\|_{H^{k-2}}^{2}+\left\|\frac{1}{1+\alpha} R^{s-\frac{1}{2}} v_{\tau}\right\|_{2}^{2}\right) d \tau .
\end{aligned}
$$

Thus

$$
\begin{aligned}
& \bar{N}_{k}\left(1, t^{\prime}\right) \\
& =\sup _{1 \leq \tau \leq t^{\prime}}\left(\|w\|_{H^{k}}^{2}+\left\|R^{s} v\right\|_{H^{k}}^{2}+\left\|\frac{1}{(1+\alpha) R} w_{\tau}\right\|_{H^{k-1}}^{2}+\left\|\frac{1}{(1+\alpha)} R^{s-1} v_{\tau}\right\|_{H^{k-2}}^{2}\right) \\
& \quad+(1+\alpha) \int_{1}^{t^{\prime}}\left(\left\|\frac{D w}{R^{s+\frac{3}{2}}}\right\|_{H^{k-1}}^{2}+\left\|\frac{1}{1+\alpha} R^{-\frac{1}{2}} w_{\tau}\right\|_{H^{k-1}}^{2}+\left\|R^{s+\frac{1}{2}} D v\right\|_{H^{k}}^{2}\right. \\
& \left.\quad+\left\|\frac{1}{1+\alpha} R^{s-\frac{1}{2}} D v_{\tau}\right\|_{H^{k-2}}^{2}+\left\|\frac{1}{1+\alpha} R^{s-\frac{1}{2}} v_{\tau}\right\|_{2}^{2}\right) d \tau .
\end{aligned}
$$

By the same proof of Proposition 3.1, we can obtain

Proposition 4.1. If $\bar{N}_{3}\left(1, t^{\prime}\right) \leq 1, \gamma<\frac{2}{3}+\frac{1}{\alpha}$ and $t^{\prime} \geq 1$, then we have

$$
\bar{N}_{3}\left(1, t^{\prime}\right) \leq C\left\|\left(w_{0}, v_{0}\right)\right\|_{H^{3}}^{2}+C h+C \bar{N}_{3}^{\frac{3}{2}}\left(1, t^{\prime}\right) .
$$

Based on Proposition 4.1, we start to prove Theorem 1.2.

Proof of Theorem 1.2. By Theorem 1.1, we know that problem (2.5a)-(2.5b) has a local solution $(w, v)$ such that $w \in C\left([0, T], H^{3}\left(S_{0}\right)\right) \cap C^{1}\left([0, T], H^{2}\left(S_{0}\right)\right)$ and $v \in C\left([0, T], H_{0}^{1}\left(S_{0}\right) \cap\right.$ $\left.H^{3}\left(S_{0}\right)\right) \cap C^{1}\left([0, T], H_{0}^{1}\left(S_{0}\right)\right) \cap L^{2}\left([0, T], H^{4}\left(S_{0}\right)\right)$. From Proposition 4.1, the uniform energy estimates for $t^{\prime} \geq 1$ are obtained when the initial norm $\left\|\left(w_{0}, v_{0}\right)\right\|_{H^{3}} \leq \varepsilon_{0}$ is small. Therefore, it follows the continuity argument that problem (2.5a)-(2.5b) has a global small solution and further (1.3a)-(1.3c) in Theorem 1.2 hold. 


\section{Acknowledgements}

The authors were supported by the NSFC (No. 11571177 and No. 11731007) and a Project Funded by the Priority Academic Program Development of Jiangsu Higher Education Institutions.

\section{References}

[1] Cho Yonggeun, Choe Hi Jun and Kim Hyunseok, Unique solvability of the initial boundary value problems for compressible viscous fluids, J. Math. Pures Appl., 83 (2004), 243-275.

[2] Cho Yonggeun and Kim Hyunseok, On classical solutions of the compressible Navier-Stokes equations with nonnegative initial densities, Manuscript Math., 120 (2006), 91-129.

[3] R. Danchin, Global existence in critical spaces for compressible Navier-Stokes equations, Invent. Math., 141 (2000), 579-614.

[4] E. Feireisl, Dynamics of Viscous Compressible Fluids, Oxford University Press, New York, 2004.

[5] E. Feireisl, O. Kreml, S. Necasova, J. Neustupa and J. Stebel, Weak solutions to the barotropic Navier-Stokes system with slip boundary conditions in time dependent domains, J. Differential Equations, 254(1) (2013), 125-140.

[6] D. Hoff, Discontinuous solution of the Navier-Stokes equations for multi-dimensional heatconducting fluids, Arch. Ration. Mech. Anal., 193 (1997), 303-354.

[7] Huang Xiangdi, Li Jing and Xin Zhouping, Global well-posedness of classical solutions with large oscillations and vacuum to the three-dimensional isentropic compressible NavierStokes equations, Commun. Pure Appl. Math., 65 (2012), 549-585.

[8] Jiang Song, Global spherically symmetric solutions to the equations of a viscous polytropic ideal gas in an exterior domain, Commun. Math. Phys., 178 (1996), 339-374.

[9] Jiang Song and Zhang Ping, Global spherically symmetric solutions of the compressible isentropic Navier-Stokes equations, Commun. Math. Phys., 215 (2001), 559-581.

[10] Y. Kagei and S. Kawashima, Stability of planar stationary solutions to the compressible Navier-Stokes equation on the half space, Commun. Math. Phys., 266 (2006), 401-430.

[11] P. L. Lions, Mathematical Topics in Fluid Mechanics, Vol. 2. Compressible Models, Oxford University Press, New York, 1998.

[12] A. Matsumura and T. Nishida, The initial value problem for the equations of motion of viscous and heat-conductive gases, J. Math. Kyoto Univ., 20(1) (1980), 67-104.

[13] A. Matsumura and T. Nishida, Initial boundary value problems for the equations of motion of compressible viscous and heat conductive fluids, Commun. Math. Phys., 89 (1983), 445464 .

[14] J. Nash, Le probleme de Cauchy pour les equations differentielles d'un fluide general, Bull. Soc. Math. France, 90 (1962), 487-497.

[15] D. Serre, Solutions faibles globales des équations de Navier-Stokes pour un fluide compressible, C. R. Acad. Sci. Paris Sér. I Math., 303 (1986), 639-642.

[16] V. A. Vaigant and A. V. Kazhikhov, On the existence of global solutions of two-dimensional Navier-Stokes equations of a compressible viscous fluid, (Russian) Sibirsk. Mat. Zh., 36 (1995), 1283-1316, ii; translation in Siberian Math. J., 36 (1995), 1108-1141.

[17] Xu Gang and Yin Huicheng, The global existence and large time behavior of smooth compressible fluid in an infinitely expanding ball, I: 3D Euler equations, Phys. Scr., 93(10) (2018), 
105001.

[18] Yang Tong, Yao Zhengan and Zhu Changjiang, Compressible Navier-Stokes equations with density dependent viscosity and vacuum, Commun. Partial Differential Equations, 26(5-6) (2001), 965-981.

[19] Yin Huicheng and Zhang Lin, The global existence and large time behavior of smooth compressible fluid in an infinitely expanding ball, II: 3D Navier-Stokes equations, Discrete and Continuous Dynamical System-A, 38(3) (2018), 1063-1102.

[20] Yin Huicheng and Zhao Wenbin, The global existence and large time behavior of smooth compressible fluid in an infinitely expanding ball, III: 3D Boltzmann equations, J. Differential Equations, 264 (2018), 30-81. 